Aus der Abteilung Neurologie

(Prof. Dr. med. M. Bähr)

im Zentrum Neurologische Medizin

der Medizinischen Fakultät der Universität Göttingen

\title{
Genetische Polymorphismen und Progressionsgeschwindigkeit der Alzheimer-Demenz
}

\author{
INAUGURAL-DISSERTATION \\ zur Erlangung des Doktorgrades
}

der Medizinischen Fakultät der

Georg-August-Universität zu Göttingen

vorgelegt von

MARTIN WOLFF

aus

Braunschweig

Göttingen 2012 
Dekan: Prof. Dr. rer. nat. H. K. Kroemer

I. Berichterstatterin: Prof. Dr. med. I. Zerr II. Berichterstatter/-in: Prof. Dr. med. T. G. Schulze III. Berichterstatter/-in: Prof. Dr. H. Bickeböller Tag der mündlichen Prüfung: 22.10.2013 


\section{Inhaltsverzeichnis}

Abkürzungsverzeichnis $\quad$ IV

Abbildungsverzeichnis $\quad$ VII

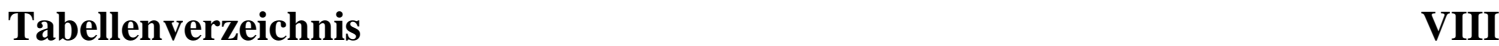

1. Einleitung 1

1.1. Einführung in das Thema Demenzen 1

1.2. Die Demenz vom Alzheimer-Typ (AD) 2

1.2.1. Definition und Übersicht 2

1.2.2. Geschichte 2

1.2.3. Epidemiologie und Risikofaktoren 3

1.2.4. Klinisches Bild 4

1.2.4.1. Kognitive Symptome 5

1.2.4.2. Psychiatrische Begleitsymptome 5

1.2.4.3. Neurologische Symptome $\quad 5$

1.2.5. Diagnostik 6

1.2.5.1. Klinische Kriterien 6

1.2.5.2. Bildgebung 6

$\begin{array}{ll}\text { 1.2.5.3. Biomarker im Liquor } & 7\end{array}$

1.2.6. Pathogenese 8

1.2.6.1. $\beta$-Amyloid 8

$\begin{array}{lll}\text { 1.2.6.2. Tau-Protein } & 10\end{array}$

1.2.6.3. Veränderungen der Neurotransmitter 10

1.2.7. Therapie 11

$\begin{array}{lll}\text { 1.2.7.1. Cholinesterasehemmer } & 11\end{array}$

1.2.7.2. Memantine 11

$\begin{array}{ll}\text { 1.2.7.3. Präventive Maßnahmen } & 12\end{array}$

1.3. Genetik der Alzheimer-Demenz 12

1.3.1. Amyloid-Precursor-Protein (APP) 12

$\begin{array}{ll}\text { 1.3.2. Präsenilin (PSEN) } 1 \text { und } 2 & 13\end{array}$

1.3.3. Apolipoprotein E (ApoE) 13

$\begin{array}{ll}\text { 1.3.4. Andere Suszeptibilitätsgene } & 14\end{array}$

1.4. Die „rapid-progressive Alzheimer-Demenz“ (rpAD) 15

1.4.1. Überblick und Definition $\quad 15$

$\begin{array}{ll}\text { 1.4.2. Klinik } & 16\end{array}$

1.4.3. Komorbidität und Risikofaktoren 16

$\begin{array}{ll}\text { 1.4.4. Biomarker } & 17\end{array}$

$\begin{array}{ll}\text { 1.4.5. Genetik } & 17\end{array}$

$\begin{array}{lr}\text { 1.5. Fragestellung } & 19\end{array}$ 
2. Patienten und Methoden $\quad 20$

2.1. Studiendesign und Patientenrekrutierung 20

2.1.1. Hintergrund 20

2.1.2. Die rpAD-Studie 20

2.1.3. Patientenrekrutierung 21

2.1.4. Aufklärung und Einwilligung 22

2.1.5. Einschlusskriterien 22

2.1.6. Ausschlusskriterien 22

2.2. Erhobene und gemessene Parameter 23

2.2.1. Anamnese und Untersuchung 23

2.2.2. Neuropsychologische Tests 23

2.2.2.1. Mini-Mental-Status-Test (MMST) 23

2.2.2.2. CERAD-Testbatterie 24

2.2.3. Liquordiagnostik 25

2.2.3.1. Gesamt-Tau 25

2.2.3.2. Phospho-Tau 25

2.2.3.3. $\beta$-Amyloid 25

2.2.3.4. Protein 14-3-3 26

2.2.4. Genetische Marker 26

2.3. Durchführung 28

2.4. Erfassung und Auswertung der Daten 29

2.4.1. Dateneingabe und -verarbeitung 29

2.4.2. Statistische Analysen 29

3. Ergebnisse 31

3.1. Beschreibung des Patientenkollektivs 31

3.1.1. Soziodemographische Daten 31

3.1.1.1. Alter und Geschlecht $\quad 31$

3.1.1.2. Krankheitsbeginn und Krankheitsdauer 32

3.1.2. Kognitiver Status 33

3.1.2.1. MMST bei Erst- und Zweituntersuchung 33

3.1.2.2. MMST-Progression $\quad 35$

3.2. Genetische Polymorphismen 38

3.2.1. Genotyp- und Allelverteilung im Gesamtkollektiv 38

$\begin{array}{lll}\text { 3.2.1.1. PICALM } & 41\end{array}$

$\begin{array}{lll}\text { 3.2.1.2. } & \text { BIN1 } & 42\end{array}$

3.2.1.3. TNK1 44

3.2.2. Verteilung der Allelträger bei Frauen und Männern 46

3.2.2.1. BIN1 48

3.2.2.2. GWA14q32.13 49

3.2.2.3. ACE 50

3.2.2.4. LDLR 51 
3.2.3. Allelverteilung bei Patienten mit EOAD und LOAD 52

$\begin{array}{lll}\text { 3.2.3.1. } & \text { PICALM } & 55\end{array}$

$\begin{array}{lll}\text { 3.2.3.2. CST3 } & 56\end{array}$

4. Diskussion $\quad 58$

4.1. Studiendesign und Methodik 58

4.2. Soziodemographische Daten und kognitiver Status 60

4.2.1. Alter und Geschlecht $\quad 60$

$\begin{array}{ll}\text { 4.2.2. Krankheitsdauer } & 61\end{array}$

4.2.3. MMST bei Erst- und Zweituntersuchung 62

4.2.4. MMST-Progression $\quad 62$

4.3. Genetische Polymorphismen 63

4.3.1. PICALM 63

4.3.2. BIN1 65

4.3.3. TNK1 67

4.3.4. Weitere Polymorphismen $\quad 68$

$\begin{array}{lll}\text { 4.3.4.1. } & \text { GWA14q32.13 } & 68\end{array}$

$\begin{array}{lll}\text { 4.3.4.2. } & \text { ACE } & 69\end{array}$

4.3.4.3. LDLR $\quad 69$

$\begin{array}{lll}\text { 4.3.4.4. } & \text { CST3 } & 70\end{array}$

4.3.4.5. ApoE, CLU, IL8 und EXOC3L2 71

$\begin{array}{ll}\text { 4.4. Schlussfolgerung } & 77\end{array}$

$\begin{array}{ll}\text { 5. Zusammenfassung } & 80\end{array}$

$\begin{array}{lr}\text { 6. Anhang } & 81\end{array}$

6.1. Revidierte NINCDS-ADRDA-Kriterien nach Dubois $\quad 81$

6.2. Aufklärungsbogen (Version für Angehörige und Betreuer) 82

6.3. Einwilligungsbogen (Version für Angehörige und Betreuer) 84

6.4. Standardisierter Fragebogen 86

7. Literaturverzeichnis 92

$\begin{array}{ll}\text { 8. Publikationen } & 113\end{array}$ 


\section{Abkürzungsverzeichnis:}

A $\beta: \quad \quad \beta$-Amyloid

Abb.:

Abbildung

AD:

Alzheimer-Demenz, Demenz vom Alzheimer-Typ

ADAS-Cog:

Alzheimer's Disease Assessment Scale - Cognitive Subscale

ADCS-ADL:

Alzheimer's Disease Cooperative Study-Activities of Daily Living

Scale

AD \& FTDMDB: $\quad$ Alzheimer Disease and Frontotemporal Dementia Mutation Database

ADL: Activities of Daily Living

ADRDA:

Alzheimer's Disease and Related Disorders Association

ANOVA:

Analysis of Variance

APA:

American Psychiatric Association

Apo E:

Apolipoprotein E

APP:

Amyloid-Precursor-Protein

B-ADL:

Basic Activities of Daily Living

BCRS:

Brief Cognitive Rating Scale

$\mathrm{BD}(\mathrm{R}) \mathrm{S}$ :

Blessed Dementia (Rating) Scale

BRD:

Bundesrepublik Deutschland

BS:

Balance Scale

CAMCOG:

Cambridge Cognitive Examination

CDR:

Clinical Dementia Rating (Scale)

CERAD:

Consortium for the Establishment of a Registry for Alzheimer's Disease

CIBIC-Plus:

Clinician's Interview-Based Impression of Change Plus Caregiver Input

CT:

Computertomographie

DAD:

Disability Assessment in Dementia

DNS:

Desoxyribonukleinsäure

DRS:

Dementia Rating Scale

DS-CAMDEX: $\quad$ Dementia Scale of Cambridge Mental Disorders of the Elderly Examination

DSM-IIIR: $\quad$ Diagnostic and Statistical Manual of Mental Disorders Third Edition (Revision)

DSM-IV:

Diagnostic and Statistical Manual of Mental Disorders Fourth Edition 
EEG: $\quad$ Elektroenzephalographie

ELISA: $\quad$ Enzyme-linked immunosorbent assay

EOAD: $\quad$ Early-Onset Alzheimer's Disease: Alzheimer-Demenz mit frühem Erkrankungsbeginn $\leq 65$. Lebensjahr

et al.: $\quad$ et alii

EU: $\quad$ Erstuntersuchung

FDG: $\quad{ }^{18}$ F-Fluor-Desoxyglucose

FTD: $\quad$ Fronto-temporale Demenz

GDS: $\quad$ Global Deterioration Scale

GSK 3: $\quad$ Glycogen-Synthase-Kinase 3

GWA(S): $\quad$ Genome-wide association (studies)

HDSR: Hasegawa's Dementia Screening-Revised

HWG: Hardy-Weinberg-Gleichgewicht

I-ADL: $\quad$ Instrumental Activities of Daily Living

ICD-10: $\quad$ International Statistical Classification of Diseases and Related Health Problems 10th Revision

IMC-BDS: $\quad$ Information-Memory-Concentration subtest of the Blessed Dementia Scale

IQR: Interquartile range (Interquartilsabstand)

kDa: $\quad$ Kilodalton

KHK: $\quad$ Koronare Herzkrankheit

LBD: $\quad$ Lewy-Body-Demenz

LOAD: $\quad$ Late-Onset Alzheimer's Disease: Alzheimer-Demenz mit spätem Erkrankungsbeginn > 65. Lebensjahr

MCI: $\quad$ Mild cognitive impairment

MMST: Mini-Mental-Status-Test

MRT: $\quad$ Magnetresonanztomographie

MTL: medialer Temporallappen

MW: $\quad$ Mittelwert $(=\bar{x})$

$\mathrm{n}: \quad$ Anzahl

NFT: $\quad$ Neurofibrilläre Tangles

NICE-SCIE: $\quad$ The National Institute for Health and Clinical Excellence (NICE) and the Social Care Institute for Excellence (SCIE) 
NINCDS:

NPI:

NRZ:

o.g.:

OR:

PASW:

PET:

PGDRS:

PHF:

PIB:

PSEN:

PSMS:

REAL.FR:

RLS:

rpAD:

S.:

SD:

SP:

SPECT:

SPSS:

tMMST:

u.:

UPDRS III:

VD:

vs.:

WHO:

$\tilde{x}:$

$\bar{x}:$

ZU:
National Institute of Neurological and Communicative Disorders and Stroke

Neuropsychiatric Inventory

Nationales Referenzzentrum für die Surveillance Transmissibler Spongiformer Enzephalopathien der Universitätsmedizin Göttingen oben genannt

Odds ratio

Predictive Analysis Software

Positronenemissionstomographie

Psychogeriatric Dependency Rating Scale

Paired helical filaments

Pittsburgh Compound B

Präsenilin

Physical Self-Maintenance Scale

Réseau sur la Maladie d'Alzheimer Français (Multizentrische prospektive Alzheimer-Studie in Frankreich)

Reaction Level Scale

rapid-progressive Alzheimer-Demenz

Seite

Standard deviation (Standardabweichung)

Senile Plaques

Single-Photon-Emissions-Computertomographie

Statistical Package for the Social Sciences

telefonischer Mini-Mental-Status-Test

und

Unified Parkinson's Disease Rating Scale, Section III

Vaskuläre Demenz

versus

World Health Organization (Weltgesundheitsorganisation)

Median

Mittelwert

Zweituntersuchung 


\section{Abbildungsverzeichnis}

Abb. 1: Häufigkeiten der Hauptformen der Demenzen (modifiziert nach: 1

Mahlberg und Gutzmann 2005, S. A2034)

Abb. 2: $\quad$ Die Amyloid-Kaskadenhypothese (modifiziert nach: Selkoe 2008, S. 253)

Abb. 3: $\quad$ Aufbau der rpAD-Studie 21

Abb. 4: Geschlechtsspezifischer Altersunterschied im Patientenkollektiv 32

Abb. 5: MMST bei der Erst-und Zweituntersuchung 34

Abb. 6: Geschlechtsspezifische Unterschiede im MMST bei 34

Erstuntersuchung

Abb. 7: $\quad$ Geschlechtsspezifische Unterschiede im MMST bei 35

Zweituntersuchung

Abb. 8: $\quad$ MMST-Progression der PICALM-Genotypen 41

Abb. 9: $\quad$ PICALM: MMST-Progression von C-Trägern und 42

Nicht-C-Trägern

Abb. 10: BIN1-Genotypen und Krankheitsverlauf („slow“" vs. „rapid“) 43

Abb. 11: MMST-Progression der BIN1-Genotypen 43

Abb. 12: $\quad$ BIN1: MMST-Progression von A-Trägern und Nicht-A-Trägern 44

Abb. 13: $\quad$ MMST-Progression der TNK1-Genotypen 45

Abb. 14: TNK1: MMST-Progression der T-Träger und Nicht-T-Träger 45

Abb. 15: BIN1: MMST-Progression von A-Trägern und Nicht-A-Trägern 49 bei Frauen

Abb. 16: GWA14q32.12: MMST-Progression von T-Trägern und $\quad 50$ Nicht-T-Trägern bei Männern

Abb. 17: ACE: MMST-Progression von C-Trägern und Nicht-C-Trägern 51 bei Frauen

Abb. 18: $\quad$ LDLR: MMST-Progression von G-Trägern und 52

Nicht-G-Trägern bei Frauen

Abb. 19: PICALM: MMST-Progression von C-Trägern und Nicht-C- 56

Trägern bei Patienten mit spätem Krankheitsbeginn

Abb. 20: CST3: MMST-Progression von T-Trägern und Nicht-T-Trägern bei Patienten mit frühem Krankheitsbeginn 


\section{Tabellenverzeichnis}

Tabelle 1: ICD-10-Kriterien für das Vorliegen einer Demenz (modifiziert 2 nach: Schaub und Freyberger 2005, S. 60)

Tabelle 2: $\quad$ Risikogene und Odds Ratio, Stand Februar 2010

(Bertram et al. 2007)

Tabelle 3: Definitionen und Häufigkeiten von rpAD in Studien 16

Tabelle 4: $\quad$ Einfluss genetischer Polymorphismen auf den Verlauf der 18 $\mathrm{AD}$ (Beispiele)

Tabelle 5: Untertests der CERAD-Testbatterie 24

Tabelle 6: Primer der untersuchten Polymorphismen (Angaben jeweils als 27 $5^{6}$-Sequenz-3')

Tabelle 7: Hardy-Weinberg-Gleichgewicht der untersuchten 28 Polymorphismen

Tabelle 8: $\quad$ Alter der Patienten 31

Tabelle 9: Krankheitsbeginn der Patienten 32

Tabelle 10: Krankheitsdauer der Studienteilnehmer (in Monaten) 33

Tabelle 11: MMST-Punktzahlen bei Erst- (EU) und Zweituntersuchung (ZU) 33

Tabelle 12: MMST-Progression in Abhängigkeit von Geschlecht und 36 Krankheitsbeginn

Tabelle 13: Korrelation zwischen MMST-Progression und MMST, Alter, 36 Ausbildung und Krankheitsdauer bei Erstuntersuchung

Tabelle 14: Krankheitsverlauf in Abhängigkeit von Geschlecht und Krankheitsbeginn

Tabelle 15: Krankheitsverlauf in Abhängigkeit von MMST, Alter, Ausbildung und Krankheitsdauer bei Erstuntersuchung

Tabelle 16: Untersuchte Polymorphismen: Genotyp- und Allelverteilung mit jeweiliger Progression

Tabelle 17: Untersuchte Polymorphismen nach Geschlecht stratifiziert:

Allelverteilung und jeweilige Progression

Tabelle 18: Untersuchte Polymorphismen nach Krankheitsbeginn stratifiziert: Allelverteilung und jeweilige Progression 
Tabelle 19: Übersicht relevanter ApoE-Studien Teil 1: Schnellere

Krankheitsprogression der AD bei Anwesenheit von ApoE $\varepsilon 4$

(modifiziert und erweitert nach Kleiman et al. 2006, S. 78)

Tabelle 20: Übersicht relevanter ApoE-Studien Teil 2: Langsamere

Krankheitsprogression der AD bei Anwesenheit von ApoE $\varepsilon 4$

(modifiziert und erweitert nach Kleiman et al. 2006, S. 78)

Tabelle 21: Übersicht relevanter ApoE-Studien Teil 3: Kein Einfluss von

ApoE $\varepsilon 4$ auf die Krankheitsprogression der AD (modifiziert und erweitert nach Kleiman et al. 2006, S. 78)

Tabelle 22: Zusammenfassung der untersuchten Polymorphismen

Tabelle 23: Revidierte NINCDS-ADRDA-Kriterien (modifiziert nach

Dubois et al. 2007, S. 738) 


\section{Einleitung}

\subsection{Einführung in das Thema Demenzen}

Der Begriff Demenz bezeichnet laut WHO ein klinisches Syndrom, welches durch eine chronische oder progrediente Störung der Gedächtnis- und Denkleistungen charakterisiert wird. Begleitet werden können diese Einschränkungen von Veränderungen der emotionalen Kontrolle, des Sozialverhaltens oder der Motivation. Die Leistungseinbußen müssen mindestens sechs Monate andauern (WHO et al. 2011a). Ein demenzielles Syndrom ist im Gegensatz zu angeborenen Minderbegabungen immer erworben und kann durch eine Vielzahl von Krankheiten verursacht werden. Die häufigste Ursache ist die Demenz vom AlzheimerTyp, gefolgt von der vaskulären Demenz und der Lewy-Body-Erkrankung (Mahlberg und Gutzmann 2005, siehe Abb. 1). Die Diagnosestellung einer Demenz erfolgt anhand von ICD10- (WHO et al. 2011a, siehe Tabelle 1) oder DSM-IV-Kriterien (APA 2000).

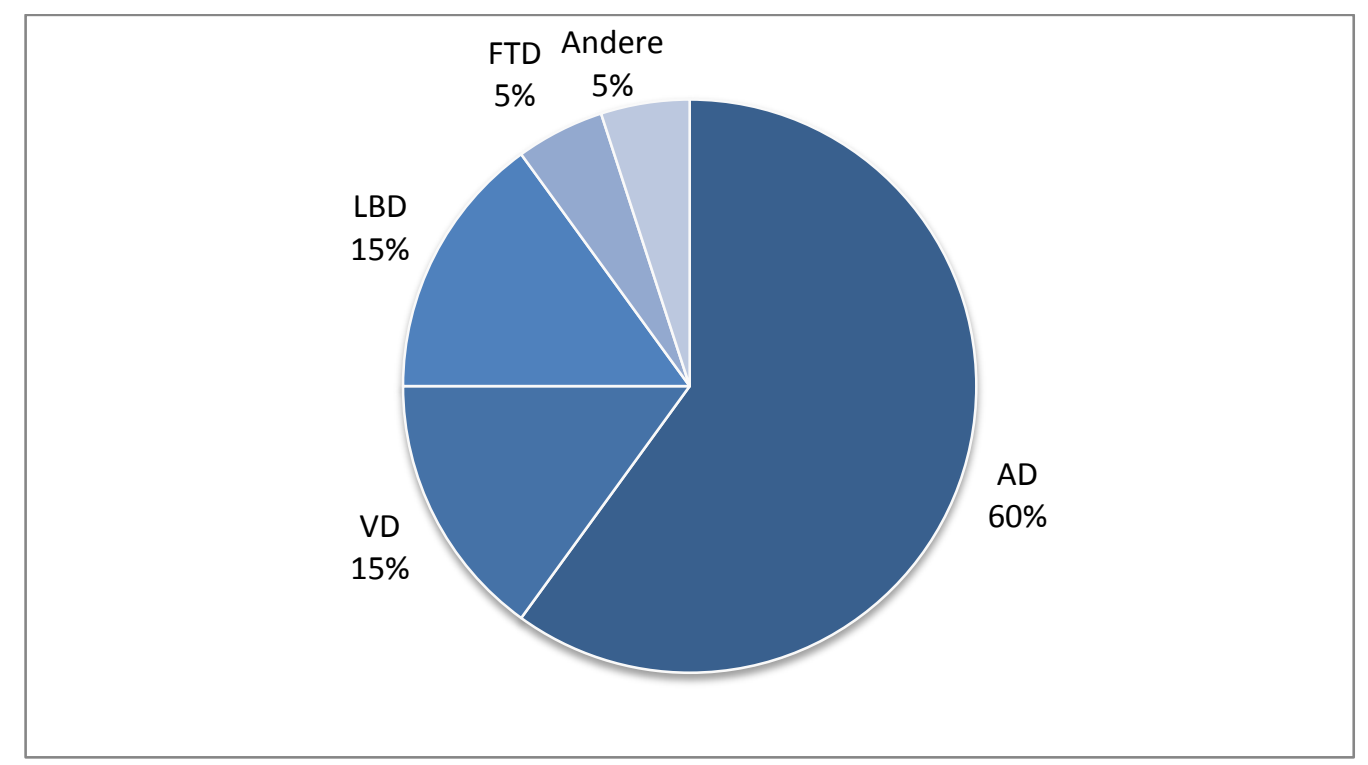

AD: Demenz vom Alzheimer-Typ, VD: Vaskuläre Demenz, LBD: Lewy-Body-Demenz, FTD: Fronto-temporale Demenz, Andere: Andere Demenzformen

Abb. 1: Häufigkeiten der Hauptformen der Demenzen (modifiziert nach: Mahlberg und Gutzmann 2005, S. A2034) 
Tabelle 1: ICD-10-Kriterien für das Vorliegen einer Demenz (modifiziert nach: Schaub und Freyberger 2005, S. 60):

\begin{tabular}{ll}
\hline G1 1. Abnahme des Gedächtnisses \\
2. Abnahme anderer kognitiver Fähigkeiten, charakterisiert durch eine \\
Verminderung der Urteilsfähigkeit und des Denkvermögens \\
(Beeinträchtigung der Aktivitäten des täglichen Lebens durch die kognitiven \\
Defizite) \\
G2: Fehlen einer Bewusstseinstrübung oder deliranter Episoden \\
G3: Verminderung der Affektkontrolle, des Antriebs oder des Sozialverhaltens \\
G4: Die Störungen von G1 sollten mindestens 6 Monate vorhanden sein
\end{tabular}

\subsection{Die Demenz vom Alzheimer-Typ (AD)}

\subsubsection{Definition und Übersicht}

Die $\mathrm{AD}$ ist eine typischerweise langsam progrediente, neurodegenerative Erkrankung, deren Überlebenszeit ca. 3 - 9 Jahre beträgt (Goldberg 2007; Querfurth und LaFerla 2010). Die Erkrankung wird durch eine zunehmende Störung des Gedächtnisses sowie weiterer kognitiver Funktionen charakterisiert (Hodges 2006), die durch Verhaltensauffälligkeiten sowie Einschränkungen der Alltagsaktivitäten begleitet werden (Grossberg 2002; Potkin 2002). Aus neuropathologischer Sicht wird die Erkrankung durch senile Plaques, neurofibrilläre Tangles und einen Verlust von Nervenzellen und Synapsen charakterisiert (Blennow et al. 2006). Anhand des Zeitpunktes des Krankheitsbeginns kann die AD in eine früh auftretende Variante vor dem 65. Lebensjahr (Early-Onset-AD, EOAD) und eine nach dem 65. Lebensjahr auftretende Late-Onset-Form ( $L O A D)$ eingeteilt werden. Des Weiteren unterscheidet man zwischen familiären und sporadischen Fällen, wobei letztere den weitaus größeren Teil ausmachen.

\subsubsection{Geschichte}

Der Name der Erkrankung geht auf den Psychiater Alois Alzheimer zurück (1864-1915). Dieser beschrieb in seiner 1907 verfassten Abhandlung „Über eine eigenartige Erkrankung der Hirnrinde" sowohl die typische Klinik als auch die charakteristischen 
neuropathologischen Befunde (Alzheimer 1907). Die Benennung der Krankheit nach ihrem Entdecker ist Emil Kraepelins erstmaliger Erwähnung in dessen Lehrbuch der Psychiatrie von 1910 geschuldet (Hodges 2006).

\subsubsection{Epidemiologie und Risikofaktoren}

Die AD ist mit circa 50-60\% die häufigste Form aller Demenzerkrankungen (Blennow et al. 2006). In Deutschland ist derzeit mit etwa einer Million Demenzkranken zu rechnen, die Inzidenz wird auf ungefähr 244000 pro Jahr beziffert (Ziegler und Doblhammer 2009). Gab es laut Schätzungen 2001 weltweit circa 24 Millionen Erkrankte, so wird erwartet, dass sich diese Zahl aufgrund der steigenden Lebenserwartung alle 20 Jahre verdoppelt (Ferri et al. 2005). Neben dem für die Patienten und Angehörigen tragischen Verlauf ist die Erkrankung auch volkswirtschaftlich von größter Bedeutung. Für die BRD betrugen nach Rechnungen des statistischen Bundesamtes die Kosten für Demenzerkrankungen im Jahre 2008 ca. 9,4 Milliarden Euro (www.destatis.de 2010). Die weltweiten Ausgaben wurden für das Jahr 2009 auf 422 Milliarden Dollar geschätzt (Wimo et al. 2010).

Der stärkste Risikofaktor für die $\mathrm{AD}$ ist das Alter. Die Prävalenz der Demenzen steigt von unter $1 \%$ in der Altersgruppe von 60 bis 64 Jahren nahezu exponentiell mit dem Alter an und liegt in der westlichen Welt bei Menschen ab 85 Jahren bei 24-33 \% (Ferri et al. 2005). Aufgrund der höheren Lebenserwartung sind Frauen deutlich häufiger von Demenzerkrankungen betroffen (Lobo et al. 2000).

Ebenso spielen genetische Einflüsse eine sehr große Rolle in der Ätiologie der AlzheimerDemenz. Bei der seltenen familiären Form handelt es sich um eine Gruppe von autosomaldominanten Erkrankungen, die durch einen sehr frühen Beginn zwischen dem 30. und 50. Lebensjahr gekennzeichnet sind. Ursächliche Mutationen wurden im APP-Gen auf Chromosom 21 (Goate et al. 1991), im PSEN-1-Gen auf Chromosom 14 (Sherrington et al. 1995) sowie im PSEN-2-Gen auf Chromosom 1 (Levy-Lahad et al. 1995) gefunden. Bei der weitaus häufigeren nicht-familiären $\mathrm{AD}$ wurde 1993 das Apo-ع4-Allel als bedeutender Risikofaktor beschrieben (Corder et al. 1993; Poirier et al. 1993). In der Literatur wird derzeit eine Vielzahl weiterer Suszeptibilitäts-Gene diskutiert (Bertram et al. 2007). Patienten mit Trisomie 21 haben darüberhinaus ebenfalls ein erhöhtes Risiko, an einer AD zu erkranken (Holland et al. 1998). Für eine ausführliche Übersicht der genetischen Aspekte der AD wird auf Kapitel 1.3 verwiesen. 
Kardio- und zerebrovaskuläre Komorbiditäten (Nikotinabusus, KHK, arterielle Hypertonie, Hyperlipidämie, Homocysteinämie, Adipositas, Diabetes) scheinen durch verschiedene Mechanismen sowohl die Entstehung als auch den Verlauf der AD beeinflussen zu können (Mayeux 2008; Barnes und Yaffe 2011; Etgen et al. 2011). Mehrfach wurde auch ein möglicher Zusammenhang zwischen Schädel-Hirn-Traumen und der Entwicklung einer AD beschrieben (Jellinger 2004).

Bestimmten psychosozialen Faktoren (hohes Ausbildungsniveau, regelmäßige geistige und körperliche Betätigung, ausgeprägtes soziales Netzwerk) wird eine protektive Rolle zugeschrieben, da sie den Erkrankungsbeginn möglicherweise hinauszögern (Qiu et al. 2009; Barnes und Yaffe 2011). Einige Ernährungsstudien lassen vermuten, dass eine hochdosierte Einnahme von Vitamin-B12, Folsäure, Antioxidanzien (Vitamin A, C, E) sowie von Omega3-Fettsäuren und maßvoller Alkoholkonsum mit einem niedrigeren Risiko einer $\mathrm{AD}$ einhergehen. Die Ergebnisse sind jedoch widersprüchlich (Luchsinger und Mayeux 2004; Etgen et al. 2011). Der mögliche protektive Effekt anti-inflammatorischer sowie östrogenhaltiger Agenzien ist ebenfalls Gegenstand epidemiologischer Studien (Mayeux 2008).

\subsubsection{Klinisches Bild}

Die AD kann anhand des Mini-Mental-Status-Tests (MMST, Folstein et al. 1975) in ein frühes (MMST 20-26 Punkte), ein mittleres (MMST 10-19 Punkte) und ein spätes Stadium (MMST < 10 Punkte) eingeteilt werden (NICE-SCIE 2007). Im Vordergrund stehen zunächst kognitive Defizite sowie begleitende psychiatrische Störungen, während körperlichneurologische Symptome erst spät im Verlauf auftreten (Schmidtke und Hüll 2005).

Der klinischen Diagnose kann bereits einige Jahre zuvor eine Phase ohne manifeste Demenz vorangehen (Mild Cognitive Impairment, MCI). Dieses zeichnet sich durch eine Störung des Gedächtnisses oder anderer kognitiver Fähigkeiten aus, die über das für Alter und Ausbildung zu erwartende Maß hinausgehen, jedoch keine Beeinträchtigung der alltäglichen Fähigkeiten hervorrufen (Gauthier et al. 2006). Man unterscheidet ein amnestisches MCI (Gedächtnisdefizit) von einem nicht-amnestischen MCI (andere kognitive Defizite). Bei Patienten mit amnestischem MCI beträgt die Konversionsrate 10-15\% pro Jahr bezüglich des Übergangs in eine manifeste AD (Petersen und Negash 2008). 


\subsubsection{Kognitive Symptome}

Kognitive Defizite stellen bei der AD die Kernsymptomatik dar, wobei sich typischerweise die Gedächtnisstörung als Haupt- und Frühsymptom präsentiert (Corey-Bloom 2002). In erster Linie ist hierbei das episodische Neugedächtnis gestört, das Arbeits- und das Langzeitgedächtnis verschlechtern sich meist erst im Krankheitsverlauf (Hodges 2006). Schreitet die Erkrankung fort, treten Störungen des semantischen Gedächtnisses sowie Beeinträchtigungen der Aufmerksamkeit, der exekutiven Funktionen und des räumlichvisuellen Denkens hinzu (Hodges 2006). Sprachstörungen machen sich zu Beginn der Erkrankung meist durch Wortfindungsstörungen und Benennungsschwierigkeiten bemerkbar, im Verlauf treten neben einer inhaltsarmen und floskelhaften Sprache jedoch auch Störungen des Sprachverständnisses und der Sprachproduktion auf (Honig und Mayeux 2001). Meist in fortgeschrittenen Stadien lassen sich eine Apraxie sowie eine visuelle Agnosie beobachten (Schmidtke und Hüll 2005). Die kognitiven Einschränkungen beeinflussen zunehmend die Urteils- und Planungsfähigkeit sowie die alltäglichen Aktivitäten der Patienten (Förstl und Kurz 1999).

\subsubsection{Psychiatrische Begleitsymptome}

Verhaltensveränderungen und psychische Symptome können während aller Stadien der Erkrankung zu finden sein. Bereits in der präklinischen Phase einer AD können gelegentlich z.B. depressive Veränderungen oder ein sozialer Rückzug beobachtet werden (Jost und Grossberg 1995). Im frühen Verlauf der Erkrankung scheinen besonders Apathie, Depression und Angst aufzutreten, während in mittleren bis späten Stadien auch Wahnvorstellungen, Halluzinationen und Agitation beobachtet werden können (McKeith und Cummings 2005). Als weitere Verhaltensauffälligkeiten werden Wandersucht, Esssucht, Hyperoralität, Urininkontinenz, Hypersexualität und Störungen des Schlaf-Wach-Rhythmus beschrieben (Burns et al. 1990; Stopa et al. 1999).

\subsubsection{Neurologische Symptome}

Spezielle neurologische Symptome treten meist erst in sehr späten Erkrankungsstadien auf. $\mathrm{Zu}$ den im fortgeschrittenen Krankheitsverlauf am häufigsten berichteten neurologischen Symptomen gehören Rigor, Myoklonien und epileptische Anfälle (Förstl et al. 1992). Weiterhin können auch frontale Enthemmungszeichen beobachtet werden (Burns et al. 1991). 


\subsubsection{Diagnostik}

\subsubsection{Klinische Kriterien}

Die in deutschen Kliniken verwendete ICD-10-Klassifikation setzt für die Diagnose einer AD zunächst das Vorliegen der allgemeinen Demenzkriterien voraus (siehe Kapitel 1.1, Tabelle 1, S. 2). Weiterhin dürfen weder Anamnese noch Untersuchung Hinweise auf eine andere Demenzursache, eine Systemerkrankung oder einen Alkohol- oder Substanzmissbrauch geben (WHO et al. 2011b). Es handelt sich damit überwiegend um eine Ausschlussdiagnose.

Die wissenschaftlichen NINCDS-ADRDA-Kriterien (McKhann et al. 1984) unterscheiden eine definitive, eine wahrscheinliche sowie eine mögliche AD. Das Vorhandensein einer anderen demenzverursachenden Erkrankung ist nicht grundsätzlich ein Ausschlusskriterium, sondern kann mit einer möglichen $\mathrm{AD}$ vereinbar sein, solange die zusätzliche Erkrankung nicht als die wesentliche Ursache der Demenz angesehen wird. Eine definitive AD kann nur bei gemeinsamem Vorliegen der klinischen Kriterien einer wahrscheinlichen AD und zusätzlicher histopathologischer Sicherung diagnostiziert werden.

Aufgrund mangelnder Sensitivität und Spezifität der o.g. Kriterien sowie Fortschritten in der Erforschung verlässlicher Biomarker wurde eine revidierte Fassung der NINCDS-ADRDAKriterien vorgeschlagen (Dubois et al. 2007, siehe Anhang, Tabelle 23, S. 81).

\subsubsection{Bildgebung}

\section{$\underline{\mathrm{CT} / \mathrm{MRT}}$}

Die AD zeichnet sich durch eine globale Hirnatrophie aus, wobei die Degeneration des medialen Temporallappens (MTL) einschließlich Hippokampus und entorhinalem Kortex zu den frühesten Veränderungen gehört (Braak et al. 1999). Besonders in der Darstellung der MTL-Strukturen ist die MRT-Technik der CT überlegen und stellt daher das bevorzugte Verfahren dar (Lehericy et al. 2007). Die Atrophie im MTL korreliert dabei mit der klinischen Progression und kann bei Patienten mit MCI eine Konversion zur AD vorhersagen (Jack et al. 1999; Jack et al. 2000; Rusinek et al. 2004).

\section{$\underline{\text { Nuklearmedizinische Verfahren }}$}

Die Positronenemissionstomographie (PET) kann mithilfe der Indikatorsubstanz ${ }^{18}$ F-FluorDesoxyglucose (FDG) den zerebralen Glukosemetabolismus sichtbar machen. Im Falle der AD zeigt sich eine Minderaufnahme im Temporal- und Parietallappen sowie im hinteren Gyrus cinguli (Jagust 2006). Durch die Entwicklung neuer Indikatoren, z.B. des sogenannten 
Pittsburgh Compound-B (PIB), einer Substanz, die spezifisch an Amyloid-Plaques bindet, ist es möglich, diese in-vivo darzustellen (Berti et al. 2010).

Die Single-Photon-Emissions-Tomographie (SPECT) ermöglicht die Messung des zerebralen Blutflusses. Bei der AD kann dabei typischerweise eine bilaterale temporo-parietale Minderperfusion beobachtet werden (Scheltens 2009).

\subsubsection{Biomarker im Liquor}

\section{$\underline{\text { Tau-Protein und phosphoryliertes Tau-Protein }}$}

Das Tau-Protein befindet sich physiologischerweise in neuronalen Axonen (Buee et al. 2000). Die für die charakteristische Pathologie der AD typischen Tangles enthalten eine abnorm phosphorylierte Variante des Tau-Proteins (Iqbal et al. 2000). Die Gesamtkonzentration im Liquor scheint die Intensität der neuronalen Degeneration bei chronischen neurodegenerativen Erkrankungen widerzuspiegeln. Im Vergleich zu gesunden Kontrollen ist diese Konzentration bei Patienten mit AD ungefähr dreimal so hoch (Blennow und Hampel 2003). Darüber hinaus konnte gezeigt werden, dass erhöhte Liquorkonzentrationen von Tau und Phospho-Tau (siehe unten) auch mit der Schwere und Häufigkeit von Symptomen bei der AD korrelieren (Wallin et al. 2006).

Auch von phosphoryliertem Tau (p-Tau) lassen sich erhöhte Liquorkonzentrationen bei Patienten mit AD zeigen (Blennow 2004). Der p-Tau-Wert ist dabei wahrscheinlich kein Marker für die Intensität der Neurodegeneration, sondern für den Phosphorylierungs-Status des Tau-Proteins und somit möglicherweise für die Formation von neurofibrillären Bündeln im Hirnparenchym, welche aus phosphoryliertem Tau bestehen (Zetterberg und Blennow 2008).

\section{$\underline{\beta \text {-Amyloid }}$}

Das $\beta$-Amyloid ist der Hauptbestandteil der senilen Plaques (Masters et al. 1985). Sowohl das Gesamt- $\beta$-Amyloid als auch die beiden Varianten $A \beta_{1-40}$ und $A \beta_{1-42}$, welche sich durch die Anzahl ihrer Aminosäuren unterscheiden, lassen sich im Liquor nachweisen. Bei der AD ist dabei eine Verminderung der Konzentration von $A \beta_{1-42}$ im Vergleich zu Kontrollen zu beobachten (Blennow 2004). Die Hypothese, dass die erniedrigten $A \beta_{1-42}$-Werte durch eine vermehrte Sequestration der Peptide in Plaques verursacht wird, führte bislang jedoch zu widersprüchlichen Ergebnissen, da auch bei Erkrankungen ohne A $\beta$-Plaques eine geringe Konzentrationsverminderung beobachtet werden konnte (Zetterberg und Blennow 2008). Für das Gesamt- $\beta$-Amyloid und das $A \beta_{1-40}$ ließen sich keine verminderten Konzentrationen im 
Liquor von AD-Patienten nachweisen (Blennow 2004). Die Verminderung des Quotienten $\mathrm{A} \beta_{1-42} / \mathrm{A} \beta_{1-40}(A \beta-$ Ratio $)$ scheint jedoch eine hohe diagnostische Bedeutung $\mathrm{zu}$ haben (Zetterberg und Blennow 2008).

\subsubsection{Pathogenese}

Die Symptomatik der Alzheimer-Demenz ist auf eine Degeneration von Nervenzellen und Synapsen zurückzuführen, die im Hippokampus und entorhinalen Kortex beginnt und im Verlauf auch zunehmend andere Kortexareale betrifft (Braak et al. 1999). Auf mikroskopischer Ebene lassen sich zwei charakteristische Merkmale nachweisen, die Alois Alzheimer bereits 1907 beschrieben hat (Alzheimer 1907). Es handelt sich hierbei um senile Plaques (SP) und um neurofibrilläre Bündel (Tangles, NFT). SP sind extrazelluläre Ablagerungen, deren Kern hauptsächlich aus unlöslichem $\beta$-Amyloid besteht (Masters et al. 1985). NFT liegen intrazellulär und enthalten hyperphosphoryliertes Tau-Protein (Iqbal et al. 2000). Aus pathobiochemischer Sicht kommt es bei der AD zu einem Defizit an Neurotransmittern. Im Vordergrund steht hier eine Störung des cholinergen Systems (Bartus et al. 1982). Die neurodegenerativen Prozesse beginnen wahrscheinlich bereits 20-30 Jahre vor den ersten klinischen Symptomen (Davies et al. 1988).

\subsubsection{1. $\beta$-Amlyloid}

Die heutzutage bekannteste Hypothese für die Pathogenese der AD ist die „AmyloidKaskaden-Hypothese" (siehe Abb. 2), die vor allem durch die Erforschung der genetischen Mutationen der AD Bestätigung fand (siehe Kapitel 1.3). Sie besagt, dass ein Ungleichgewicht zwischen der Produktion und dem Abbau von $\beta$-Amyloid (A $\beta)$, insbesondere $A \beta_{1-42}$, das initiale Ereignis einer Kaskade ist, die schließlich zur neuronalen Degeneration führt (Hardy und Selkoe 2002).

Beim $\beta$-Amyloid handelt es sich um ein - auch im physiologischen Stoffwechsel vorkommendes - Abbauprodukt des Amyloid-Precursor-Proteins (APP, Haass et al. 1992). Letzteres ist ein membranständiges Protein, welches durch drei Enzymgruppen prozessiert wird (Hardy und Selkoe 2002; Blennow et al. 2006; Querfurth und LaFerla 2010): Die $\alpha$ Sekretase bewirkt eine proteolytische Spaltung innerhalb der A $\beta$-Region des APP und wirkt somit einer Bildung von $\mathrm{A} \beta$ entgegen (nicht-amyloidogener Weg). Die $\beta$-Sekretase dagegen ruft eine Teilung des APP vor der A $\beta$-Region hervor, das entstehende Restprotein wird anschließend durch die $\gamma$-Sekretase in das plaquebildende $4 \mathrm{kDa}$ schwere $\beta$-Amyloid 
gespalten (amyloidogener Weg). Der Abbau von A $\beta$ geschieht unter normalen Bedingungen durch diverse Peptidasen (insulin-degrading-enzyme, neprilysin, endothelin-convertingenzyme) oder den LDL-Rezeptor. Das vermehrte Auftreten von $\mathrm{A} \beta$ bei der AD resultiert zum einen aus einer Verschiebung zum amyloidogenen Weg, zum anderen aus einer Störung des $\beta$-Amyloid-Abbaus.

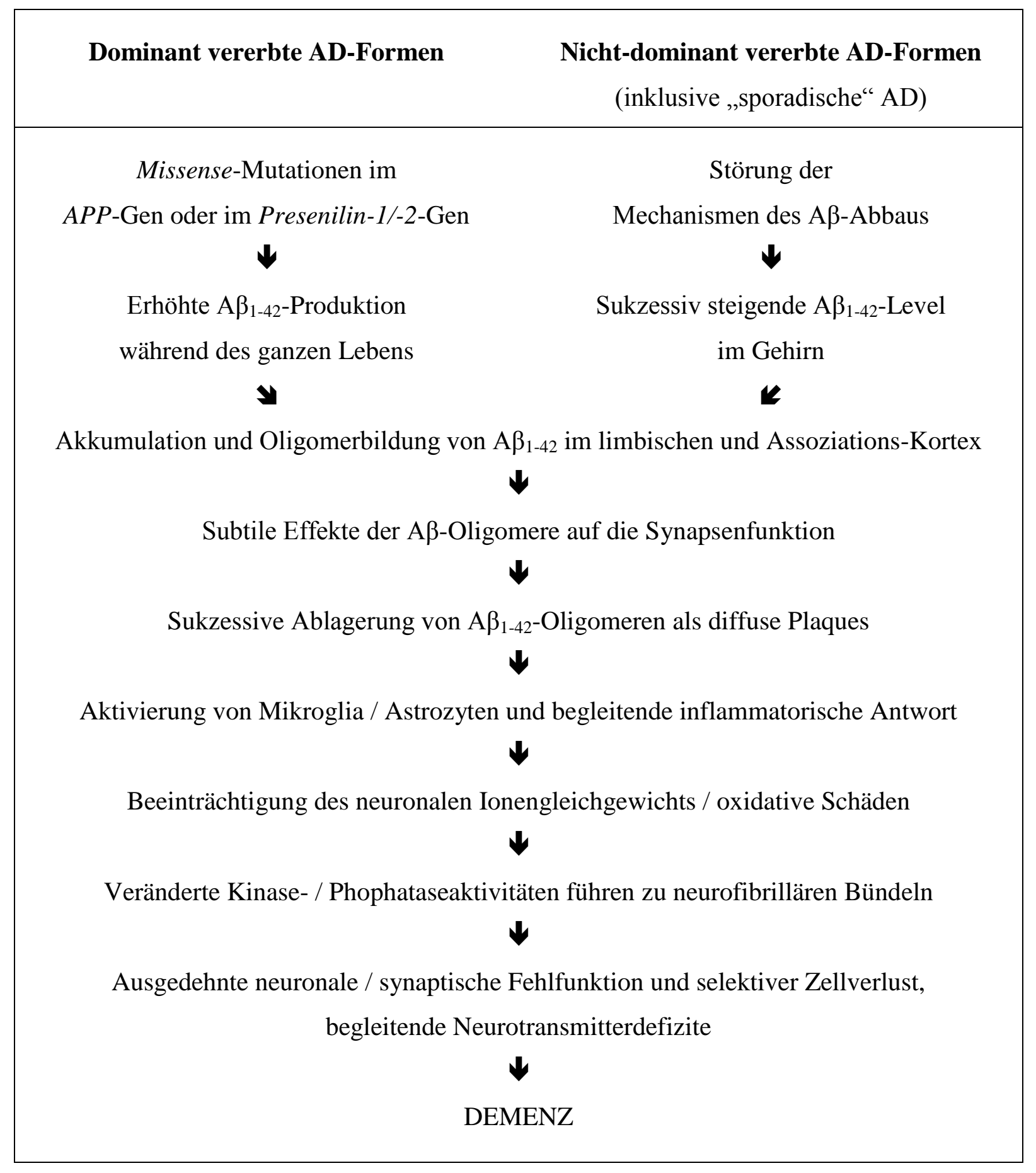

Abb. 2: Die Amyloid-Kaskadenhypothese (modifiziert nach: Selkoe 2008, S. 253) 
Die $\gamma$-Sekretase, deren katalytischer Kern aus Präsenilin besteht, bewirkt im letzten Schritt der $\mathrm{A} \beta$-Produktion sowohl die Entstehung von $\mathrm{A} \beta_{1-40}$ als auch $\mathrm{A} \beta_{1-42}$, die sich in der Anzahl der

Aminosäuren unterscheiden. Während im physiologischen Zellstoffwechsel $A \beta_{1-40}$ vorherrscht, kehrt sich im Falle der AD das Verhältnis zugunsten $A \beta_{1-42}$ um. Die $A \beta$-Peptide können sich einerseits in SP zusammenlagern oder zu löslichen Oligomeren aggregieren. Letztere scheinen durch Störung der Synapsenfunktion die eigentlichen neurotoxischen Agenzien zu sein und gut mit der Schwere der Krankheit zu korrelieren. Die bei der AD vermehrt vorliegenden $\mathrm{A} \beta_{1-42}$-Peptide neigen eher dazu, zu aggregieren und lösliche Oligomere zu bilden (Blennow et al. 2006; Querfurth und LaFerla 2010).

Die A $\beta$-Ablagerungen führen im weiteren Verlauf zu einer inflammatorischen Antwort durch Aktivierung von Mikroglia und Astrozyten, einer Beeinträchtigung des Ionengleichgewichts sowie zu oxidativem Stress. Am Ende der Kaskade stehen eine Fehlfunktion von Neuronen und Synapsen, Nervenzellverlust und ein Defizit an Neurotransmittern (Selkoe 2008).

\subsubsection{Tau-Protein}

Tau-Proteine werden in neuronalen Axonen exprimiert und sind an der Stabilisierung der Mikrotubuli beteiligt. Eine pathologische, vermehrte Phosphorylierung des Tau-Proteins vermindert die Bindung zu den Miktotubuli und führt zu einer Aggregation in gepaarten, helikal gewundenen Filamenten (paired helical filaments, PHF), die sich ihrerseits zu größeren Bündeln (Tangles) zusammenlagern. Sowohl durch die Destabilisierung der Mikrotubuli als auch durch die Tangle-Bildung kommt es zu einem gestörten axonalen Transport und damit zu einer Fehlfunktion von Neuronen und Synapsen (Buee et al. 2000).

Die Anzahl der NFT korreliert besser mit dem Nervenzellverlust und der Schwere der ADSymptomatik als die Amyloid-Plaques (Gomez-Isla et al. 2008).

Die Wechselwirkungen zwischen den beiden pathogenetischen Mechanismen sind nicht geklärt. Es wird vermutet, dass A $\beta$-Moleküle das Enzym Glycogen-Synthase-Kinase 3 (GSK 3) aktivieren können, welches wiederum imstande ist, die Tau-Proteine zu phosphorylieren (Querfurth und LaFerla 2010).

\subsubsection{Veränderungen der Neurotransmitter}

Die AD zeichnet sich durch eine Störung zahlreicher Neurotransmittersysteme aus. Die im Vordergrund stehende Verminderung von Acetylcholin lässt sich gemäß der ,,cholinergen Hypothese“ (Bartus et al. 1982; Bartus 2000; Terry und Buccafusco 2003) auf den drastischen 
Nervenzellverlust der basalen Vorderhirnstrukturen, insbesondere des Nucleus basalis Meynert, zurückführen. Letzterer besteht zu 90\% aus cholinergen Neuronen und weist neben Projektionen in nahezu alle Bereiche des Neokortex auch Afferenzen zum limbischen System und somit zum Hippokampus auf. Das hieraus resultierende cholinerge Defizit trägt somit laut Hypothese substantiell zu den Aufmerksamkeits- und Gedächtnisstörungen bei. In der symptomatischen Therapie der AD steht daher der Einsatz von Cholinesterasehemmern derzeit im Mittelpunkt (siehe auch Kapitel 1.2.7).

\subsubsection{Therapie}

Die Therapie der AD ist bislang nur symptomatisch möglich. Sogenannte „,disease-modifying drugs “, also den Krankheitsverlauf beeinflussende Therapeutika, sind in der Entwicklung und konnten teilweise in Tiermodellen Erfolge aufweisen.

\subsubsection{Cholinesterasehemmer}

Der Einsatz von Cholinesterasehemmern beruht auf der „,cholinergen Hypothese“ der AD (Bartus et al. 1982; Bartus 2000; Terry und Buccafusco 2003). Durch Hemmung der für den Abbau von Acetylcholin zuständigen Enzyme Acetylcholinesterase und Butylcholinesterase wird daher versucht, das bei der AD vorliegende cholinerge Defizit auszugleichen. Derzeit stehen in Deutschland mit Donepezil, Rivastigmin und Galantamin drei Wirkstoffe dieser Klasse zur Verfügung, die alle zur Behandlung der leichten bis mittleren AD zugelassen sind.

\subsubsection{Memantine}

Der NMDA-Rezeptor-Antagonist Memantine ist in Deutschland zur Behandlung der mittleren bis schweren AD zugelassen. Der Einsatz des Wirkstoffs geht auf die Annahme zurück, dass bei der $\mathrm{AD}$ erhöhte Konzentrationen des exzitatorischen Neurotransmitters Glutamat eine ständige Aktivierung des NMDA-Rezeptors und damit eine neuronale Zellschädigung auslösen (Greenamyre und Young 1989). Memantine soll als nicht-kompetitiver Antagonist diesen neurotoxischen Effekt verhindern, ohne die für kognitive Funktionen wichtige physiologische Aktivierung des NMDA-Rezeptors zu blockieren (Wilcock 2003). 


\subsubsection{Präventive Maßnahmen}

Trotz vielversprechender Ansätze aus epidemiologischen Studien reichen die Erkenntnisse zum gegenwärtigen Zeitpunkt jedoch noch nicht aus, um allgemeingültige Aussagen über die Effektivität präventiver medikamentöser Maßnahmen (z.B. Antihypertensiva, Statine, Antiinfectiva) oder den genauen Wirkungsgrad einer ausgeglichenen Ernährung und eines aktiven, gesunden Lebensstils zu treffen (Qiu et al. 2009)

\subsection{Genetik der Alzheimer-Demenz}

Bei der $\mathrm{AD}$ handelt es sich um eine genetisch heterogene Erkrankung, die anhand eines dichotomen Modells in eine meist früh auftretende, sehr seltene familiäre Form und eine eher im späteren Alter auftretende, weitaus häufigere sporadische Variante eingeteilt werden kann (Tanzi 1999). Eine familiäre Häufung kann bei etwa $60 \%$ aller EOAD-Fälle beobachtet werden, bei $13 \%$ lässt sich ein autosomal-dominanter Erbgang nachweisen (Bird 2008). Ursächliche autosomal-dominante Mutationen konnten bislang in den drei Genen APP, PSEN1 und PSEN2 gefunden werden. Bei der nicht-familiären Late-Onset-Variante scheinen dahingegen genetische Polymorphismen, allen voran das ApoE-Gen, die Krankheitsentstehung im Sinne eines Risikofaktors lediglich $\mathrm{zu}$ beeinflussen, ein Mendel'sches Vererbungsmuster liegt hier nicht vor (Corder et al. 1993).

\subsubsection{Amyloid-Precursor-Protein (APP)}

Das APP-Gen befindet sich auf dem langen Arm des Chromsoms 21 und kodiert für ein gleichnamiges Typ-1-Membranprotein. Mutationen in diesem Gen begünstigen durch eine alternative Prozessierung von APP die Entstehung von $\beta$-Amyloid (Haass et al. 1994). Im Jahre 1991 wurde die erste APP-Missense-Mutation beschrieben (Goate et al. 1991), seitdem konnten laut der „Alzheimer Disease and Frontotemporal Dementia Mutation Database $(A D \& F T D M D B)$ “ über 30 weitere $\mathrm{AD}$-verursachende Mutationen im APP-Gen ausfindig gemacht werden (Cruts et al. 2012). Die meisten gefundenen Mutationen lagen dabei entweder in der A $\beta$-kodierenden Sequenz oder im Bereich der vermuteten Angriffsstellen der $\beta$ - und $\gamma$-Sekretasen, die für die amyloidogene Prozessierung des APP verantwortlich sind (Bertram und Tanzi 2008). APP-Mutationen können in ca. 15\% der Familien mit autosomaldominant vererbter AD gefunden werden (Campion et al. 1999). Das Erkrankungsalter liegt ungefähr bei 45 bis 61 Jahren (Chai 2007). 
Die Tatsache, dass sich bei Patienten mit Trisomie 21 bereits im frühen Alter die AD-typische Neuropathologie mit massiver Ablagerung von A $\beta$ im Hirnparenchym beobachten lässt, ist ebenfalls auf das APP-Gen zurückzuführen. Durch das Vorliegen einer zusätzlichen GenKopie kommt es hier zur erhöhten Expression und damit zu einer vermehrten Bildung von Amyloidplaques. Letztere kann bereits in der zweiten Dekade beginnen und mit dem Alter weiter ansteigen (Lemere et al. 1996).

\subsubsection{Präsenilin (PSEN) 1 und 2}

Beide PSEN-Gene kodieren für polytopische Membranproteine, die ähnlich wie APP in allen Zellen exprimiert werden (Selkoe 2008). Im Jahre 1995 gelang es erstmals, das auf dem langen Arm von Chromosom 14 lokalisierte PSEN-1 zu klonen und die ersten Mutationen zu beschreiben (Sherrington et al. 1995). Bis zum jetzigen Zeitpunkt wurden laut der „AD\&FTDMDB“ über 180 pathogenetische PSEN-1-Mutationen gefunden (Cruts et al. 2012). PSEN-1 scheint eine essentielle Rolle im $\gamma$-Sekretase-Komplex zu spielen, der an der Prozessierung des APP zu A $\beta$ beteiligt ist. Ob es sich beim PSEN-1 um einen Mediator für den Membrantransport (membrane trafficking) von APP handelt oder ob es direkt in die Prozessierung von APP durch die $\gamma$-Sekretase involviert ist, kann noch nicht sicher gesagt werden (Selkoe 2008). Patienten mit PSEN-1-Mutationen zeigen einen sehr frühen Krankheitsbeginn zwischen 30 und 55 Jahren (Cruts und Van Broeckhoven 1998).

Für das PSEN-2-Gen (Levy-Lahad et al. 1995), welches sich auf dem langen Arm von Chromosom 1 befindet, konnten bislang 13 pathogenetische Mutationen nachgewiesen werden (Cruts et al. 2012). Auch diesem Gen wird eine enzymatische Rolle im $\gamma$-SekretaseKomplex zugeschrieben (Bertram und Tanzi 2008). Der Erkrankungsbeginn bei PSEN-2Mutationen variiert stark (Sherrington et al. 1996), im Durchschnitt wird jedoch ein eher späterer Beginn und eine langsamere Progression als bei APP- und PSEN-1-Mutationen beobachtet (Bertram 2008).

Während PSEN2-Mutationen sehr selten sind, können Mutationen im PSEN1-Gen in ca. 56\% der Familien mit autosomal-dominant vererbter AD identifiziert werden (Campion et al. 1999).

\subsubsection{Apolipoprotein E (ApoE)}

ApoE ist ein 299 Aminosäuren langes Glycoprotein, welches hauptsächlich in der Leber und im Gehirn synthetisiert wird. In letzterem sind Astrozyten, Mikroglia und in geringerem Maße 
auch Neurone die Hauptproduzenten. Eine wichtige physiologische Aufgabe dieses Proteins ist die Funktion als Ligand in der rezeptorvermittelten Endozytose von Lipoproteinpartikeln (Kim et al. 2009).

Das gleichnamige kodierende Gen ist auf Chromosom 19 lokalisiert und existiert in drei Isoformen ( $\varepsilon 2, \varepsilon 3$ und $\varepsilon 4$ ) (Bekris et al. 2010). Während das $\varepsilon 2$-Allel mit einem erniedrigten Risiko für eine $\mathrm{AD}$ assoziiert ist, stellt das $\varepsilon 4$-Allel einen starken genetischen Risikofaktor für den Late-Onset-Typ dar. Es konnte hierbei gezeigt werden, dass sich im Vergleich zu Individuen ohne $\varepsilon 4$-Allel das Risiko für Heterozygote um den Faktor drei und für Homozygote sogar um den Faktor fünfzehn erhöht (Corder et al. 1993). Im Gegensatz zu den familiären Mutationen führt die Anwesenheit von $\varepsilon 4$ also nicht zwingend zur Erkrankung, sondern modifiziert dosisabhängig lediglich das Risiko. Diese Beobachtungen lassen sich am ehesten auf eine Isoform-spezifische Bindungsaffinität von ApoE und $\mathrm{A} \beta$ zurückführen, die möglicherweise Zeitpunkt, Ort und Grad der A $\beta$-Akkumulation beeinflusst (Strittmatter et al. 1993). So wiesen zahlreiche Studien einen Zusammenhang zwischen ApoE \&4 und einer vermehrten $A \beta$-Ablagerung sowohl im Hirnparenchym als auch in zerebralen Gefäßen im Sinne einer Amyloid-Angiopathie nach (Rebeck et al. 1993; Schmechel et al. 1993; Greenberg et al. 1995; Hyman et al. 1995). Es wird vermutet, dass bei dieser Akkumulation eine durch ApoE vermittelte gestörte A $\beta$-Clearance eine zentrale Rolle spielt (Mawuenyega et al. 2010).

\subsubsection{Andere Suszeptibilitätsgene}

Infolge des technischen Fortschrittes konnte in den letzten Jahren insbesondere durch sogenannte „Genome-wide association studies“ (GWAS) eine Vielzahl möglicher neuer Suszeptibilitätsgene für die AD gefunden werden. Trotz der vielversprechenden Ergebnisse stellten sich die neuen Kandidatengene in den unterschiedlichen Studien jedoch weitgehend als recht inkonsistent dar. Die Assoziation des Genlocus von ApoE ließ sich dagegen in fast allen Studien reproduzieren und zeigte eine weitaus höhere Signifikanz als die neuen Loci (Bertram und Tanzi 2009). Aufgrund der sehr großen, stetig wachsenden Anzahl an genetischen Studien hat die Forschungsgruppe um Bertram et al. eine kontinuierlich aktualisierte Datenbank erstellt, die eine Metaanalyse aller zugänglichen Assoziationsstudien beinhaltet (Bertram et al. 2007, www.alzgene.org). Tabelle 2 zeigt die Gene mit der stärksten Assoziation (Stand Februar 2010). 
Tabelle 2: Risikogene und Odds Ratio, Stand Februar 2010 (Bertram et al. 2007)

\begin{tabular}{lll}
\hline Gen & Polymorphismus & Odds Ratio \\
\hline ApoE $\varepsilon 2 / 3 / 4$ & ApoE $\varepsilon 2 / 3 / 4$ & $3,68(\varepsilon 4$ vs. $\varepsilon 3)$ \\
$C L U$ & rs11136000 & 0,89 (A vs. G) \\
PICALM & rs541458 & 0,88 (C vs. T) \\
EXOC3L2 & rs597668 & 1,17 (C vs. T) \\
BIN1 & rs744373 & 1,17 (G vs. A) \\
GWA 14q32.13 & rs11622883 & 0,84 (A vs. T) \\
TNK1 & rs1554948 & $0,84($ A vs. T) \\
IL8 & rs4073 & 1,27 (A vs. T) \\
LDLR & rs5930 & 0,85 (A vs. G) \\
$C S T 3$ & rs1064039 & 1,13 (A vs. G) \\
ACE & rs1800764 & 0,88 (C vs. T) \\
\hline
\end{tabular}

\subsection{Die ,rapid-progressive Alzheimer-Demenz“6 (rpAD)}

\subsection{1. Überblick und Definition}

Die AD verläuft in den meisten Fällen langsam-progredient mit einer mittleren Überlebenszeit von 3-9 Jahren (Goldberg 2007; Querfurth und LaFerla 2010) und einem durchschnittlichen Verlust von 3 Punkten im MMST pro Jahr (Morris et al. 1993). In einigen Fällen werden jedoch auch rapid-progressive Verläufe mit deutlich verringerter Überlebenszeit und einem raschen MMST-Verfall beobachtet (Mann et al. 1989; Caselli et al. 1998; Van Everbroeck et al. 2004; Josephs et al. 2009; Schmidt et al. 2010). Bei diesen Patienten werden häufig ein verändertes klinisches Profil sowie abweichende Biomarker-Konstellationen beschrieben. Darüber hinaus werden unterschiedliche koexistierende Erkrankungen und diverse genetische Polymorphismen diskutiert, die möglicherweise einen Einfluss auf die Progredienz der AD haben. Da es bis zum jetzigen Zeitpunkt keine Einigkeit über den Begriff „,rapid“ gibt, fehlt bislang eine eindeutige Definition. Die Angaben in der Literatur bewegen sich zwischen $>3$ MMST-Punkten / Jahr (Carcaillon et al. 2007) und >8 MMST-Punkten / Jahr (Dumont et al. 2005). Tabelle 3 gibt einen Überblick über die verschiedenen Definitionen und die davon abhängige Häufigkeit von rpAD. 
Tabelle 3: Definitionen und Häufigkeiten von rpAD in Studien

\begin{tabular}{ccc}
\hline $\begin{array}{c}\text { Definition } \\
\text { Punktverlust im } \\
\text { MMST) }\end{array}$ & $\begin{array}{c}\text { Anteil rapider Fälle an } \\
\text { Studienpopulation }\end{array}$ & Autoren \\
\hline > 3 Punkte / Jahr & $33,9 \%$ & (Carcaillon et al. 2007) \\
$>$ 3 Punkte / Jahr & $30 \%$ & (Cortes et al. 2008) \\
$>$ 4,57 Punkte / Jahr & $11 \%$ & (Cortes et al. 2008) \\
$>$ 4,9 Punkte / Jahr & $8 \%$ & (Wallin et al. 2010) \\
$>$ 8 Punkte / Jahr & $24,8 \%$ & (Dumont et al. 2005) \\
\hline
\end{tabular}

\subsubsection{Klinik}

Ein schneller Krankheitsverlauf scheint mit bestimmten klinischen Symptomen assoziiert zu sein. Insbesondere extrapyramidal-motorische Zeichen (Scarmeas et al. 2004; Portet et al. 2009) sowie fokalneurologische Symptome (Tschampa et al. 2001; Van Everbroeck et al. 2004; Schmidt et al. 2010) sind Prädiktoren für eine rasche Progredienz der AD. In einigen beschriebenen Fällen konnte eine rpAD sogar das Erscheinungsbild der Creutzfeldt-JakobKrankheit imitieren (Tschampa et al. 2001; Reinwald et al. 2004; Van Everbroeck et al. 2004; Jayaratnam et al. 2008; Josephs et al. 2009; Schmidt et al. 2010). Es liegen des Weiteren Hinweise vor, dass auch eine starke psychotische Symptomatik (Wilkosz et al. 2010), eine Apathie (Starkstein et al. 2006) oder eine früh auftretende konstruktorische Apraxie (Smith et al. 2001) mit einem schnellen Krankheitsverlauf assoziiert sind.

\subsubsection{Komorbidität und Risikofaktoren}

Trotz eines wahrscheinlichen Effektes von kardiovaskulären Krankheiten und Diabetes mellitus auf die Entstehung der AD wird deren Einfluss auf die Progressionsgeschwindigkeit kontrovers diskutiert (Mielke et al. 2007; Abellan van Kan et al. 2009). Angaben über die Rolle des Ausbildungsgrades oder einer bereits schweren kognitiven Beeinträchtigung bei Erkrankungsbeginn liefern hinsichtlich der Auswirkung auf die Progression ebenfalls widersprüchliche Ergebnisse (Ito et al. 2010; Marra et al. 2000; Hui et al. 2003; Atchison et al. 2007). Als weitere potentielle Prädiktoren für eine $\mathrm{rpAD}$ werden ein früh einsetzender rapid-progressiver Verlauf (Soto et al. 2008a) sowie das männliche Geschlecht (Roselli et al. 2009) beschrieben. 


\subsubsection{Biomarker}

In mehreren Studien konnte gezeigt werden, dass ein schneller Verlauf der AD mit Veränderungen der Biomarker in der Zerebrospinal-Flüssigkeit einhergehen kann. So berichteten Snider et al., dass bei Individuen mit hohen Tau- oder Phospho-Tau-Werten und niedrigem $A \beta_{1-42}$-Level oder mit einer hohen Tau / $A \beta_{1-42}$-Ratio eine raschere Progression beobachtet werden konnte (Snider et al. 2009). Auch Wallin et al. kamen in ihrer Studie zu dem Schluss, dass AD-Patienten mit sehr hohem Tau und Phospho-Tau sowie niedrigem $\mathrm{A} \beta_{1-42}$ eine schnellere Progression der kognitiven Defizite, ein schlechteres Ansprechen auf Cholinesterasehemmer sowie eine höhere Mortalität aufzeigen (Wallin et al. 2010). Kester et al. wiesen zudem auf die Assoziation zwischen raschem Verlauf und der Konstellation von hohem Tau ohne proportional erhöhtes Phospho-Tau hin (Kester et al. 2009). Tau und Phospho-Tau scheinen darüber hinaus auch Marker für die Schwere und Anzahl von Symptomen bei der AD zu sein (Wallin et al. 2006).

\subsubsection{Genetik}

Der potenzielle Einfluss genetischer Polymorphismen auf die Progressionsgeschwindigkeit wurde in diversen Studien untersucht. Die Ergebnisse sind in einigen Fällen jedoch widersprüchlich. Besonders die Rolle des für die Krankheitsentstehung der AD wichtigen ApoE-Gens ist noch unklar. Tabelle 4 gibt einen Überblick über Beispiele möglicher genetischer Prädiktoren der Progression. 
Tabelle 4: Einfluss genetischer Polymorphismen auf den Verlauf der AD (Beispiele)

\begin{tabular}{|c|c|c|c|}
\hline Gen/Polymorphismus & langsamer Verlauf & $\begin{array}{l}\text { kein Einfluss auf } \\
\text { Verlauf }\end{array}$ & schneller Verlauf \\
\hline$A p o E \varepsilon 4$ & & (Kester et al. 2009) & $\begin{array}{c}\text { (Cosentino et al. } \\
\text { 2008) }\end{array}$ \\
\hline kein $A p o E \varepsilon 4$ & & & (Schmidt et al. 2010) \\
\hline$\frac{\text { kein } A p o E}{(E O A D)} \varepsilon 4$ & & & $\begin{array}{c}\text { (van der Vlies et al. } \\
2009 \text { ) }\end{array}$ \\
\hline $\begin{array}{l}\text { BuChE } \\
\text { (K-Allel) }\end{array}$ & $\begin{array}{c}\text { (O'Brien et al. 2003), } \\
\text { (Holmes et al. 2005) }\end{array}$ & & \\
\hline $\begin{array}{l}\text { G51S PNP } \\
\text { (AA-Genotyp) }\end{array}$ & & & (Tumini et al. 2007) \\
\hline $\begin{array}{l}H M G C R \\
\text { (A-Allel) }\end{array}$ & & & $\begin{array}{c}\text { (Porcellini et al. } \\
\text { 2007) }\end{array}$ \\
\hline $\begin{array}{l}\text { PSEN1 } \\
\text { rs3025780 } \\
\text { (TG-Genotyp) } \\
\text { rs7152131 } \\
\text { (CA-Genotyp) } \\
\text { rs3025787 } \\
\text { (CG-Genotyp) }\end{array}$ & (Belbin et al. 2009) & & $\begin{array}{l}\text { (Belbin et al. 2009) } \\
\text { (Belbin et al. 2009) }\end{array}$ \\
\hline $\begin{array}{l}A C T 7 \\
\text { (GC- u. CC-Genotyp) }\end{array}$ & & & (Belbin et al. 2008) \\
\hline $\begin{array}{l}\text { ACT } 17 \\
\text { (AA-Genotyp) }\end{array}$ & & & (Kamboh et al. 2006) \\
\hline $\begin{array}{l}\text { ACT: Promotor- } \\
\text { Polymorphismus } \\
\text { (TT-Genotyp) } \\
+ \text { ApoE } \varepsilon 4\end{array}$ & & & (Licastro et al. 2005) \\
\hline $\begin{array}{l}I L-1 \alpha-889 \\
(* 1 / * 1-G e n o t y p)\end{array}$ & & & (Murphy et al. 2001) \\
\hline $\begin{array}{l}I L-18-137 \\
\text { (CC-Genotyp) }\end{array}$ & & & (Bossu et al. 2007) \\
\hline $\begin{array}{l}F A S-1377 \\
\text { (AG- u. GG-Genotyp) }\end{array}$ & & & $\begin{array}{c}\text { (Chiappelli et al. } \\
\text { 2006) }\end{array}$ \\
\hline $\begin{array}{l}\text { RAGE G82S } \\
\text { (GS- u. SS-Genotyp) }\end{array}$ & & & (Li et al. 2010) \\
\hline
\end{tabular}




\subsection{Fragestellung}

Die $\mathrm{AD}$ ist eine Erkrankung, bei der es $\mathrm{zu}$ einem komplexen Zusammenspiel unterschiedlicher genetischer Faktoren kommen kann. Während für die familiäre EOAD autosomal-dominante Mutationen in drei Genen gefunden werden konnten, ist bei der überwiegend auftretenden LOAD von einem genetisch komplexen Muster auszugehen. Der bislang stärkste bekannte Risikofaktor für die LOAD ist das ApoE- $\varepsilon 4-A l l e l$, dessen Einfluss in zahlreichen Studien bestätigt werden konnte. In den letzten Jahren konnte zudem eine Vielzahl neuer Kandidatengene entdeckt werden, die in sogenannten Genome-wide association studies (GWAS) eine signifikante Assoziation zur AD zeigten (siehe Tabelle 2, S. 15). Neben krankheitsassoziierten Genen wurden in letzter Zeit auch vereinzelt genetische Polymorphismen beschrieben, die möglicherweise einen Einfluss auf die Krankheitsprogression haben (siehe Tabelle 4, S. 18). Systematische Informationen darüber, inwieweit auch die neuen, in den GWAS gefundenen Suszeptibilitätsgene die Progression der Erkrankung beeinflussen, existieren bislang allerdings nicht. Auch für die sehr rasch verlaufenden $\mathrm{AD}$-Formen (rapid-progressive $\mathrm{AD}$ ) fehlt bislang eine systematische Untersuchung eines potenziellen Einflusses neuer GWAS-Kandidatengene.

In der vorliegenden Arbeit soll daher untersucht werden, ob und in welchem Maße die in GWAS-Studien gefundenen Suszeptibilitätsgene einen Einfluss auf die Progression der AD haben. Das Ziel der Bemühungen soll dabei insbesondere sein, mögliche genetische Prädiktoren für die rapid-progressive Form der AD zu finden. Zudem soll geprüft werden, ob sich die genetischen Einflüsse bei Frauen und Männern sowie bei Patienten mit EOAD und LOAD unterscheiden. „Störfaktoren“ wie die Erkrankungsdauer, das Krankheitsstadium oder auch die Ausbildungsdauer der Patienten sollen dabei berücksichtigt werden.

Zusammenfassend sind die zentralen Fragestellungen dieser Doktorarbeit folgende:

- Haben die neuen, in GWAS-Studien gefundenen Gene möglicherweise einen Einfluss auf die Krankheitsprogression der AD?

- Haben diese Kandidatengene eine prädiktive Funktion für die rapid-progressive Form der AD?

- Unterscheidet sich der potenzielle Einfluss dieser Gene auf die Krankheitsprogression bei Frauen und Männern?

- Gibt es einen Unterschied zwischen Patienten mit EOAD und LOAD hinsichtlich dieses möglichen Geneinflusses? 


\section{Patienten und Methoden}

\subsection{Studiendesign und Patientenrekrutierung}

\subsubsection{Hintergrund}

Im Nationalen Referenzzentrum (NRZ) für die Surveillance Transmissibler Spongiformer Enzephalopathien der Universitätsmedizin Göttingen gehen täglich Liquorproben und Verdachtsmeldungen bezüglich Prionerkrankungen aus ganz Deutschland ein. Im Vordergrund steht dabei die epidemiologische Erfassung der Creutzfeldt-Jakob-Erkrankung (CJK), die sich durch eine schnell-verlaufende Demenz sowie eine Fülle an fokalneurologischen Symptomen auszeichnet. Die häufigste Differenzialdiagnose der Creutzfeldt-Jakob-Erkrankung ist die AD (Zerr und Poser 2002; Heinemann et al. 2007). Durch eine retrospektive Studie konnte gezeigt werden, dass die als CJK-Verdachtsfall gemeldeten Fälle, die sich differenzialdiagnostisch als AD herausstellten, einen besonders rapiden Verlauf aufwiesen (Schmidt et al. 2010). Mit dem Ziel der weitergehenden Untersuchung und Charakterisierung dieser rasch verlaufenden AD-Fälle wurde eine klinische Studie konzipiert. Diese sogenannte rpAD-Studie (rapid-progressive Alzheimer Demenz) stellt die Grundlage für die vorliegende Arbeit dar.

\subsubsection{Die rpAD-Studie}

Die rpAD-Studie ist eine unizentrische longitudinale Studie und wird im NRZ seit Juli 2008 durchgeführt. Abb. 3 gibt einen schematischen Überblick über die Studie. Die Durchführung wird in Kapitel 2.3 beschrieben. 


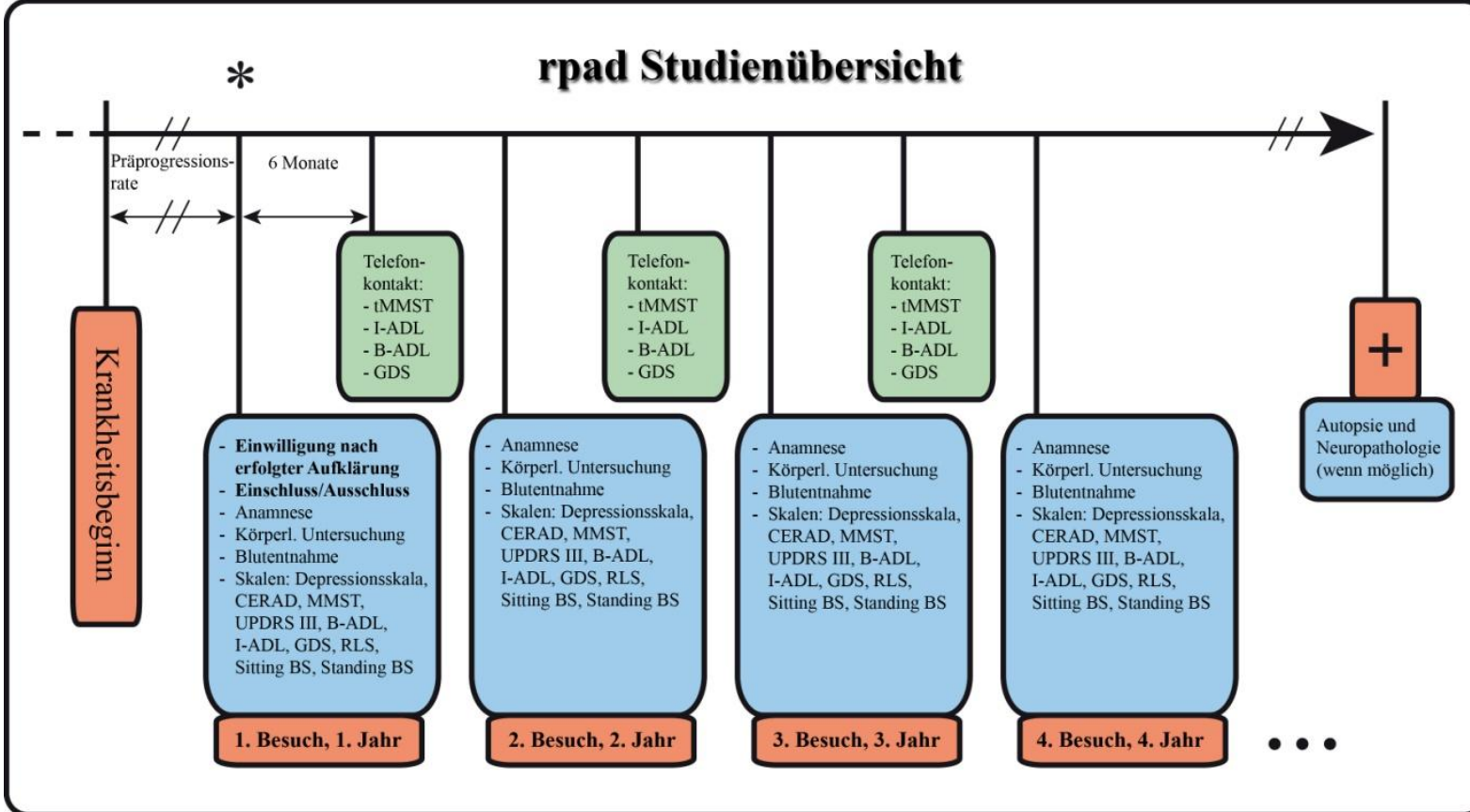

Abb. 3: Aufbau der rpAD-Studie

\subsubsection{Patientenrekrutierung}

Die Rekrutierung von Patienten für die rpAD-Studie fand überwiegend im Rahmen der epidemiologischen Erfassung von Prionerkrankungen im NRZ der Universitätsmedizin Göttingen statt. Krankenhäuser, die den Verdacht auf eine Prionerkrankung äußerten, wurden gebeten, Liquorproben, klinische Angaben sowie neuroradiologische und EEG-Befunde des jeweiligen Patienten an das NRZ zu senden. In Zusammenschau der Befunde wurde der Patient schließlich von den in der Prionepidemiologie tätigen Ärzten klassifiziert. Stellte sich dabei die Diagnose einer Prionerkrankung als unwahrscheinlich heraus und deuteten die Angaben eher auf eine AD hin, wurde der behandelnde Arzt des jeweiligen Krankenhauses über die rpAD-Studie informiert. Zudem wurde dieser gebeten, sowohl den Patienten als auch die Angehörigen über die Möglichkeit der Studienteilnahme in Kenntnis zu setzen. Im Falle des Teilnahmewunsches wurden diese schließlich vor Ort von einem Studienarzt besucht.

Darüber hinaus wurden auch Patienten aus der Neurologischen und Psychiatrischen Klinik der Universitätsmedizin Göttingen rekrutiert, bei denen im Rahmen eines stationären Aufenthaltes der Verdacht auf eine AD gestellt wurde. Diese wurden von den behandelnden Ärzten über die Möglichkeit der Studienteilnahme informiert. Bestand ein Interesse zur 
Partizipation, wurden dem Patienten bzw. dessen Angehörigen durch einen Arzt der rpADStudie Umfang und Ablauf der Studie eingehend vor Ort erläutert.

\subsubsection{Aufklärung und Einwilligung}

Die Studie wurde von der an der Universitätsmedizin Göttingen ansässigen Ethikkommission genehmigt (Antragsnummer 9/6/08). Die Bestimmungen des Datenschutzes (Good Clinical Practice, Gendiagnostikgesetz) wurden beachtet. Zur Teilnahme an der Studie wurden sowohl der Patient als auch dessen Angehörigen bzw. Betreuer sorgfältig aufgeklärt. Des Weiteren war eine schriftliche Einwilligung des Patienten Voraussetzung für eine Teilnahme. Im Falle einer eingeschränkten Einwilligungsfähigkeit des Patienten wurden Betreuer oder nahe Angehörige herangezogen. Separate Aufklärungs- und Einwilligungsbögen standen hierfür zur Verfügung (siehe Anhang, S. 82-85).

\subsubsection{Einschlusskriterien}

Folgende Punkte mussten für einen Einschluss in die Studie erfüllt sein:

- Revidierte McKhann-Kriterien (McKhann et al. 1984) zur Diagnose einer AD, modifiziert nach Dubois (Dubois et al. 2007, siehe Anhang, Tabelle 23, S. 81)

- Bereitschaft des Patienten, der Angehörigen / der Betreuer und des Hausarztes zur Teilnahme

- Schriftliche Einwilligung des Patienten oder der Angehörigen / der Betreuer bezüglich der Teilnahme an der Studie inklusive Blutentnahmen und Messung von zuvor festgelegten Parametern in Blut und Liquor

- Erlaubnis des Patienten bzw. der Angehörigen / der Betreuer für den Hausarzt, Informationen an die Studienärzte übermitteln zu dürfen.

\subsubsection{Ausschlusskriterien}

Als Ausschlusskriterien dienten die in den revidierten Kriterien nach Dubois festgehaltenen Punkte (Dubois et al. 2007, siehe Anhang, Tabelle 23, S. 81). 


\subsection{Erhobene und gemessene Parameter}

\subsubsection{Anamnese und Untersuchung}

Die Erhebung der Anamnese sowie der körperlichen internistischen und neurologischen Untersuchung erfolgte mit Hilfe des in der Neurologischen Klinik der Universitätsmedizin Göttingen verwendeten Aufnahmebogens. Zusätzlich kamen standardisierte Fragebögen zum Einsatz (siehe Anhang, S. 86-91), die folgende Punkte beinhalteten:

- Genauer Beginn der demenziellen Symptomatik des Patienten (semistrukturelle Angehörigenbefragung)

- Größe / Gewicht des Patienten

- Bildungsgrad des Patienten

- Symptome seit Krankheitsbeginn

- Vorerkrankungen

- Aktuelle Medikation

- Familienanamnese

- Stammbaum bei auffälliger Familienanamnese.

\subsubsection{Neuropsychologische Tests}

Zur neuropsychologischen Diagnostik der AD wurde in der vorliegenden Arbeit die CERADPlus-Testbatterie verwendet. Der in dieser enthaltene Mini-Mental-Status-Test diente als Verlaufsparameter.

\subsubsection{Mini-Mental-Status-Test (MMST)}

Der MMST stellt das wahrscheinlich bekannteste Verfahren zur orientierenden Schweregradeinschätzung kognitiver Störungen dar (Folstein et al. 1975). Der Test beinhaltet unterschiedliche Fragen und Aufgaben, die sich ausschließlich auf die kognitiven Aspekte des mentalen Status konzentrieren. Kognitive Domänen, die mithilfe dieses Instrumentes geprüft werden, sind u.a. zeitliche und örtliche Orientierung, Merkfähigkeit, Aufmerksamkeit, Sprache sowie konstruktive Praxis. Die Durchführung des Tests erfordert etwa 5-10 Minuten. Die Gesamtpunktzahl, die im MMST erreicht werden kann, beträgt 30 Punkte. 


\subsubsection{CERAD-Testbatterie}

Die vom amerikanischen Consortium for the Establishment of a Registry for Alzheimer's Disease (CERAD) entwickelte standardisierte Testbatterie (Morris et al. 1988; Morris et al. 1989) setzt sich aus insgesamt 8 Untertests zusammen. Diese erfassen gezielt Hirnleistungsstörungen, die bei der $\mathrm{AD}$ im Vordergrund stehen. Die Durchführung der 8 Untertests dauert etwa 30-45 Minuten. Eine deutschsprachige Version der CERADTestbatterie wurde von der Geriatrischen Universitätsklinik Basel entwickelt, an einer Stichprobe von 614 gesunden Patienten normiert und im Rahmen einer Multicenterstudie validiert (Thalmann et al. 2000; Aebi 2002). Im Zuge der deutschsprachigen Normierung wurden Formeln für die Berücksichtigung von Alter, Geschlecht und Ausbildung entwickelt. Die Ergebnisse („Rohwerte“) jedes Untertests werden dabei in demographisch angepasste ,zWerte" transformiert. Diese zeigen schließlich für jede Aufgabe an, wie weit die Testperson vom Durchschnitt der Normalpopulation abweicht (Aebi 2002). Als untere Grenze des Normalbereichs wurde ein z-Wert von -1,65 festgelegt, welcher dem 5. Perzentil entspricht. In Tabelle 5 ist eine Übersicht der verschiedenen Aufgaben und deren Beschreibung dargestellt.

Tabelle 5: Untertests der CERAD-Testbatterie

\begin{tabular}{ll}
\hline Name des Tests & Beschreibung \\
\hline 1. Semantische Wortflüssigkeit & Aufzählung von möglichst vielen Begriffen \\
& $\begin{array}{l}\text { aus der Kategorie 'Tiere' (Zeitbegrenzung: } 1 \\
\text { Minute) }\end{array}$ \\
2. Boston Naming Test (modifiziert) & Benennung von 15 Strichzeichnungen in 3 \\
& Schwierigkeitsstufen \\
3. Mini-Mental-Status-Test & Siehe Kapitel 2.2.2.1 \\
4. Wortliste Gedächtnis & Merken und anschließendes Abrufen von 10 \\
& Begriffen (3 Durchgänge) \\
5. Konstruktive Praxis & Abzeichnen von 4 Figuren \\
6. Wortliste Abrufen & Erneutes Abrufen der Wortliste \\
7. Wiedererkennung & Unterscheidung der Begriffe der Wortliste \\
8. Konstruktive Praxis Abrufen & von 10 neuen Begriffen \\
& Erneutes Zeichnen der Figuren aus Aufgabe \\
\hline
\end{tabular}


Tabelle 5 (Fortsetzung)

\begin{tabular}{cl}
\hline Name des Tests & Beschreibung \\
\hline 9. Trailmaking-Test A* & Verbinden von Zahlen in aufsteigender \\
& Reihenfolge \\
10. Trailmaking-Test B* & Verbinden von Zahlen und Buchstaben in \\
& aufsteigender Reihenfolge \\
11. Phonematische Wortflüssigkeit* & Aufzählung von möglichst vielen Begriffen \\
& mit dem Anfangsbuchstaben 's‘ \\
& (Zeitbegrenzung: 1 Minute) \\
\hline
\end{tabular}

* nur im CERAD-plus-Test enthalten

\subsubsection{Liquordiagnostik}

Die Bestimmung der Liquormarker erfolgte durch Mitarbeiter des Liquorlabors des NRZ der Universitätsmedizin Göttingen. Mit Ausnahme des 14-3-3-Proteins wurden alle Messungen mithilfe von ELISA-Tests durchgeführt (Enzyme-linked immunosorbent assay).

\subsubsection{Gesamt-Tau}

Für die Messung des bei der AD erhöhten Tau-Proteins wurde in der vorliegenden Arbeit der Innotest ${ }^{\circledR}$ hTau-Ag-ELISA der Firma Innogenetics (Gent, Belgien) benutzt. Der Standardbereich der messbaren Tau-Konzentration liegt zwischen 75 und 1200 pg/ml. Werte über $450 \mathrm{pg} / \mathrm{ml}$ wurden als erhöht festgelegt.

\subsubsection{Phospho-Tau}

Die Messung der bei der AD erhöhten Phospho-Tau-Konzentration wurde mit dem Innotest ${ }^{\circledR}$ Phospho-Tau ${ }_{(181 P)}$-ELISA der Firma Innogenetics (Gent, Belgien) durchgeführt. Der Standard-Messbereich liegt zwischen 15,6 und 500 pg/ml. Als Grenzwert wurde eine Konzentration von $61 \mathrm{pg} / \mathrm{ml}$ ermittelt (cut off).

\subsubsection{3. $\beta$-Amyloid}

Für die $\mathrm{A} \beta_{1-42}$-Messung kam der Innotest ${ }^{\circledR} \beta$-Amyloid -1-42 -ELISA der Firma Innogenetics (Gent, Belgien) zum Einsatz. Der Standardbereich der messbaren $A \beta_{1-42}$-Konzentration liegt zwischen 125 und $2000 \mathrm{pg} / \mathrm{ml}$. Werte unter $450 \mathrm{pg} / \mathrm{ml}$ wurden als erniedrigt definiert und dienten damit als Hinweis für eine AD. 
Für die Bestimmung der $\mathrm{A} \beta_{1-40}$-Konzentration wurde der hAmyloid- $\beta 40$-ELISA der Firma The Genetics Company, Inc. (Schlieren, Schweiz) verwendet. Der Standard-Messbereich liegt für diesen Test zwischen 100 und 2000 pg/ml. Die $A \beta_{1-40}$-Konzentration diente zur Berechnung $\operatorname{der} A \beta$-Ratio.

Diese berechnet sich wie folgt:

$$
A \beta \text {-Ratio }=A \beta_{1-42} / A \beta_{1-40} \times 10
$$

Eine A $\beta$-Ratio unter 1,0 wurde dabei als erniedrigt festgelegt und stellte somit einen Hinweis auf eine AD dar.

\subsubsection{Protein 14-3-3}

Zum Nachweis des Proteins 14-3-3 wurde ein Westernblot mit polyklonalen IgG-Antikörpern (14-3-3 $\beta$, Kaninchen) der Firma Santa Cruz Biotechnology (Santa Cruz, Kalifornien, USA) angewandt.

\subsubsection{Genetische Marker}

Die Bestimmung der genetischen Polymorphismen erfolgte in der Abteilung für klinische Chemie der Universitätsmedizin Göttingen. Die Auswahl der genetischen Marker, die für die vorliegende Arbeit analysiert wurden, richtete sich nach einer von der Alzgene-Datenbank veröffentlichten Liste der Kandidatengene mit der höchsten Assoziation zur AD (Stand Februar 2010, www.alzgene.org, Bertram et al. 2007). Berücksichtigt wurden nur Gene aus Studien, die kaukasische Populationen beinhalteten. Die ersten 11 Polymorphismen der Rangliste, die diese Kriterien erfüllten, wurden in der vorliegenden Arbeit untersucht. Alle PCR-Analysen wurden mithilfe eines LightCycler ${ }^{\circledR} 480$ (Real-Time-PCR-System) in 96-wellPCR-Platten der Firma Roche Molecular Diagnostics (Pleasanton, Kalifornien, USA) durchgeführt. Für das Design der Oligonukleotid-Proben wurde eine spezielle Software verwendet (Schütz und von Ahsen 1999). Der folgenden Tabelle 6 können Angaben zu den 11 untersuchten Polymorphismen und den verwendeten Primern entnommen werden. Bei allen Polymorphismen wurde das Vorliegen des Hardy-Weinberg-Gleichgewichts (HWG) als Qualitätsmarker für die Genotypisierung überprüft. Bei einem $p$-Wert > 0,05 wurde davon ausgegangen, dass die Polymorphismen im HWG lagen. Wie in Tabelle 7 ersichtlich, wurde das HWG bei keinem der überprüften Marker verletzt. 
Tabelle 6: Primer der untersuchten Polymorphismen (Angaben jeweils als 5`-Sequenz-3 ${ }^{`}$ )

\begin{tabular}{|c|c|c|c|c|c|}
\hline Gen & $\begin{array}{l}\text { Poly- } \\
\text { morphismus }\end{array}$ & Forward Primer & Reverse Primer & Anchor (5'Cy5 3'P) & Probe (3‘'Flu) \\
\hline BIN1 & rs744373 & AAGGCTGAAGGGAAAGTTGC & GCTGAGGGATCTCACCTTTG & CTGTCCCTGGTGCCTAGGT & AGAGCCTCAGACCTG \\
\hline IL8 & rs 4073 & CTTGTTCTAACACCTGCCACTC & CAAACCTGAGTCATCACACTTC & $\begin{array}{l}\text { AATTGTGGAGCTTCAGTATTTTAAAT } \\
\text { GTATAT }\end{array}$ & $\begin{array}{l}\text { AGCATACATTTGATAATT } \\
\text { CACC }\end{array}$ \\
\hline$C L U$ & rs11136000 & GGCTGCAGACTCCCTGAATC & TCCCAAAGTGCTGGGATTAC & $\begin{array}{l}\text { TTGCCACTTGACCCAATAATTCTGTA } \\
\text { AGA }\end{array}$ & AAACTCTCTAACGGGCC \\
\hline PICALM & rs541458 & CAAAATTGAAGCCCTTGTTG & GATTTGAGAGGAGGCTGGTG & $\begin{array}{l}\text { CAGGGATTGGTTTAAATTTGTCAACT } \\
\text { AG }\end{array}$ & TGCACA-ATTAGTAGCCAGT \\
\hline GWA14g32.13 & rs11622883 & TTTGCAGACGAGAAAACAGC & TTTGCTGAAGCCAACAACTG & AATGCGAGGAAGCAAAGAGCC & TCGGCGTGGAAGTTT \\
\hline$A C E$ & rs1800764 & TCAATTTCAGGCATCCATTG & AGGCAGAGATTGTGGTGAGC & $\begin{array}{l}\text { GTACATACTTTGCAAATATCTTCTCT } \\
\text { CATCCCA }\end{array}$ & ATAAGGGGGGGCETGC \\
\hline TNK1 & rs1554948 & CCTACCCTGAGCTCACCATC & TCCAGGTCCTCAGGCTTTAC & CCAGTAAAACTGGGCCAACT & AGCTCCTCAAAGGATGG \\
\hline$L D L R$ & rs5930 & СТTCTCTCСТCСТGCСТCAG & CTCTTCACGCCCTTGGTATC & GTCATAGGAAGAGACGCCGTGG & TCECCTGCTGATGACGG \\
\hline ApoE & $\varepsilon 2 / 3 / 4$ & AAGGCCTACAAATCGGAACT & CACGCGGCCCTGTTCCAC & $\begin{array}{l}\text { Codon 112: } \\
\text { GCCGCCTGGTGCAGTACCGC }\end{array}$ & $\begin{array}{l}\text { Codon 112: } \\
\text { CATGGAGGACGTGCGC }\end{array}$ \\
\hline & & & & $\begin{array}{l}\text { Codon 158: } \\
\text { GACCTGCAGAAGCGCCTGGC }\end{array}$ & $\begin{array}{l}\text { Codon 158: } \\
\text { GCTGCGTAAGCGGCTCCT } \\
\text { CCGCGATGCCG }\end{array}$ \\
\hline EXOC $3 L 2$ & rs597668 & AATGGACCACATCACTAAATGC & TCTCCCACTGTTTGGCTTATC & $\begin{array}{l}\text { ATAAATTAGATTCCATCAAAATTAA } \\
\text { AAACTTCTG }\end{array}$ & $\begin{array}{l}\text { CATGATCATAAGAGAAAA } \\
\text { CCTT }\end{array}$ \\
\hline CST3 & rs1064039 & TCTATCTAGCTCCAGCCTCTCG & САСАСССТССТССТССАС & GGGGCTCACGGCCAGGGCC & GACTGGAGCCGGㄷG \\
\hline
\end{tabular}


Tabelle 7: Hardy-Weinberg-Gleichgewicht der untersuchten Polymorphismen

\begin{tabular}{lr}
\hline Marker & Exakter p-Wert \\
\hline BIN1 & 1,0000 \\
IL8 & 0,5170 \\
CLU & 0,7240 \\
PICALM & 1,0000 \\
GWA14g32.13 & 0,7620 \\
ACE & 0,7380 \\
TNK1 & 0,0510 \\
LDLR & 0,7590 \\
ApoE234 & 0,5500 \\
EXOC3L2 & 1,0000 \\
CST3 & 0,6400 \\
\hline
\end{tabular}

\subsection{Durchführung}

Die Rekrutierung und die Untersuchung der Patienten fanden im Zeitraum von Juli 2008 bis August 2010 statt.

Nach Aufklärung und Einwilligung des Patienten bzw. dessen Angehörigen wurde eine Erstuntersuchung durchgeführt. Diese fand in den meisten Fällen in dem Krankenhaus statt, das den Patienten ursprünglich gemeldet hatte. In wenigen Fällen wurde der Patient in häuslicher Umgebung oder in einer Senioreneinrichtung besucht. Die Untersuchung umfasste eine ausführliche Anamnese und Fremdanamnese sowie eine gründliche körperliche Untersuchung, welche eine eingehende Prüfung des neurologischen Status beinhaltete. Zur Erfassung einer möglichen extrapyramidalen Symptomatik wurde zudem der UPDRS-IIIScore (Fahn et al. 1987) erhoben. Eine neuropsychologische Testung zur Prüfung der kognitiven Funktionen wurde mithilfe der CERAD-plus-Testbatterie durchgeführt. Des Weiteren kam der GDS-Score zum Einsatz, der anhand einer 7-Stadien-Einteilung eine Einschätzung der Schwere der kognitiven Defizite ermöglicht (Reisberg et al. 1988). Der $A D L$-Score wurde zur Beurteilung der Aktivitäten des täglichen Lebens verwendet (Lawton und Brody 1969). Zum Abschluss der Untersuchung erfolgte eine Akquirierung von Blutproben. Der Zeitumfang der gesamten Untersuchung betrug etwa zwei bis drei Stunden. 
Im Anschluss an den Patientenbesuch wurden innerhalb der folgenden 24 Stunden die Blutproben aufgearbeitet und eingefroren.

Sechs Monate nach der Erstuntersuchung erfolgte ein telefonisches Follow-up. Dieses umfasste eine telefonisch durchführbare Version des MMST (Newkirk et al. 2004) sowie eine erneute Erhebung des $G D S$ - und ADL-Scores mit Unterstützung naher Angehöriger des Patienten.

Die Zweituntersuchung, welche ein Jahr nach Studieneinschluss stattfand, entsprach in der Durchführung der Erstuntersuchung. Die Patienten wurden hier jedoch in ihrer häuslichen Umgebung oder gegebenenfalls einer Heimeinrichtung besucht.

\subsection{Erfassung und Auswertung der Daten}

\subsubsection{Dateneingabe und -verarbeitung}

Zur Eingabe der erhobenen und gemessenen Daten wurde das Programm Excel® 2007 der Firma Microsoft (Seattle, Washington, USA) verwendet. Die mit der CERAD-Testbatterie erfassten Rohdaten wurden mithilfe des von der Memory Clinic Basel entwickelten Add-ins CERAD-Plus 1.0 für Excel eingegeben, in demographisch angepasste z-Werte umgerechnet und mit einem Leistungsprofil grafisch dargestellt.

\subsubsection{Statistische Analysen}

Sämtliche statistische Analysen wurden mit dem Programm PASW Statistics ® 18.0.0 der Firma SPSS Inc. (Chicago, Illinois, USA) durchgeführt.

Bei der deskriptiven Statistik wurden kategoriale Daten in Prozentangaben ausgedrückt. Normalverteilte metrische Werte wurden als Mittelwert mit Standardabweichung (SD), nicht normalverteilte metrische Werte als Median mit Interquartilabstand (IQR) angegeben. Die Normalverteilung wurde stets mit dem Shapiro-Wilk-Test überprüft.

Bei der Untersuchung eines Zusammenhangs zwischen kategorialen Merkmalen wurde immer der exakte Test nach Fisher verwendet, um bei den geringen Gruppengrößen eine bestmögliche Aussagekraft zu erhalten. Bei Kontingenztafeln, die größer als 2x2 Felder waren, kam entsprechend ein in PASW implementierter erweiterter Fisher-Exakt-Test zum Einsatz. Für die Analyse von Zusammenhängen zwischen zwei metrischen Variablen wurde bei normalverteilten Daten der Korrelationskoeffizient nach Pearson und bei nicht 
normalverteilten Werten der Korrelationskoeffizient nach Spearman berechnet. Lag ein metrisches und ein kategoriales Merkmal vor, wurde zur Feststellung eines Unterschieds zwischen den Stufen des kategorialen Merkmals bei normalverteilten Werten der T-Test, bei nicht normalverteilten Werten der Mann-Whitney-Test angewandt. Bei mehr als 2 Stufen des kategorialen Merkmals kam bei normalverteilten Werten die einfaktorielle ANOVA, bei nicht normalverteilten Daten der Kruskal-Wallis-Test zum Einsatz. Bei allen statistischen Tests wurde eine Irrtumswahrscheinlichkeit hinsichtlich eines Fehlers 1. Art ( $\alpha$-Fehler) von p < 0,05 als statistisch signifikant angenommen. 


\section{Ergebnisse}

\subsection{Beschreibung des Patientenkollektivs}

Zum Zeitpunkt der Analyse bestand das Patientenkollektiv der rpAD-Studie aus 66 Teilnehmern. Für die Auswertung im Rahmen der vorliegenden Arbeit wurden jedoch nur Patienten berücksichtigt, bei denen eine Erst- und Zweituntersuchung und somit eine Berechnung der Krankheitsprogression anhand des MMST durchgeführt werden konnte. Somit bestand die weiter untersuchte Gruppe aus 42 Studienteilnehmern.

\subsubsection{Soziodemographische Daten}

\subsubsection{Alter und Geschlecht}

26 Patienten (62 \%) waren weiblichen, 16 Patienten (38\%) männlichen Geschlechts. Das Durchschnittsalter der Patienten bei Erstuntersuchung betrug 67 Jahre (SD = 9,6), die Altersspanne lag zwischen 49 und 86 Jahren. Die weiblichen Teilnehmer waren durchschnittlich 69 Jahre (SD = 9,9) alt, das Durchschnittsalter der Männer betrug 64 Jahre $(\mathrm{SD}=8,5)$. Der geschlechtsspezifische Altersunterschied war nicht statistisch signifikant (TTest: $p=0,086$, siehe Abb. 4). Eine Übersicht wird in Tabelle 8 dargestellt.

Tabelle 8: Alter der Patienten

\begin{tabular}{llll}
\hline Alter (in Jahren) & Frauen $(61,9 \%)$ & Männer $(38,1 \%)$ & gesamt \\
\hline Mittelwert & 69,01 & 63,77 & 67,01 \\
SD & 9,88 & 8,45 & 9,61 \\
Median & 71,69 & 63,24 & 67,31 \\
IQR & 10,85 & 14,32 & 14,77 \\
Minimum & 49,49 & 51,18 & 49,49 \\
Maximum & 86,14 & 82,33 & 86,14 \\
Spannweite & 36,65 & 31,15 & 36,65 \\
\hline
\end{tabular}




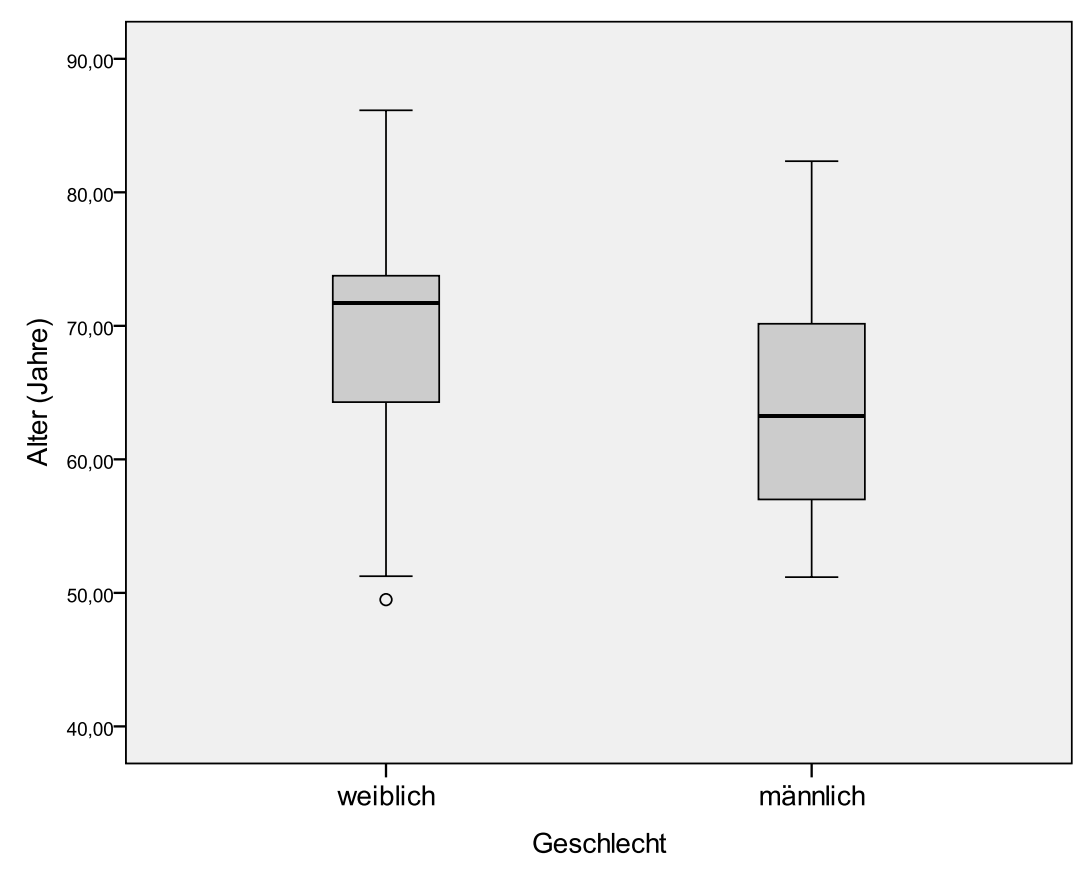

T-Test: $p=0,086$

Abb. 4: Geschlechtsspezifischer Altersunterschied im Patientenkollektiv

\subsubsection{Krankheitsbeginn und Krankheitsdauer}

Bei 17 Patienten (40,5\%) lag eine AD mit frühem Krankheitsbeginn (EOAD) vor, bei 25 (59,5\%) eine AD mit spätem Beginn (LOAD). Das Verhältnis zwischen frühem und spätem Beginn zeigte im Geschlechtervergleich einen statistisch signifikanten Unterschied, wobei Frauen tendenziell später erkrankten, dagegen in der EOAD-Gruppe mehr Männer waren (Exakter Test nach Fisher: $p=0,029$, Tabelle 9). Auch die Krankheitsdauer (Zeit vom Beginn der Symptomatik bis zur Erstuntersuchung) unterschied sich bei den Geschlechtern signifikant (Mann-Whitney-Test: $p=0,047$, Tabelle 10).

Tabelle 9: Krankheitsbeginn der Patienten

\begin{tabular}{|c|c|c|c|c|c|c|}
\hline & \multicolumn{2}{|c|}{ Frauen } & \multicolumn{2}{|c|}{ Männer } & \multicolumn{2}{|c|}{ Total } \\
\hline & EOAD & LOAD & EOAD & LOAD & EOAD & LOAD \\
\hline Anzahl & 7 & 19 & 10 & 6 & 17 & 25 \\
\hline$\%$ & 26,9 & 73,1 & 62,5 & 37,5 & 40,5 & 59,5 \\
\hline
\end{tabular}

EOAD: Krankheitsbeginn $\leq$ 65. Lebensjahr; LOAD: Krankheitsbeginn > 65. Lebensjahr Geschlechtsspezifischer Unterschied: $p=0,029$ (Exakter Test nach Fisher) 
Tabelle 10: Krankheitsdauer der Studienteilnehmer (in Monaten)

\begin{tabular}{llll}
\hline Krankheitsdauer & Frauen $(61,9 \%)$ & Männer $(38,1 \%)$ & Total \\
\hline Mittelwert & 18,75 & 30,21 & 23,12 \\
SD & 16,66 & 22,43 & 19,62 \\
Median & 14,66 & 30,04 & 16,90 \\
IQR & 25,49 & 40,89 & 28,36 \\
Minimum & 1,87 & 6,64 & 1,87 \\
Maximum & 72,95 & 74,27 & 74,27 \\
Spannweite & 71,08 & 67,63 & 72,40 \\
\hline
\end{tabular}

Krankheitsdauer vs. Geschlecht: $p=0,047$ (Mann-Whitney-Test)

\subsubsection{Kognitiver Status}

\subsubsection{MMST bei Erst- und Zweituntersuchung}

Der Median der MMST-Punktzahlen aller Studienteilnehmer bei Erstuntersuchung lag bei 21,5 Punkten (IQR = 9,0, siehe Tabelle 11 und Abb. 5). Die weiblichen Teilnehmer schnitten mit einem Median von 16,5 Punkten (IQR = 11,0) schlechter ab als die männlichen Patienten $(\tilde{x}=24,0$ Punkte, IQR $=5,5)$ ab. Diese Differenz erwies sich als statistisch signifikant (Mann-Whitney-Test: $p=0,011$, siehe Abb. 6).

Beim MMST der Zweituntersuchung betrug der Median 18,0 Punkte (IQR = 11,5, siehe Tabelle 11 und Abb. 5). Auch hier lag der Median bei den männlichen Patienten mit 20,0 Punkten $(\mathrm{IQR}=7,5)$ höher als bei den weiblichen Teilnehmern $(\tilde{x}=14,5$ Punkte, IQR $=$ 14,8). Diese Differenz erwies sich als statistisch nicht signifikant (Mann-Whitney-Test: $p=$ 0,173, siehe Abb. 7).

Tabelle 11: MMST-Punktzahlen bei Erst- (EU) und Zweituntersuchung (ZU)

\begin{tabular}{lccccccc}
\hline MMST & \multicolumn{2}{c}{ Frauen } & & \multicolumn{2}{c}{ Männer } & \multicolumn{2}{c}{ Total } \\
\cline { 2 - 3 } \cline { 7 - 8 } & MMST EU & MMST ZU & & MMST EU & MMST ZU & MMST EU & MMST ZU \\
\hline MW & 17,19 & 14,19 & & 22,69 & 18,00 & 19,29 & 15,64 \\
SD & 7,21 & 8,25 & & 4,06 & 6,69 & 6,71 & 7,83 \\
Median & 16,50 & 14,50 & & 24,00 & 20,00 & 21,50 & 18,00 \\
IQR & 11,00 & 14,75 & & 5,50 & 7,50 & 9,00 & 11,50 \\
\hline
\end{tabular}




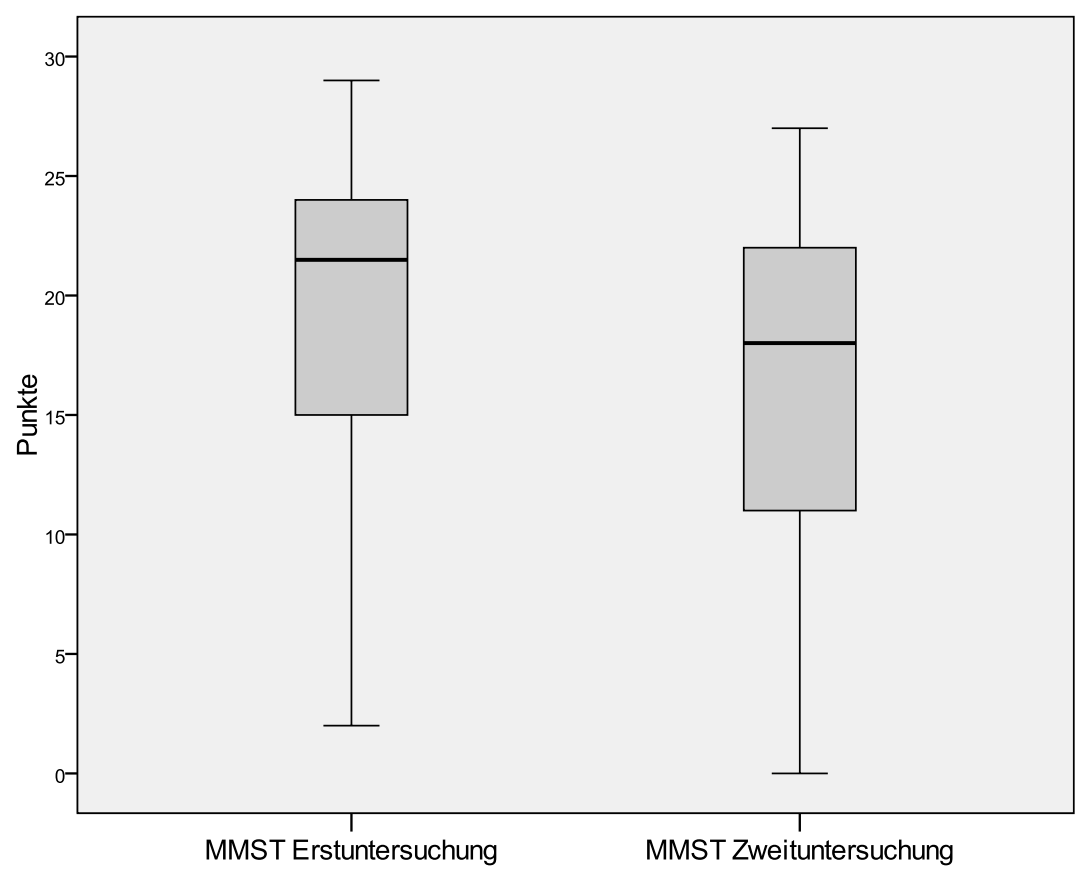

Abb. 5: MMST bei der Erst-und Zweituntersuchung

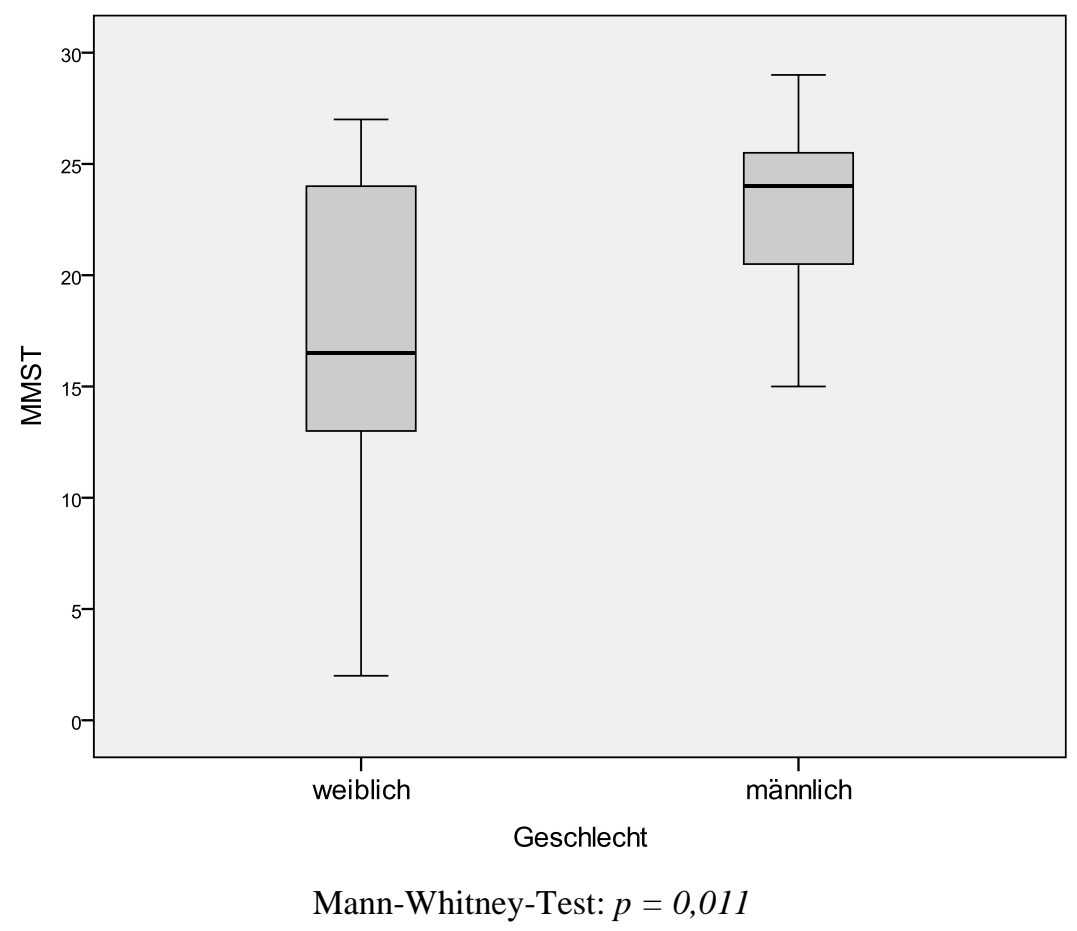

Abb. 6: Geschlechtsspezifische Unterschiede im MMST bei Erstuntersuchung 


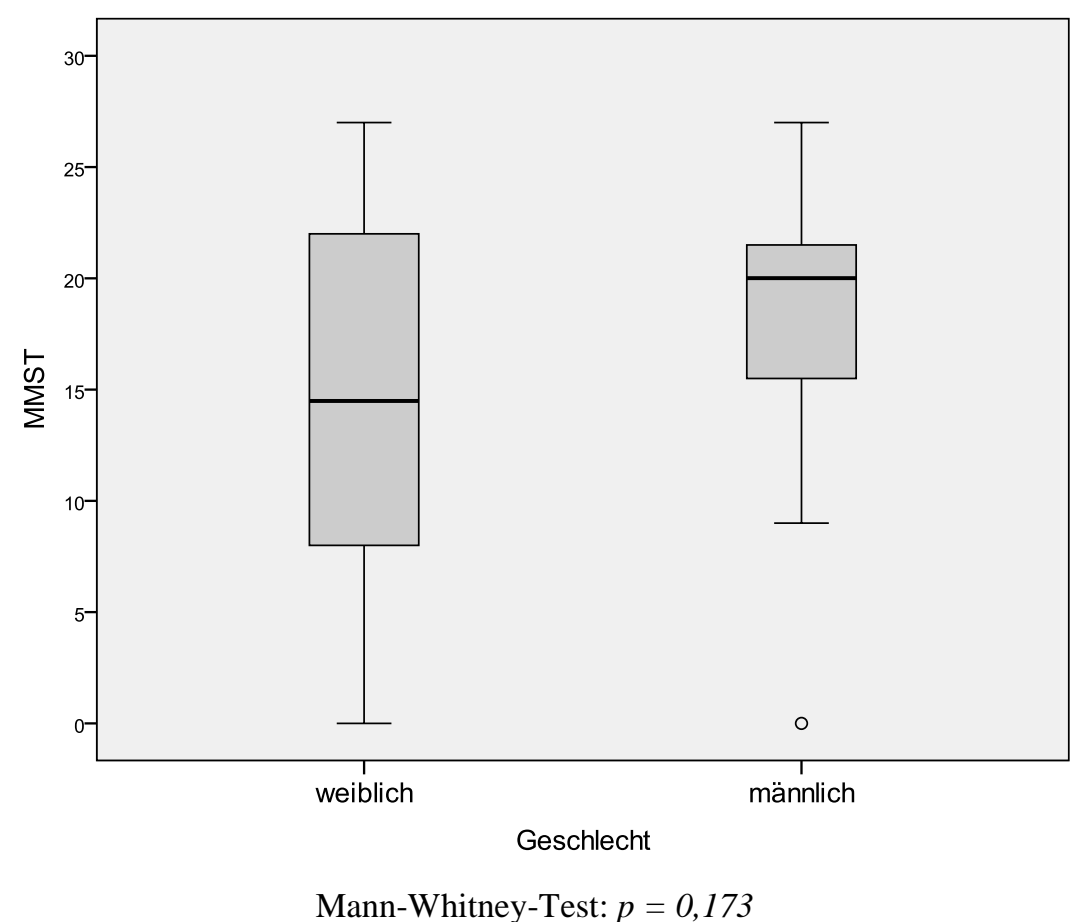

Abb. 7: Geschlechtsspezifische Unterschiede im MMST bei Zweituntersuchung

\subsubsection{MMST-Progression}

Die MMST-Progression wurde nach folgender Formel berechnet:

$$
\frac{\text { MMSTErstuntersuchung - MMSTZweituntersuchung }}{\text { Zeit zwischen Erst - und Zweituntersuchung in Jahren }}
$$

Der Median der MMST-Progression aller Patienten lag bei 3,7 Punkten/Jahr (IQR = 5,3), d.h. um diesen Zentralwert verschlechterten sich die Studienteilnehmer von der Erst- zur Zweituntersuchung. Die Leistungsspanne reichte von einer Verbesserung um 1,9 Punkte/Jahr bis zu einer Verschlechterung um 14,8 Punkte/Jahr. Beim geschlechtsspezifischen Vergleich der Progressionsraten fiel auf, dass die Frauen mit einem Median von 2,0 Punkten (IQR = 5,9) eine deutlich langsamere MMST-Progression als die Männer ( $\tilde{x}=4,0, \mathrm{IQR}=2,8)$ aufwiesen (siehe Tabelle 12). Diese Differenz war jedoch nicht statistisch signifikant (Mann-WhitneyTest: $p=0,084)$. Bei der Stratifikation nach Erkrankungsbeginn konnte keine deutliche Differenz hinsichtlich der MMST-Progression festgestellt werden (siehe Tabelle 12). Zudem zeigte sich kein Hinweis für eine Korrelation zwischen MMST-Progression und AusgangsMMST, Alter, Ausbildung oder Krankheitsdauer (siehe Tabelle 13). 
Tabelle 12: MMST-Progression in Abhängigkeit von Geschlecht und Krankheitsbeginn

\begin{tabular}{|c|c|c|c|c|c|c|c|c|c|}
\hline \multirow{2}{*}{$\begin{array}{c}\Delta \\
\text { MMST }\end{array}$} & \multicolumn{3}{|c|}{ Alle } & \multicolumn{3}{|c|}{ Frauen } & \multicolumn{3}{|c|}{ Männer } \\
\hline & Total & EOAD & LOAD & Total & EOAD & LOAD & Total & EOAD & LOAD \\
\hline Median & 3,69 & 2,93 & 3,73 & 2,03 & 2,90 & 1,77 & 3,98 & 3,45 & 4,41 \\
\hline IQR & 5,32 & 5,83 & 5,62 & 5,91 & 7,55 & 5,81 & 2,78 & 3,40 & 5,12 \\
\hline
\end{tabular}

$\triangle$ MMST: MMST-Progression

EOAD vs. LOAD: $p=0,985^{a}$

Frauen (EOAD vs. LOAD): $p=0,831^{a}$

${ }^{a}$ Mann-Whitney-Test

Frauen vs. Männer: $p=0,084^{a}$

Männer (EOAD vs. LOAD): $p=0,220^{a}$

Tabelle 13: Korrelation zwischen MMST-Progression und MMST, Alter, Ausbildung und Krankheitsdauer bei Erstuntersuchung

\begin{tabular}{lcccc}
\hline MMST-Progression & MMST $^{\mathbf{b}}$ & Alter $^{\mathbf{b}}$ & Ausbildung & Krankheitsdauer $^{\mathbf{b}}$ \\
\hline Korrelationskoeffizient $^{\mathbf{a}}$ & 0,067 & $-0,037$ & $-0,058$ & 0,145 \\
$\boldsymbol{P}$ & 0,672 & 0,818 & 0,717 & 0,361 \\
\hline
\end{tabular}

${ }^{\mathrm{a}}$ nach Spearman, ${ }^{\mathrm{b}}$ bei Erstuntersuchung

Die Studienpatienten wurden abhängig von ihrer MMST-Progression in zwei Kategorien eingeteilt:

1. „Slow“ (langsamer Verlauf): MMST-Progression $\leq 5$

2. „Rapid“ (schneller Verlauf): MMST-Progression > 5

Die o.g. Grenze wurde gewählt, da ein MMST-Verfall von über 5 Punkten/Jahr von einigen Autoren als Definitionskriterium der rpAD vorgeschlagen wurde (Doody et al. 2001; Wallin et al. 2010). Zum anderen entsprach dieser Wert ungefähr dem 75. Perzentil der MMSTProgression aller Patienten im Kollektiv. Die Aufteilung der Patienten auf die jeweiligen Kategorien sowie deren Charakterisierung zeigen die Tabellen 14 und 15. Weder zwischen Frauen und Männern noch zwischen Probanden mit frühem und spätem Krankheitsbeginn zeigten sich deutliche Unterschiede hinsichtlich der Verteilung auf die Kategorien slow und rapid. Auch hinsichtlich des Ausgangs-MMST, des Alters, der Ausbildung und der 
Krankheitsdauer konnten keine deutlichen Differenzen zwischen Patienten mit langsam und schnell definiertem Verlauf festgestellt werden.

Tabelle 14: Krankheitsverlauf in Abhängigkeit von Geschlecht und Krankheitsbeginn

\begin{tabular}{|c|c|c|c|c|c|c|}
\hline & & \multirow[t]{2}{*}{ Gesamt } & \multicolumn{2}{|c|}{ Geschlecht } & \multicolumn{2}{|c|}{ Krankheitsbeginn } \\
\hline & & & weiblich & männlich & EOAD & LOAD \\
\hline \multirow[t]{2}{*}{ Slow } & $\mathrm{n}$ & 32 & 20 & 12 & 13 & 19 \\
\hline & $\%$ & 76,2 & 76,9 & 75,0 & 76,5 & 76,0 \\
\hline \multirow[t]{2}{*}{ Rapid } & $\mathrm{n}$ & 10 & 6 & 4 & 4 & 6 \\
\hline & $\%$ & 23,8 & 23,1 & 25,0 & 23,5 & 24,0 \\
\hline
\end{tabular}

Krankheitsverlauf vs. Geschlecht: $p=1,000^{a}$

${ }^{\text {a }}$ Exakter Test nach Fisher

Krankheitsverlauf vs. -beginn: $p=1,000^{a}$

Tabelle 15: Krankheitsverlauf in Abhängigkeit von MMST, Alter, Ausbildung und Krankheitsdauer bei Erstuntersuchung

\begin{tabular}{lccccc}
\hline & & $\begin{array}{c}\text { MMST }^{\mathbf{c}} \\
(\text { Punkte })\end{array}$ & $\begin{array}{c}\text { Alter }^{\mathbf{c}} \\
(\text { Jahre })\end{array}$ & $\begin{array}{c}\text { Ausbildung } \\
(\text { Jahre })\end{array}$ & $\begin{array}{c}\text { Krankheitsdauer }^{\mathbf{c}} \\
\text { Slow }\end{array}$ \\
& MW & 19,44 & 67,05 & 11,25 & 22,99 \\
& SD & 6,95 & 9,40 & 3,13 & 20,31 \\
& Median & 22,50 & 67,09 & 11,00 & 14,66 \\
& IQR & 9,00 & 15,26 & 4,25 & 27,80 \\
\hline Rapid & MW & 18,80 & 66,90 & 10,10 & 23,53 \\
& SD & 6,21 & 10,78 & 3,31 & 18,24 \\
& Median & 18,50 & 69,25 & 8,00 & 27,91 \\
& IQR & 12,00 & 16,12 & 3,50 & 31,29 \\
\hline Slow vs. & $p$ & $0,699^{\mathrm{b}}$ & $0,968^{\mathrm{a}}$ & $0,238^{\mathrm{b}}$ & $0,925^{\mathrm{b}}$ \\
rapid & & & & \\
\hline
\end{tabular}

${ }^{\mathrm{a}}$ T-Test, ${ }^{\mathrm{b}}$ Mann-Whitney-Test, ${ }^{\mathrm{c}}$ bei Erstuntersuchung 


\subsection{Genetische Polymorphismen}

\subsubsection{Genotyp- und Allelverteilung im Gesamtkollektiv}

Die 11 Polymorphismen wurden hinsichtlich eines Zusammenhangs zwischen der Genotypbzw. Allelverteilung und der Krankheitsprogression der jeweiligen Träger untersucht. Tabelle 16 zeigt die Genotyp- und Allelverteilung innerhalb des Gesamtkollektivs sowie innerhalb der als langsam und schnell definierten Gruppen (,slow" vs. „rapid"). Zudem sind die Mittelwerte der MMST-Progression der jeweiligen Träger aufgeführt. Die Gene mit statistisch signifikanten bzw. Trend-Ergebnissen werden im Anschluss ausführlich dargestellt.

Tabelle 16: Untersuchte Polymorphismen: Genotyp- und Allelverteilung mit jeweiliger Progression

\begin{tabular}{|c|c|c|c|c|c|c|}
\hline${\operatorname{Apo~} E^{\dagger}}^{+}$ & $\varepsilon 2 / 4$ & $\varepsilon 3 / 3$ & $\varepsilon 3 / 4$ & $\varepsilon 4 / 4$ & & $\varepsilon 4$-Träger \\
\hline Total $(\mathrm{n}=41)$ & $1(2,4 \%)$ & $17(41,5 \%)$ & $18(43,9 \%)$ & $5(12,2 \%)$ & & $24(58,5 \%)$ \\
\hline Slow $(n=31)$ & $1(3,2 \%)$ & $12(38,7 \%)$ & $14(45,2 \%)$ & $4(12,9 \%)$ & & $19(61,3 \%)$ \\
\hline Rapid $(\mathrm{n}=10)$ & $0(0,0 \%)$ & $5(50,0 \%)$ & $4(40,0 \%)$ & $1(10,0 \%)$ & & $5(50,0 \%)$ \\
\hline $\mathrm{p}$-Wert* & \multicolumn{3}{|c|}{$0,909^{\mathrm{a}}$} & & & $0,714^{\mathrm{a}}$ \\
\hline$\Delta \mathrm{MMST} \bar{x}(\mathrm{SD})$ & 3,73 & $3,13(4,09)$ & $4,16(4,37)$ & $1,65(4,05)$ & & $3,62(4,25)$ \\
\hline$\Delta \mathrm{MMST} \tilde{x}(\mathrm{IQR})$ & 3,73 & $2,79(6,14)$ & $3,85(3,36)$ & $-0,89(7,42)$ & & $3,76(5,34)$ \\
\hline $\mathrm{p}$-Wert ${ }^{+}$ & \multicolumn{3}{|c|}{$0,743^{\mathrm{e}}$} & & & $0,671^{\mathrm{c}}$ \\
\hline$C L U$ & $\overline{\mathrm{CC}}$ & $\mathrm{CT}$ & TT & & C-Träger & T-Träger \\
\hline Total $(\mathrm{n}=40)$ & $17(42,5 \%)$ & $17(42,5 \%)$ & $6(15,0 \%)$ & & $34(85,0 \%)$ & $23(57,5 \%)$ \\
\hline Slow $(n=30)$ & $12(40,0 \%)$ & $14(46,7 \%)$ & $4(13,3 \%)$ & & $26(86,7 \%)$ & $18(60,0 \%)$ \\
\hline Rapid $(\mathrm{n}=10)$ & $5(50,0 \%)$ & $3(30,0 \%)$ & $2(20,0 \%)$ & & $8(80,0 \%)$ & $5(50,0 \%)$ \\
\hline p-Wert* & & $0,691^{\mathrm{a}}$ & & & $0,629^{\mathrm{a}}$ & $0,717^{\mathrm{a}}$ \\
\hline$\Delta \mathrm{MMST} \bar{x}(\mathrm{SD})$ & $3,06(3,34)$ & $3,51(4,49)$ & $4,51(5,81)$ & & $3,29(3,90)$ & $3,77(4,75)$ \\
\hline$\Delta \mathrm{MMST} \tilde{x}(\mathrm{IQR})$ & $2,93(7,17)$ & $3,71(5,53)$ & $4,31(8,31)$ & & $3,32(5,95)$ & $3,71(5,83)$ \\
\hline p-Wert ${ }^{+}$ & & $0,908^{\mathrm{e}}$ & & & $0,677^{\mathrm{c}}$ & $0,919^{c}$ \\
\hline PICALM & $\overline{\mathrm{CC}}$ & $\overline{\mathrm{TC}}$ & TT & & C-Träger & T-Träger \\
\hline Total $(\mathrm{n}=40)$ & $3(7,5 \%)$ & $16(40,0 \%)$ & $21(52,5 \%)$ & & $19(47,5 \%)$ & $37(92,5 \%)$ \\
\hline Slow $(n=30)$ & $2(6,7 \%)$ & $11(36,7 \%)$ & $17(56,7 \%)$ & & $13(43,3 \%)$ & $28(93,3 \%)$ \\
\hline Rapid $(\mathrm{n}=10)$ & $1(10,0 \%)$ & $5(50,0 \%)$ & $4(40,0 \%)$ & & $6(60,0 \%)$ & $9(90,0 \%)$ \\
\hline p-Wert* & & $0,540^{\mathrm{a}}$ & & & $0,473^{\mathrm{a}}$ & $1,000^{\mathrm{a}}$ \\
\hline$\Delta \mathrm{MMST} \bar{x}(\mathrm{SD})$ & $5,42(8,23)$ & $4,79(4,39)$ & $2,19(3,01)$ & & $4,89(4,86)$ & $3,31(3,84)$ \\
\hline$\Delta \mathrm{MMST} \tilde{x}(\mathrm{IQR})$ & 3,73 & $4,19(5,53)$ & $2,69(5,36)$ & & $3,97(6,07)$ & $3,66(5,67)$ \\
\hline $\mathrm{p}-$ Wert $^{+}$ & & $0,119^{\mathrm{d}}$ & & & $0,039^{\mathrm{b}}$ & $0,916^{\mathrm{c}}$ \\
\hline
\end{tabular}


Tabelle 16 (Fortsetzung)

\begin{tabular}{|c|c|c|c|c|c|}
\hline BIN1 & AA & $\mathrm{AG}$ & GG & A-Träger & G-Träger \\
\hline Total $(n=40)$ & $19(47,5 \%)$ & $17(42,5 \%)$ & $4(10,0 \%)$ & $36(90,0 \%)$ & $21(52,5 \%)$ \\
\hline Slow $(n=30)$ & $12(40,0 \%)$ & $16(53,3 \%)$ & $2(6,7 \%)$ & $28(93,3 \%)$ & $18(60,0 \%)$ \\
\hline Rapid $(n=10)$ & $7(70,0 \%)$ & $1(10,0 \%)$ & $2(20,0 \%)$ & $8(80,0 \%)$ & $3(30,0 \%)$ \\
\hline p-Wert* & \multicolumn{3}{|c|}{$0,037^{\mathrm{a}}$} & $0,256^{\mathrm{a}}$ & $0,148^{\mathrm{a}}$ \\
\hline$\Delta \mathrm{MMST} \bar{x}(\mathrm{SD})$ & $4,21(5,36)$ & $2,15(2,38)$ & $5,57(2,16)$ & $3,24(4,30)$ & $2,80(2,67)$ \\
\hline$\Delta \mathrm{MMST} \tilde{x}(\mathrm{IQR})$ & $2,93(7,91)$ & $3,66(4,41)$ & $5,79(4,15)$ & $3,30(5,82)$ & $3,71(4,62)$ \\
\hline $\mathrm{p}-\mathrm{Wert}{ }^{+}$ & \multicolumn{3}{|c|}{$0,191^{\mathrm{e}}$} & $0,114^{\mathrm{c}}$ & $0,763^{\mathrm{c}}$ \\
\hline$I L-8$ & AA & TA & TT & A-Träger & T-Träger \\
\hline Total $(n=40)$ & $4(10,0 \%)$ & $21(52,5 \%)$ & $15(37,5 \%)$ & $25(62,5 \%)$ & $36(90,0 \%)$ \\
\hline Slow $(n=30)$ & $3(10,0 \%)$ & $16(53,3 \%)$ & $11(36,7 \%)$ & $19(63,3 \%)$ & $27(90,0 \%)$ \\
\hline Rapid $(n=10)$ & $1(10,0 \%)$ & $5(50,0 \%)$ & $4(40,0 \%)$ & $6(60,0 \%)$ & $9(90,0 \%)$ \\
\hline $\mathrm{p}$-Wert* & \multicolumn{3}{|c|}{$1,000^{\mathrm{a}}$} & $1,000^{\mathrm{a}}$ & $1,000^{\mathrm{a}}$ \\
\hline$\Delta \mathrm{MMST} \bar{x}(\mathrm{SD})$ & $5,04(6,81)$ & $3,53(4,37)$ & $2,96(3,22)$ & $3,77(4,69)$ & $3,29(3,89)$ \\
\hline$\Delta \mathrm{MMST} \tilde{x}(\mathrm{IQR})$ & $3,13(12,19)$ & $3,71(5,87)$ & $3,66(6,38)$ & $3,71(5,84)$ & $3,69(6,03)$ \\
\hline p-Wert ${ }^{+}$ & \multicolumn{3}{|c|}{$0,684^{\mathrm{d}}$} & $0,929^{\mathrm{c}}$ & $0,738^{\mathrm{c}}$ \\
\hline GWA14q32.13 & AA & AT & TT & A-Träger & T-Träger \\
\hline Total $(n=40)$ & $8(20,0 \%)$ & $18(45,0 \%)$ & $14(35,0 \%)$ & $26(65,0 \%)$ & $32(80,0 \%)$ \\
\hline Slow $(n=30)$ & $5(16,7 \%)$ & $13(43,3 \%)$ & $12(40,0 \%)$ & $18(60,0 \%)$ & $25(83,3 \%)$ \\
\hline Rapid $(n=10)$ & $3(30,0 \%)$ & $5(50,0 \%)$ & $2(20 \%)$ & $8(80,0 \%)$ & $7(70,0 \%)$ \\
\hline p-Wert* & \multicolumn{3}{|c|}{$0,356^{\mathrm{a}}$} & $0,446^{\mathrm{a}}$ & $0,388^{\mathrm{a}}$ \\
\hline$\Delta \mathrm{MMST} \bar{x}(\mathrm{SD})$ & $5,18(6,48)$ & $3,17(3,67)$ & $2,87(3,12)$ & $3,79(4,67)$ & $3,04(3,39)$ \\
\hline$\Delta \mathrm{MMST} \tilde{x}(\mathrm{IQR})$ & $3,75(13,40)$ & $2,92(6,50)$ & $3,93(5,08)$ & $3,30(6,95)$ & $3,30(5,58)$ \\
\hline $\mathrm{p}-\mathrm{Wert}^{+}$ & \multicolumn{3}{|c|}{$0,433^{\mathrm{d}}$} & $0,905^{\mathrm{c}}$ & $0,697^{\mathrm{c}}$ \\
\hline$A C E$ & $\mathrm{CC}$ & $\mathrm{TC}$ & TT & C-Träger & T-Träger \\
\hline Total $(n=40)$ & $7(17,5 \%)$ & $22(55,0 \%)$ & $11(27,5 \%)$ & $29(72,5 \%)$ & $33(82,5 \%)$ \\
\hline Slow $(n=30)$ & $6(20,0 \%)$ & $15(50,0 \%)$ & $9(30,0 \%)$ & $21(70,0 \%)$ & $24(80,0 \%)$ \\
\hline Rapid $(n=10)$ & $1(10,0 \%)$ & $7(70,0 \%)$ & $2(20,0 \%)$ & $8(80,0 \%)$ & $9(90,0 \%)$ \\
\hline p-Wert* & \multicolumn{3}{|c|}{$0,689^{\mathrm{a}}$} & $0,696^{\mathrm{a}}$ & $0,656^{\mathrm{a}}$ \\
\hline$\Delta \mathrm{MMST} \bar{x}(\mathrm{SD})$ & $2,39(2,99)$ & $4,61(4,58)$ & $1,87(3,47)$ & $4,07(4,31)$ & $3,70(4,39)$ \\
\hline$\Delta \mathrm{MMST} \tilde{x}(\mathrm{IQR})$ & $3,66(5,31)$ & $3,85(4,68)$ & $2,79(5,74)$ & $3,73(5,19)$ & $3,71(6,10)$ \\
\hline p-Wert ${ }^{+}$ & \multicolumn{3}{|c|}{$0,243^{\mathrm{e}}$} & $0,154^{\mathrm{c}}$ & $0,682^{\mathrm{c}}$ \\
\hline TNK1 & AA & AT & TT & A-Träger & T-Träger \\
\hline Total $(n=40)$ & $5(12,5 \%)$ & $27(67,5 \%)$ & $8(20,0 \%)$ & $32(80,0 \%)$ & $35(87,5 \%)$ \\
\hline Slow $(n=30)$ & $4(13,3 \%)$ & $18(60,0 \%)$ & $8(26,7 \%)$ & $22(73,3 \%)$ & $26(86,7 \%)$ \\
\hline Rapid $(n=10)$ & $1(10,0 \%)$ & $9(90,0 \%)$ & $0(0,0 \%)$ & $\begin{array}{c}10 \\
(100,0 \%)\end{array}$ & $9(90,0 \%)$ \\
\hline $\mathrm{p}$-Wert* & & $0,178^{\mathrm{a}}$ & & $0,165^{\mathrm{a}}$ & $1,000^{\mathrm{a}}$ \\
\hline$\Delta \mathrm{MMST} \bar{x}(\mathrm{SD})$ & $1,09(3,70)$ & $4,41(4,34)$ & $1,76(2,91)$ & $3,89(4,37)$ & $3,81(4,17)$ \\
\hline$\Delta \mathrm{MMST} \tilde{x}(\mathrm{IQR})$ & $-1,00(5,26)$ & $3,90(4,91)$ & $2,61(6,19)$ & $3,72(6,47)$ & $3,73(5,51)$ \\
\hline $\mathrm{p}-\mathrm{Wert}^{+}$ & & $0,086^{\mathrm{e}}$ & & $0,225^{\mathrm{c}}$ & $0,121^{\mathrm{c}}$ \\
\hline
\end{tabular}


Tabelle 16 (Fortsetzung)

\begin{tabular}{|c|c|c|c|c|c|}
\hline LDLR & $\overline{\mathrm{AA}}$ & $\overline{\mathrm{GA}}$ & $\overline{\mathrm{GG}}$ & A-Träger & G-Träger \\
\hline Total $(\mathrm{n}=40)$ & $6(15,0 \%)$ & $18(45,0 \%)$ & $16(40,0 \%)$ & $24(60,0 \%)$ & $34(85,0 \%)$ \\
\hline Slow $(n=30)$ & $5(16,7 \%)$ & $14(46,7 \%)$ & $11(36,7 \%)$ & $19(63,3 \%)$ & $25(83,3 \%)$ \\
\hline Rapid $(\mathrm{n}=10)$ & $1(10,0 \%)$ & $4(40,0 \%)$ & $5(50,0 \%)$ & $5(50,0 \%)$ & $9(90,0 \%)$ \\
\hline $\mathrm{p}$-Wert* & & $0,791^{\mathrm{a}}$ & & $0,482^{\mathrm{a}}$ & $1,000^{\mathrm{a}}$ \\
\hline$\Delta \mathrm{MMST} \bar{x}(\mathrm{SD})$ & $3,13(6,31)$ & $3,40(3,97)$ & $3,68(3,75)$ & $3,33(4,51)$ & $3,53(3,81)$ \\
\hline$\Delta \mathrm{MMST} \tilde{x}(\mathrm{IQR})$ & $0,97(8,66)$ & $3,75(5,51)$ & $3,70(5,30)$ & $3,32(5,87)$ & $3,72(5,59)$ \\
\hline p-Wert ${ }^{+}$ & & $0,960^{\mathrm{d}}$ & & $0,628^{\mathrm{c}}$ & $0,399^{c}$ \\
\hline EXOC3L2 & $\mathrm{CC}$ & $\mathrm{TC}$ & $\mathrm{TT}$ & C-Träger & T-Träger \\
\hline Total $(\mathrm{n}=40)$ & $0(0,0 \%)$ & $11(27,5 \%)$ & $29(72,5 \%)$ & $11(27,5 \%)$ & $40(100,0 \%)$ \\
\hline Slow $(\mathrm{n}=30)$ & $0(0,0 \%)$ & $8(26,7 \%)$ & $22(73,3 \%)$ & $8(26,7 \%)$ & $30(100,0 \%)$ \\
\hline Rapid $(\mathrm{n}=10)$ & $0(0,0 \%)$ & $3(30,0 \%)$ & $7(70,0 \%)$ & $3(30,0 \%)$ & $10(100,0 \%)$ \\
\hline p-Wert* & - & \multicolumn{2}{|c|}{$1,000^{\mathrm{a}}$} & $1,000^{\mathrm{a}}$ & - \\
\hline$\Delta \mathrm{MMST} \bar{x}(\mathrm{SD})$ & - & $4,88(4,01)$ & $2,93(4,18)$ & $4,88(4,01)$ & - \\
\hline$\Delta \mathrm{MMST} \tilde{x}(\mathrm{IQR})$ & - & $3,90(3,29)$ & $2,79(6,16)$ & $3,90(3,29)$ & - \\
\hline p-Wert ${ }^{+}$ & - & \multicolumn{2}{|c|}{$0,133^{\mathrm{c}}$} & $0,133^{\mathrm{c}}$ & - \\
\hline$\overline{C S T 3}$ & $\overline{\mathrm{CC}}$ & $\overline{\mathrm{CT}}$ & TT & C-Träger & T-Träger \\
\hline Total $(n=40)$ & $24(60,0 \%)$ & $13(32,5 \%)$ & $3(7,5 \%)$ & $37(92,5 \%)$ & $16(40,0 \%)$ \\
\hline Slow $(\mathrm{n}=30)$ & $17(56,7 \%)$ & $10(33,3 \%)$ & $3(10,0 \%)$ & $27(90,0 \%)$ & $13(43,3 \%)$ \\
\hline Rapid $(n=10)$ & $7(70,0 \%)$ & $3(30,0 \%)$ & $0(0,0 \%)$ & $\begin{array}{c}10 \\
(100,0 \%)\end{array}$ & $3(30,0 \%)$ \\
\hline p-Wert* & & $0,864^{\mathrm{a}}$ & & $0,560^{\mathrm{a}}$ & $0,711^{\mathrm{a}}$ \\
\hline$\Delta \mathrm{MMST} \bar{x}(\mathrm{SD})$ & $3,50(4,34)$ & $3,56(4,40)$ & $2,85(2,53)$ & $3,52(4,30)$ & $3,43(4,06)$ \\
\hline$\Delta \mathrm{MMST} \tilde{x}(\mathrm{IQR})$ & $3,30(6,48)$ & $3,71(6,80)$ & 3,73 & $3,66(6,55)$ & $3,72(5,48)$ \\
\hline p-Wert ${ }^{+}$ & & $0,990^{\mathrm{e}}$ & & $0,993^{\mathrm{c}}$ & $0,897^{\mathrm{c}}$ \\
\hline
\end{tabular}

$\triangle$ MMST: Punktverlust im MMST/Jahr (MMST-Progression)

Slow: $\triangle \mathrm{MMST} \leq 5$

Rapid: $\triangle \mathrm{MMST}>5$

- keine Apo E ع2/2- oder $\varepsilon 2 / 3$-Träger im Kollektiv

* slow/rapid vs. Genotypen bzw. slow/rapid vs. Allelträger/Nicht-Allelträger

${ }^{+}$MMST-Progression vs. Genotypen bzw. MMST-Progression vs. Allelträger/Nicht-Allelträger

${ }^{a}$ Exakter Test nach Fisher

${ }^{\mathrm{b}}$ T-Test

${ }^{\mathrm{c}}$ Mann-Whitney-Test

${ }^{\mathrm{d}}$ Einfaktorielle ANOVA

${ }^{\mathrm{e}}$ Kruskal-Wallis-Test 


\subsubsection{PICALM}

$\underline{\text { Vergleich der Kategorien „slow“ und „rapid““ }}$

Die als langsam und schnell verlaufend definierten Patienten (slow vs. rapid) zeigten in der Verteilung der Genotypen CC, TC und TT keinen statistisch signifikanten Unterschied. Auch C-Träger und Nicht-C-Träger bzw. T-Träger und Nicht-T-Träger unterschieden sich nicht deutlich in Bezug auf ihre Zuordnung zu den Kategorien slow und rapid (siehe Tabelle 16).

\section{$\underline{\text { Vergleich der MMST-Progression }}$}

Der Vergleich der einzelnen Genotypen CC, TC und TT untereinander ergab hinsichtlich der durchschnittlichen MMST-Progression der jeweiligen Patienten zwar keinen statistisch signifikanten Unterschied, legte aber einen Trend $\mathrm{zu}$ einer schnelleren Progression bei steigender Anzahl von C-Allelen nahe ( $p=0,119$, siehe Abb. 8 und Tabelle 16). Im direkten Vergleich zwischen C- und Nicht-C-Trägern ließ sich mit einem Mittelwert von 4,9 Punkten (SD 4,9) bei C-Trägern ebenfalls eine höhere durchschnittliche MMST-Progression als bei Nicht-C-Trägern nachweisen ( $\bar{x}=2,2$ Punkte, SD 3,0). Dieser Unterschied erwies sich als statistisch signifikant ( $p=0,039$, siehe Abb. 9). T-Träger und Nicht-T-Träger unterschieden sich nicht signifikant in ihrer durchschnittlichen MMST-Progression (siehe Tabelle 16).

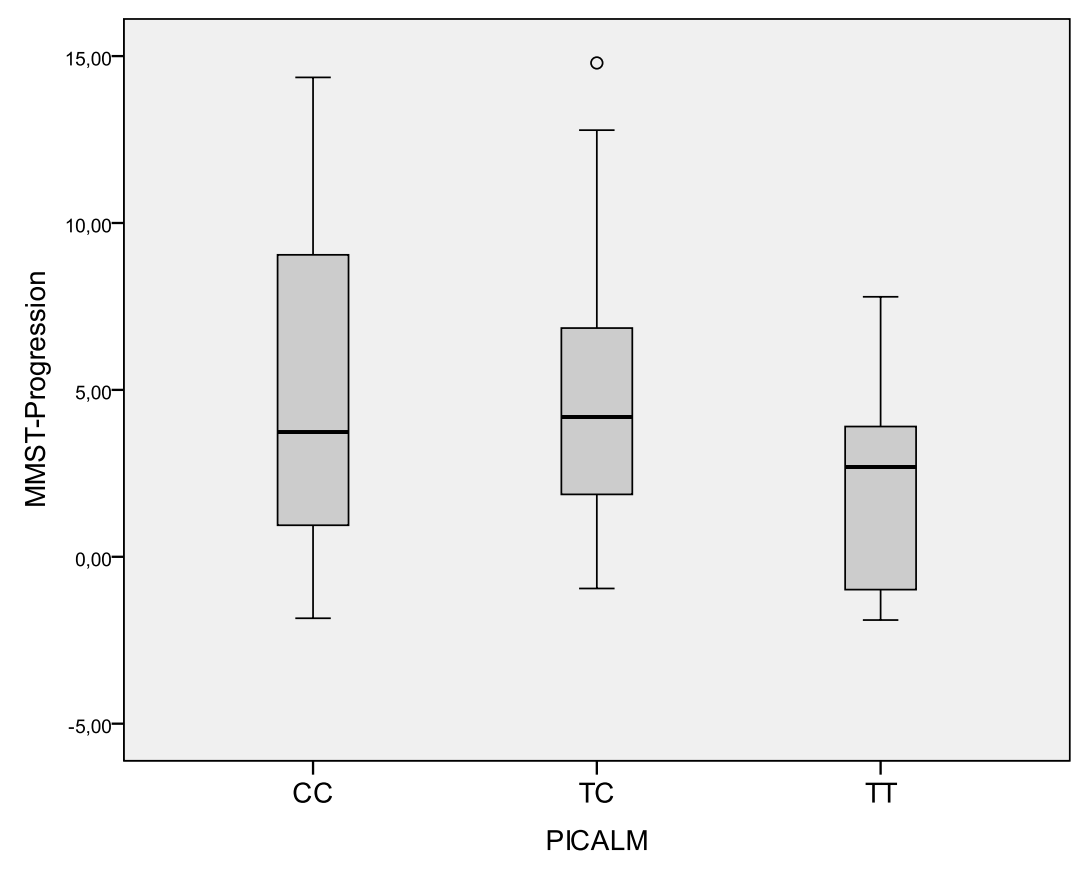

Einfaktorielle ANOVA: $p=0,119$

Abb. 8: MMST-Progression der PICALM-Genotypen 


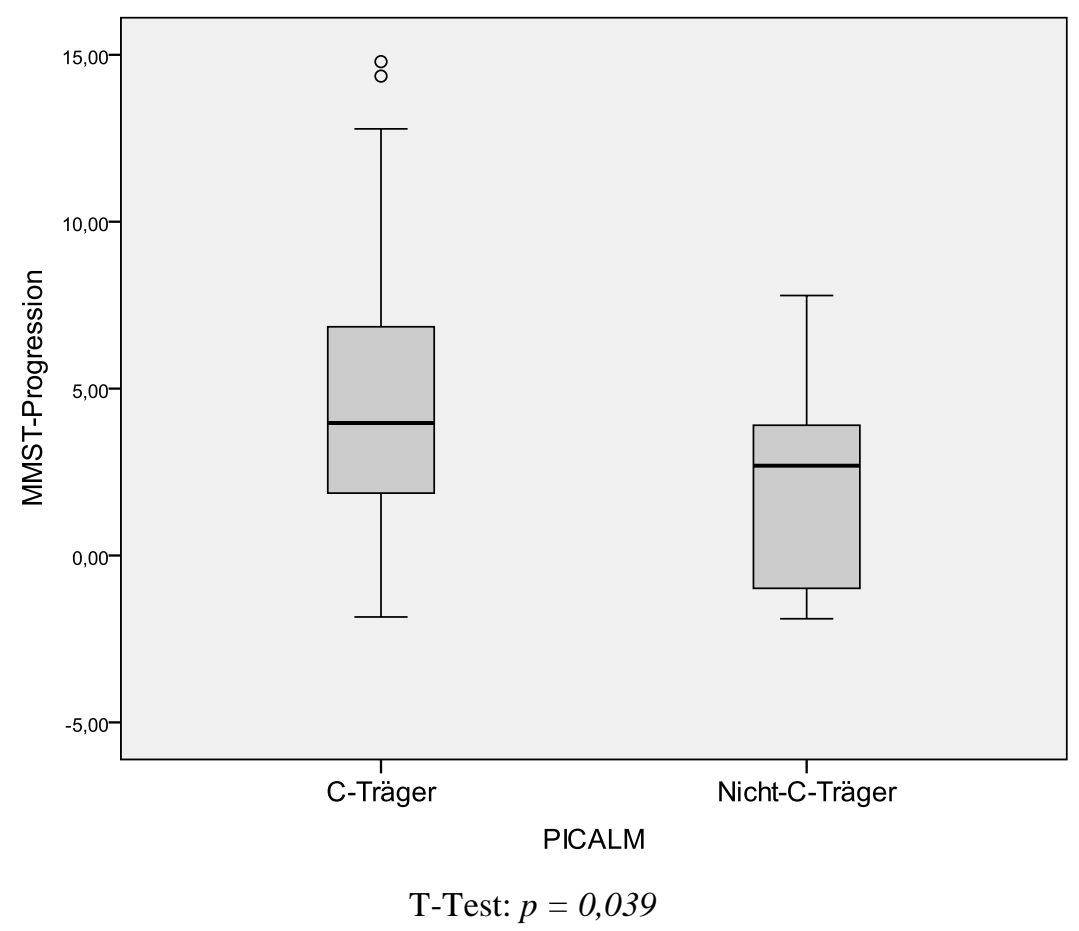

Abb. 9: PICALM: MMST-Progression von C-Trägern und Nicht-C-Trägern

\subsubsection{BIN1}

\section{$\underline{\text { Vergleich der Kategorien „slow“" und „rapid“" }}$}

Die Verteilung der Genotypen AA, AG und GG in den Gruppen slow und rapid war signifikant unterschiedlich ( $p=0,037$, siehe Abb. 10). Der Genotyp AG war im Vergleich zu den Genotypen AA/GG deutlich häufiger in der Gruppe der langsam verlaufenden Individuen anzutreffen $(p=0,026)$. Zwischen A-Trägern und Nicht-A-Trägern sowie zwischen GTrägern und Nicht-G-Trägern ließ sich hinsichtlich ihrer Zuordnung zu den Kategorien slow und rapid jedoch kein statistisch signifikanter Unterschied feststellen (siehe Tabelle 16).

\section{$\underline{\text { Vergleich der MMST-Progression }}$}

Die Träger der einzelnen Genotypen AA, AG und GG unterschieden sich untereinander nicht signifikant in ihrer jeweiligen MMST-Progression $(p=0,191)$, jedoch zeigte sich bei Studienteilnehmern ohne A-Allel (Genotyp GG) eine deutlich höhere MMST-Progression (siehe Abb. 11 und Tabelle 16). Letzteres konnte auch beim direkten Vergleich von ATrägern ( $\tilde{x}=3,3$ Punkte, IQR 5,8) und Nicht-A-Trägern ( $\tilde{x}=5,8$ Punkte, IQR 4,2) festgestellt werden, obgleich sich dieser Unterschied ebenfalls als nicht statistisch signifikant 
darstellte ( $p=0,114$, siehe Abb. 12). Zwischen G-Trägern und Nicht-G-Trägern konnte kein deutlicher Unterschied festgestellt werden (siehe Tabelle 16).

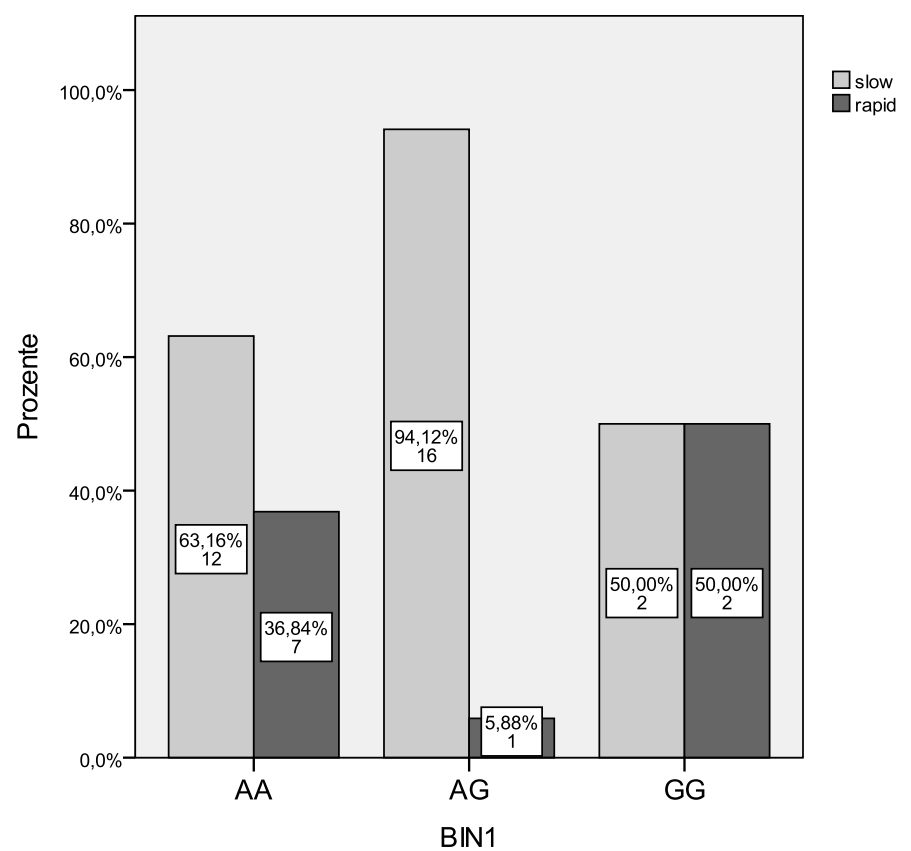

Exakter Test nach Fisher: AA vs. AG vs. GG: $p=0,037$, AG vs. AA/GG: $p=0,026$

Abb. 10: BIN1-Genotypen und Krankheitsverlauf („slow“ vs. „rapid“)

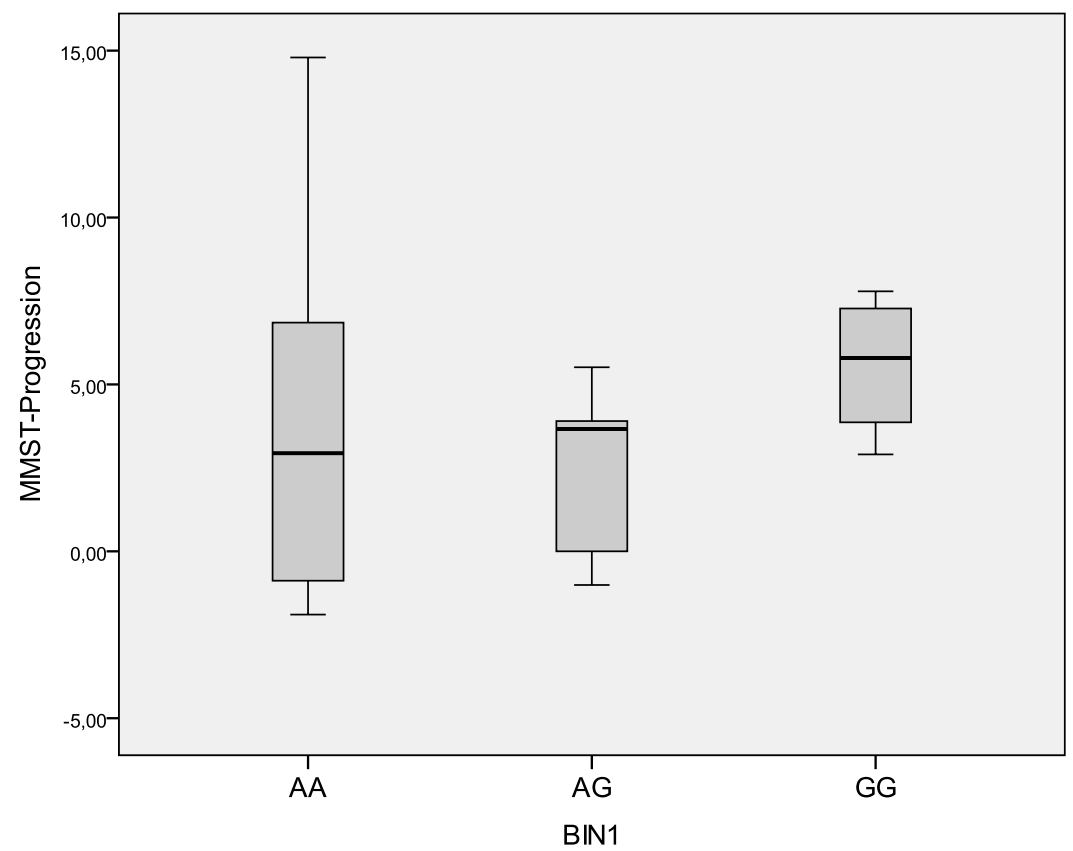

Kruskal-Wallis-Test: $p=0,191$

Abb. 11: MMST-Progression der BIN1-Genotypen 


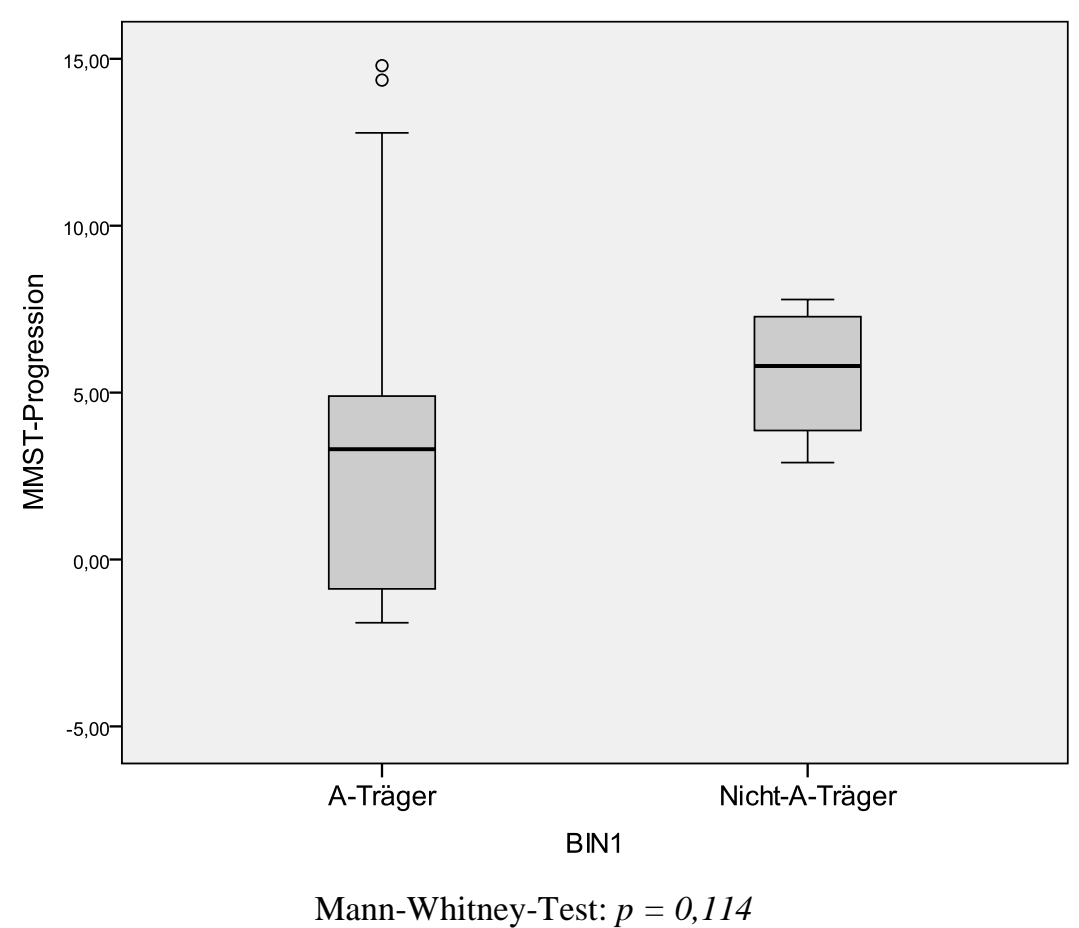

Abb. 12: BIN1: MMST-Progression von A-Trägern und Nicht-A-Trägern

\subsubsection{TNK1}

\section{$\underline{\text { Vergleich der Kategorien „slow“ und „rapid“ }}$}

Langsam und schnell verlaufende Fälle unterschieden sich nicht signifikant in der Verteilung der Genotypen AA, AT und TT. Auch der Vergleich der beiden Kategorien hinsichtlich der Häufigkeit von A-Trägern und Nicht-A-Trägern sowie von T-Trägern und Nicht-T-Trägern wies keine statistische Signifikanz auf (siehe Tabelle 16).

\section{$\underline{\text { Vergleich der MMST-Progression }}$}

Hinsichtlich der mittleren MMST-Progression der verschiedenen Genotyp-Träger konnte ein deutlicher, wenn auch nicht statistisch signifikanter Unterschied $(p=0,086)$ beobachtet werden. Während der Genotyp AT den schnellsten Verlauf zeigte, wies der Genotyp AA mit Abstand den langsamsten MMST-Verfall auf (siehe Abb. 13 und Tabelle 16). T-Träger ( $\tilde{x}=$ 3,7 Punkte, IQR 5,5) zeigten eine deutlich höhere Progression als Nicht-T-Träger ( $\tilde{x}=-1,0$ Punkte, IQR 5,3), wobei sich auch dieser Unterschied nicht als statistisch signifikant darstellte (siehe Abb. 14). A-Träger und Nicht-A-Träger unterschieden sich hinsichtlich der jeweiligen MMST-Progression nicht deutlich voneinander (siehe Tabelle 16). 


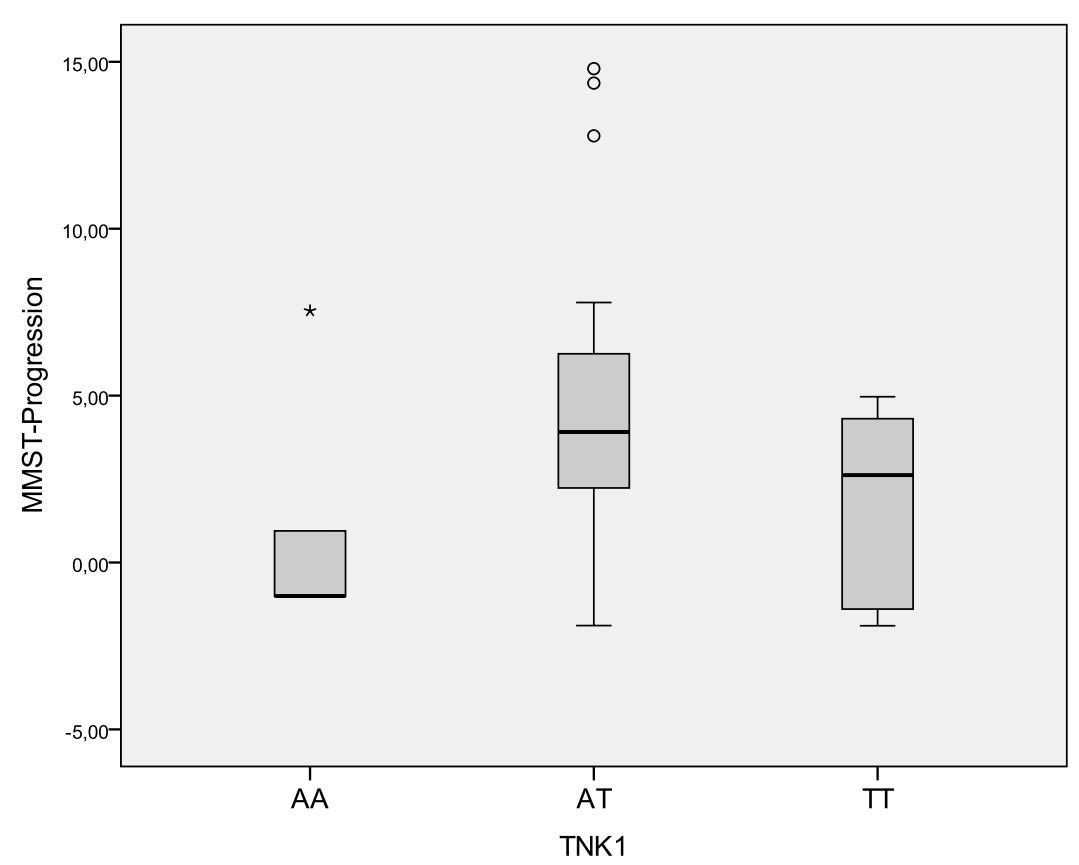

Kruskal-Wallis-Test: $p=0,086$

Abb. 13: MMST-Progression der TNK1-Genotypen

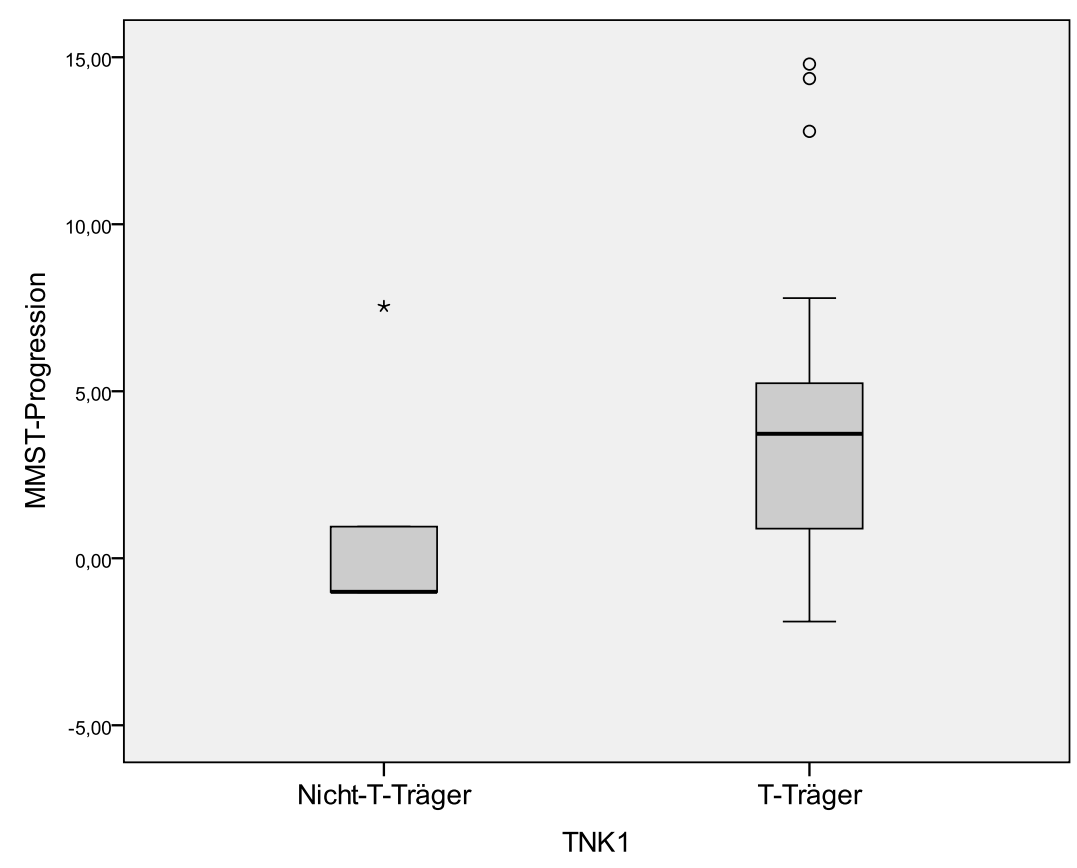

Mann-Whitney-Test: $p=0,121$

Abb. 14: TNK1: MMST-Progression der T-Träger und Nicht-T-Träger 


\subsubsection{Verteilung der Allelträger bei Frauen und Männern}

Tabelle 17 zeigt jeweils für Frauen und Männer eine Übersicht der Allelträger-Verteilung sowie der jeweiligen Krankheitsprogression. Signifikante bzw. Trend-Ergebnisse werden im Anschluss ausführlich beschrieben.

Tabelle 17: Untersuchte Polymorphismen nach Geschlecht stratifiziert: Allelverteilung und jeweilige Progression

\begin{tabular}{|c|c|c|}
\hline \multirow[t]{2}{*}{ Apo E } & \multicolumn{2}{|c|}{ Frauen } \\
\hline & $\varepsilon 4$-Träger & \\
\hline Total $(n=26)$ & $16(61,5 \%)$ & \\
\hline Slow $(n=20)$ & $13(65,0 \%)$ & \\
\hline Rapid $(n=6)$ & $3(50,0 \%)$ & \\
\hline p-Wert* & $0,644^{\mathrm{a}}$ & \\
\hline$\Delta \mathrm{MMST} \bar{x}(\mathrm{SD})$ & $2,63(4,15)$ & \\
\hline$\Delta \mathrm{MMST} \tilde{x}(\mathrm{IQR})$ & $3,00(5,57)$ & \\
\hline p-Wert ${ }^{+}$ & $0,846^{\mathrm{c}}$ & \\
\hline$C L U$ & C-Träger & T-Träger \\
\hline Total $(n=25)$ & $22(88,0 \%)$ & $13(52,0 \%)$ \\
\hline Slow $(\mathrm{n}=19)$ & $17(89,5 \%)$ & $10(52,6 \%)$ \\
\hline Rapid $(n=6)$ & $5(83,3 \%)$ & $3(50,0 \%)$ \\
\hline p-Wert* & $1,000^{\mathrm{a}}$ & $1,000^{\mathrm{a}}$ \\
\hline$\Delta \mathrm{MMST} \bar{x}(\mathrm{SD})$ & $3,08(4,48)$ & $3,06(5,30)$ \\
\hline$\Delta \mathrm{MMST} \tilde{x}(\mathrm{IQR})$ & $2,60(6,21)$ & $2,29(5,70)$ \\
\hline $\mathrm{p}-\mathrm{Wert}^{+}$ & $0,557^{\mathrm{c}}$ & $0,863^{\mathrm{c}}$ \\
\hline PICALM & C-Träger & T-Träger \\
\hline Total $(n=25)$ & $10(40,0 \%)$ & $22(88,0 \%)$ \\
\hline Slow $(n=19)$ & $7(36,8 \%)$ & $17(89,5 \%)$ \\
\hline Rapid $(n=6)$ & $3(50,0 \%)$ & $5(83,3 \%)$ \\
\hline p-Wert* & $0,653^{\mathrm{a}}$ & $1,000^{\mathrm{a}}$ \\
\hline$\Delta \mathrm{MMST} \bar{x}(\mathrm{SD})$ & $4,44(5,51)$ & $2,51(3,80)$ \\
\hline$\Delta \mathrm{MMST} \tilde{x}(\mathrm{IQR})$ & $3,76(8,43)$ & $2,03(5,91)$ \\
\hline $\mathrm{p}-\mathrm{Wert}^{+}$ & $0,143^{\mathrm{b}}$ & $0,716^{\mathrm{c}}$ \\
\hline BIN1 & A-Träger & G-Träger \\
\hline Total $(n=25)$ & $21(84,0 \%)$ & $15(60,0 \%)$ \\
\hline Slow $(n=19)$ & $17(89,5 \%)$ & $12(63,2 \%)$ \\
\hline Rapid $(n=6)$ & $4(66,7 \%)$ & $3(50,0 \%)$ \\
\hline p-Wert* & $0,234^{\mathrm{a}}$ & $0,653^{\mathrm{a}}$ \\
\hline$\Delta \mathrm{MMST} \bar{x}(\mathrm{SD})$ & $2,34(4,54)$ & $2,59(2,90)$ \\
\hline$\Delta \mathrm{MMST} \tilde{x}$ (IQR) & $0,95(4,84)$ & $2,90(4,83)$ \\
\hline p-Wert ${ }^{+}$ & $0,056^{\mathrm{c}}$ & $0,595^{\mathrm{c}}$ \\
\hline
\end{tabular}

\begin{tabular}{|c|c|c|}
\hline \multirow[t]{2}{*}{$A P O E$} & \multicolumn{2}{|c|}{ Männer } \\
\hline & ع4-Träger & \\
\hline Total $(\mathrm{n}=15)$ & $8(53,3 \%)$ & \\
\hline Slow $(\mathrm{n}=11)$ & $6(54,5 \%)$ & \\
\hline Rapid (n= 4) & $2(50,0 \%)$ & \\
\hline p-Wert* & $1,000^{\mathrm{a}}$ & \\
\hline$\Delta \mathrm{MMST} \bar{x}(\mathrm{SD})$ & $5,59(3,95)$ & \\
\hline$\Delta \mathrm{MMST} \tilde{x}(\mathrm{IQR})$ & $4,19(3,33)$ & \\
\hline p-Wert ${ }^{+}$ & $0,463^{\mathrm{c}}$ & \\
\hline$C L U$ & C-Träger & T-Träger \\
\hline Total $(\mathrm{n}=15)$ & $12(80,0 \%)$ & $10(66,7 \%)$ \\
\hline Slow $(n=11)$ & $9(81,8 \%)$ & $8(72,7 \%)$ \\
\hline Rapid $(n=4)$ & $3(75,0 \%)$ & $2(50,0 \%)$ \\
\hline p-Wert* & $1,000^{\mathrm{a}}$ & $0,560^{\mathrm{a}}$ \\
\hline$\Delta \mathrm{MMST} \bar{x}(\mathrm{SD})$ & $3,66(2,67)$ & $4,70(4,01)$ \\
\hline$\Delta \mathrm{MMST} \tilde{x}(\mathrm{IQR})$ & $3,93(2,86)$ & $4,19(2,41)$ \\
\hline p-Wert ${ }^{+}$ & $0,359^{\mathrm{b}}$ & $1,000^{\mathrm{c}}$ \\
\hline PICALM & C-Träger & T-Träger \\
\hline Total $(\mathrm{n}=15)$ & $9(60,0 \%)$ & $15(100,0 \%)$ \\
\hline Slow $(n=11)$ & $6(54,5 \%)$ & $11(100,0 \%)$ \\
\hline Rapid (n=4) & $3(75,0 \%)$ & $4(100,0 \%)$ \\
\hline p-Wert* & $0,604^{\mathrm{a}}$ & - \\
\hline$\Delta \mathrm{MMST} \bar{x}(\mathrm{SD})$ & $5,38(4,30)$ & $4,49(3,72)$ \\
\hline$\Delta \mathrm{MMST} \tilde{x}(\mathrm{IQR})$ & $4,41(3,93)$ & $3,97(3,03)$ \\
\hline p-Wert ${ }^{+}$ & $0,271^{\mathrm{b}}$ & - \\
\hline BIN1 & A-Träger & G-Träger \\
\hline Total $(\mathrm{n}=15)$ & $15(100,0 \%)$ & $6(40,0 \%)$ \\
\hline Slow $(n=11)$ & $11(100,0 \%)$ & $6(54,5)$ \\
\hline Rapid $(n=4)$ & $4(100,0 \%)$ & $0(0,0 \%)$ \\
\hline p-Wert* & - & $0,103^{\mathrm{a}}$ \\
\hline$\Delta \mathrm{MMST} \bar{x}(\mathrm{SD})$ & $4,49(3,72)$ & $3,30(2,12)$ \\
\hline$\Delta \mathrm{MMST} \tilde{x}(\mathrm{IQR})$ & $3,97(3,03)$ & $3,93(2,00)$ \\
\hline $\mathrm{p}-$ Wert ${ }^{+}$ & - & $0,529^{c}$ \\
\hline
\end{tabular}


Tabelle 17 (Fortsetzung)

\begin{tabular}{|c|c|c|}
\hline \multirow[b]{2}{*}{ IL-8 } & \multicolumn{2}{|c|}{ Frauen } \\
\hline & A-Träger & T-Träger \\
\hline Total $(n=25)$ & $15(60,0 \%)$ & $23(92,0 \%)$ \\
\hline Slow $(n=19)$ & $12(63,2 \%)$ & $17(89,5 \%)$ \\
\hline Rapid $(n=6)$ & $3(50,0 \%)$ & $6(100,0 \%)$ \\
\hline p-Wert* & $0,653^{\mathrm{a}}$ & $1,000^{\mathrm{a}}$ \\
\hline$\Delta \mathrm{MMST} \bar{x}(\mathrm{SD})$ & $2,90(5,00)$ & $3,04(4,50)$ \\
\hline$\Delta \mathrm{MMST} \tilde{x}(\mathrm{IQR})$ & $1,77(4,77)$ & $2,90(6,50)$ \\
\hline $\mathrm{p}-\mathrm{Wert}^{+}$ & $0,614^{\mathrm{c}}$ & $0,593^{\mathrm{c}}$ \\
\hline GWA14q32.13 & A-Träger & T-Träger \\
\hline Total $(n=25)$ & $17(68,0 \%)$ & $18(72,0 \%)$ \\
\hline Slow $(n=19)$ & $12(63,2 \%)$ & $14(73,7 \%)$ \\
\hline Rapid $(n=6)$ & $5(83,3 \%)$ & $4(66,7 \%)$ \\
\hline p-Wert* & $0,624^{\mathrm{a}}$ & $1,000^{\mathrm{a}}$ \\
\hline$\Delta \mathrm{MMST} \bar{x}(\mathrm{SD})$ & $3,54(4,72)$ & $2,48(3,93)$ \\
\hline$\Delta \mathrm{MMST} \tilde{x}(\mathrm{IQR})$ & $3,71(7,04)$ & $1,62(5,91)$ \\
\hline p-Wert ${ }^{+}$ & $0,367^{\mathrm{c}}$ & $0,781^{\mathrm{c}}$ \\
\hline$A C E$ & C-Träger & T-Träger \\
\hline Total $(n=25)$ & $17(68,0 \%)$ & $21(84,0 \%)$ \\
\hline Slow $(n=19)$ & $12(63,2 \%)$ & $16(84,2 \%)$ \\
\hline Rapid $(n=6)$ & $5(83,3 \%)$ & $5(83,3 \%)$ \\
\hline p-Wert* & $0,624^{\mathrm{a}}$ & $1,000^{\mathrm{a}}$ \\
\hline$\Delta \mathrm{MMST} \bar{x}(\mathrm{SD})$ & $3,79(4,56)$ & $2,94(4,60)$ \\
\hline$\Delta \mathrm{MMST} \tilde{x}(\mathrm{IQR})$ & $2,90(6,10)$ & $2,29(6,16)$ \\
\hline p-Wert ${ }^{+}$ & $0,083^{\mathrm{c}}$ & $0,867^{\mathrm{c}}$ \\
\hline TNK1 & A-Träger & T-Träger \\
\hline Total $(n=25)$ & $20(80,0 \%)$ & $22(88,0 \%)$ \\
\hline Slow $(n=19)$ & $14(73,7 \%)$ & $16(84,2 \%)$ \\
\hline Rapid $(n=6)$ & $6(100,0 \%)$ & $6(100,0 \%)$ \\
\hline p-Wert* & $0,289^{\mathrm{a}}$ & $0,554^{\mathrm{a}}$ \\
\hline$\Delta \mathrm{MMST} \bar{x}(\mathrm{SD})$ & $3,21(4,64)$ & $3,29(4,49)$ \\
\hline$\Delta \mathrm{MMST} \tilde{x}(\mathrm{IQR})$ & $2,34(7,28)$ & $3,31(6,68)$ \\
\hline $\mathrm{p}$-Wert ${ }^{+}$ & $0,440^{\mathrm{c}}$ & $0,126^{\mathrm{c}}$ \\
\hline LDLR & A-Träger & G-Träger \\
\hline Total $(n=25)$ & $16(64,0 \%)$ & $23(92,0 \%)$ \\
\hline Slow $(\mathrm{n}=19)$ & $12(63,2 \%)$ & $17(89,5 \%)$ \\
\hline Rapid $(n=6)$ & $4(66,7 \%)$ & $6(100,0 \%)$ \\
\hline p-Wert* & $1,000^{\mathrm{a}}$ & $1,000^{\mathrm{a}}$ \\
\hline$\Delta \mathrm{MMST} \bar{x}(\mathrm{SD})$ & $2,90(4,40)$ & $3,23(4,37)$ \\
\hline$\Delta \mathrm{MMST} \tilde{x}(\mathrm{IQR})$ & $2,60(6,95)$ & $2,90(6,38)$ \\
\hline $\mathrm{p}$-Wert ${ }^{+}$ & $0,942^{\mathrm{b}}$ & $0,040^{\mathrm{c}}$ \\
\hline
\end{tabular}

\begin{tabular}{|c|c|c|}
\hline \multirow[b]{2}{*}{$I L-8$} & \multicolumn{2}{|c|}{ Männer } \\
\hline & A-Träger & T-Träger \\
\hline Total $(\mathrm{n}=15)$ & $10(66,7 \%)$ & $13(86,7 \%)$ \\
\hline Slow $(\mathrm{n}=11)$ & $7(63,6 \%)$ & $10(90,9 \%)$ \\
\hline Rapid $(n=4)$ & $3(75,0 \%)$ & $3(75,0 \%)$ \\
\hline p-Wert* & $1,000^{\mathrm{a}}$ & $0,476^{\mathrm{a}}$ \\
\hline$\Delta \mathrm{MMST} \bar{x}(\mathrm{SD})$ & $5,09(4,08)$ & $3,74(2,58)$ \\
\hline$\Delta \mathrm{MMST} \tilde{x}(\mathrm{IQR})$ & $4,62(3,49)$ & $3,90(2,65)$ \\
\hline p-Wert ${ }^{+}$ & $0,401^{\mathrm{b}}$ & $0,485^{\mathrm{b}}$ \\
\hline GWA14q32.13 & A-Träger & T-Träger \\
\hline Total $(\mathrm{n}=15)$ & $9(60,0 \%)$ & $14(93,3 \%)$ \\
\hline Slow $(n=11)$ & $6(54,5 \%)$ & $11(100,0 \%)$ \\
\hline Rapid $(\mathrm{n}=4)$ & $3(75,0 \%)$ & $3(75,0 \%)$ \\
\hline p-Wert* & $0,604^{\mathrm{a}}$ & $0,267^{\mathrm{a}}$ \\
\hline$\Delta \mathrm{MMST} \bar{x}(\mathrm{SD})$ & $4,25(4,82)$ & $3,75(2,48)$ \\
\hline$\Delta \operatorname{MMST} \tilde{x}$ & $2,93(5,81)$ & $3,93(2,41)$ \\
\hline p-Wert ${ }^{+}$ & $0,272^{\mathrm{c}}$ & $0,001^{\mathrm{b}}$ \\
\hline$A C E$ & C-Träger & T-Träger \\
\hline Total $(\mathrm{n}=15)$ & $12(80,0 \%)$ & $12(80,0 \%)$ \\
\hline Slow $(n=11)$ & $9(81,8 \%)$ & $8(72,7 \%)$ \\
\hline Rapid (n=4) & $3(75,0 \%)$ & $4(100,0 \%)$ \\
\hline p-Wert* & $1,000^{\mathrm{a}}$ & $0,516^{\mathrm{a}}$ \\
\hline$\Delta \mathrm{MMST} \bar{x}(\mathrm{SD})$ & $4,47(4,09)$ & $5,02(3,82)$ \\
\hline$\Delta \mathrm{MMST} \tilde{x}(\mathrm{IQR})$ & $4,19(2,85)$ & $4,40(3,89)$ \\
\hline p-Wert ${ }^{+}$ & $0,969^{b}$ & $0,287^{\mathrm{b}}$ \\
\hline TNK1 & A-Träger & T-Träger \\
\hline Total $(n=15)$ & $12(80,0 \%)$ & $13(86,7 \%)$ \\
\hline Slow $(n=11)$ & $8(72,7 \%)$ & $10(90,9 \%)$ \\
\hline Rapid (n=4) & $4(100,0 \%)$ & $3(75,0 \%)$ \\
\hline p-Wert* & $0,516^{\mathrm{a}}$ & $0,476^{\mathrm{a}}$ \\
\hline$\Delta \mathrm{MMST} \bar{x}(\mathrm{SD})$ & $5,03(3,79)$ & $4,68(3,58)$ \\
\hline$\Delta$ MMST $\tilde{x}(\mathrm{IQR})$ & $4,19(3,71)$ & $3,97(2,53)$ \\
\hline p-Wert ${ }^{+}$ & $0,273^{\mathrm{b}}$ & $0,933^{\mathrm{c}}$ \\
\hline LDLR & A-Träger & G-Träger \\
\hline Total $(\mathrm{n}=15)$ & $8(53,3 \%)$ & $11(73,3 \%)$ \\
\hline Slow $(\mathrm{n}=11)$ & $7(63,6 \%)$ & $8(72,7 \%)$ \\
\hline Rapid $(n=4)$ & $1(25,0 \%)$ & $3(75,0 \%)$ \\
\hline p-Wert* & $0,282^{\mathrm{a}}$ & $1,000^{\mathrm{a}}$ \\
\hline$\Delta \mathrm{MMST} \bar{x}(\mathrm{SD})$ & $4,18(4,91)$ & $4,15(2,31)$ \\
\hline$\Delta \mathrm{MMST} \tilde{x}(\mathrm{IQR})$ & $3,93(4,91)$ & $3,97(3,03)$ \\
\hline $\mathrm{p}-$ Wert ${ }^{+}$ & $0,613^{c}$ & $0,735^{\mathrm{b}}$ \\
\hline
\end{tabular}


Tabelle 17 (Fortsetzung)

\begin{tabular}{|c|c|c|}
\hline \multirow[b]{2}{*}{ EXOC3L2 } & \multicolumn{2}{|c|}{ Frauen } \\
\hline & C-Träger & T-Träger \\
\hline Total $(n=25)$ & $8(32,0 \%)$ & $25(100,0 \%)$ \\
\hline Slow $(n=19)$ & $6(31,6 \%)$ & $19(100,0 \%)$ \\
\hline Rapid $(n=6)$ & $2(33,3 \%)$ & $6(100,0 \%)$ \\
\hline p-Wert* & $1,000^{\mathrm{a}}$ & - \\
\hline$\Delta \mathrm{MMST} \bar{x}(\mathrm{SD})$ & $4,85(4,70)$ & $2,86(4,38)$ \\
\hline$\Delta \operatorname{MMST} \tilde{x}(\mathrm{IQR})$ & $3,85(4,40)$ & $2,29(6,11)$ \\
\hline $\mathrm{p}$-Wert ${ }^{+}$ & $0,107^{\mathrm{c}}$ & - \\
\hline CST3 & C-Träger & T-Träger \\
\hline Total $(n=25)$ & $22(88,0 \%)$ & $12(48,0 \%)$ \\
\hline Slow $(n=19)$ & $16(84,2 \%)$ & $10(52,6 \%)$ \\
\hline Rapid $(n=6)$ & $6(100,0 \%)$ & $2(33,3 \%)$ \\
\hline p-Wert* & $0,554^{\mathrm{a}}$ & $0,645^{\mathrm{a}}$ \\
\hline$\Delta \mathrm{MMST} \bar{x}(\mathrm{SD})$ & $2,86(4,62)$ & $3,43(4,39)$ \\
\hline$\Delta \mathrm{MMST} \tilde{x}(\mathrm{IQR})$ & $2,03(6,79)$ & $3,72(5,25)$ \\
\hline $\mathrm{p}$-Wert ${ }^{+}$ & $0,670^{\mathrm{c}}$ & $0,355^{\mathrm{c}}$ \\
\hline
\end{tabular}

\begin{tabular}{|c|c|c|}
\hline \multirow[b]{2}{*}{ EXOC3L2 } & \multicolumn{2}{|c|}{ Männer } \\
\hline & C-Träger & T-Träger \\
\hline Total $(n=15)$ & $3(20,0 \%)$ & $15(100,0 \%)$ \\
\hline Slow $(n=11)$ & $2(18,2 \%)$ & $11(100,0 \%)$ \\
\hline Rapid $(n=4)$ & $1(25,0 \%)$ & $4(100,0 \%)$ \\
\hline p-Wert* & $1,000^{\mathrm{a}}$ & - \\
\hline$\Delta \mathrm{MMST} \bar{x}(\mathrm{SD})$ & $4,96(1,78)$ & $4,49(3,72)$ \\
\hline$\Delta \mathrm{MMST} \tilde{x}(\mathrm{IQR})$ & 3,97 & $3,97(3,03)$ \\
\hline p-Wert ${ }^{+}$ & $0,633^{\mathrm{c}}$ & - \\
\hline CST3 & C-Träger & T-Träger \\
\hline Total $(n=15)$ & $15(100,0 \%)$ & $4(26,7 \%)$ \\
\hline Slow $(n=11)$ & $11(100,0 \%)$ & $3(27,3 \%)$ \\
\hline Rapid (n=4) & $4(100,0 \%)$ & $1(25,0 \%)$ \\
\hline $\mathrm{p}$-Wert* & - & $1,000^{\mathrm{a}}$ \\
\hline$\Delta \mathrm{MMST} \bar{x}(\mathrm{SD})$ & $4,49(3,72)$ & $3,42(3,39)$ \\
\hline$\Delta \mathrm{MMST} \tilde{x}(\mathrm{IQR})$ & $3,97(3,03)$ & $3,81(6,49)$ \\
\hline & - & $0,753^{\mathrm{c}}$ \\
\hline
\end{tabular}

AMMST: Punktverlust im MMST/Jahr (MMST-Progression)

Slow: $\triangle \mathrm{MMST} \leq 5$

Rapid: $\triangle \mathrm{MMST}>5$

* slow/rapid vs. Allelträger/Nicht-Allelträger

${ }^{+}$MMST-Progression vs. Allelträger/Nicht-Allelträger

${ }^{\mathrm{a}}$ Exakter Test nach Fisher

${ }^{\mathrm{b}}$ T-Test

${ }^{\mathrm{c}}$ Mann-Whitney-Test

${ }^{\mathrm{d}}$ Einfaktorielle ANOVA

${ }^{\mathrm{e}}$ Kruskal-Wallis-Test

\subsubsection{BIN1}

$\underline{\text { Frauen }}$

Die als langsam (,,slow") und als schnell verlaufend (,,rapid“) definierten Fälle unterschieden sich weder in der Verteilung von A-Trägern und Nicht-A-Trägern noch von G-Trägern und Nicht-G-Trägern signifikant (siehe Tabelle 17). A-Träger wiesen jedoch mit einem Median von 1,0 Punkten (IQR 4,8) eine deutlich niedrigere MMST-Progression als die Nicht-ATräger auf ( $\tilde{x}=5,8$ Punkte, IQR 4,2). Dieser Unterschied war jedoch nicht statistisch signifikant ( $p=0,056$, siehe Abb. 15). G-Träger und Nicht-G-Träger unterschieden sich nicht deutlich in der MMST-Progression (siehe Tabelle 17). 


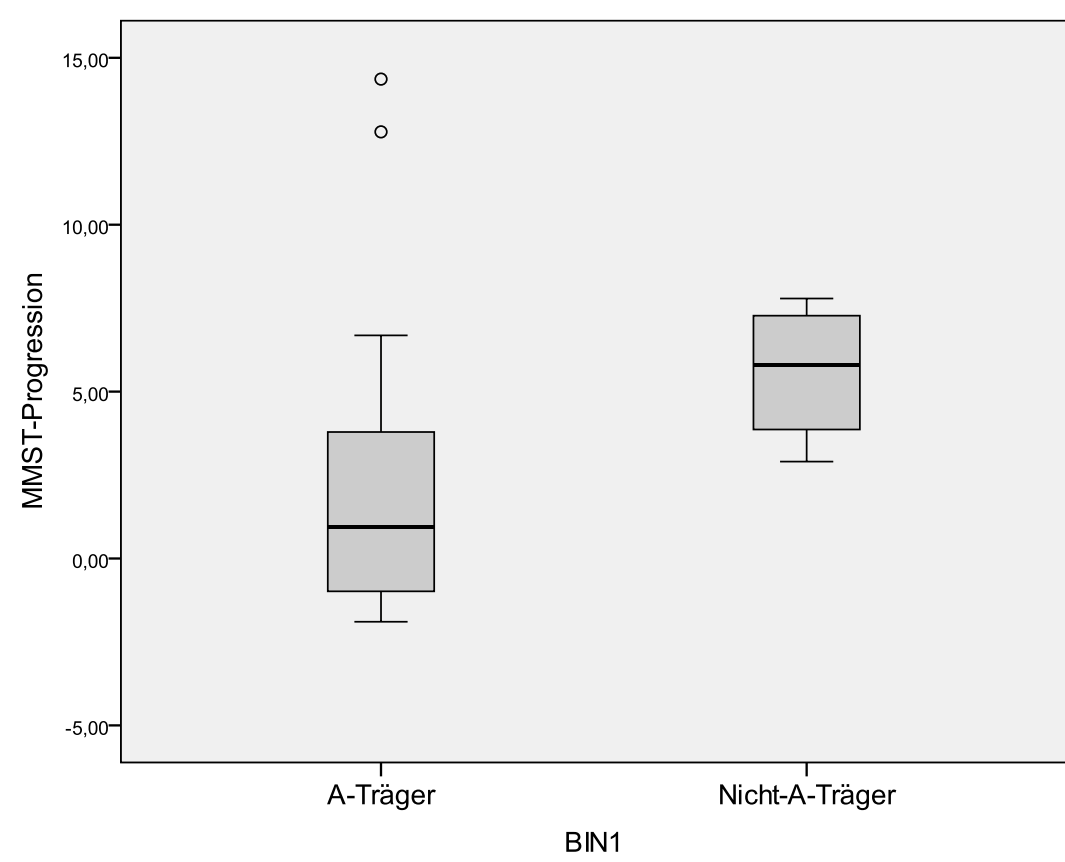

Mann-Whitney-Test: $p=0,056$

Abb. 15: BIN1: MMST-Progression von A-Trägern und Nicht-A-Trägern bei Frauen

\section{$\underline{\text { Männer }}$}

Alle männlichen Studienteilnehmer hatten mindestens ein A-Allel, sodass ein Vergleich mit Nicht-A-Allel-Trägern nicht durchgeführt werden konnte. Zwischen G-Trägern und Nicht-GTrägern konnte weder ein signifikanter Unterschied in der Verteilung auf die langsam und schnell verlaufenden Gruppen noch in der MMST-Progression festgestellt werden (siehe Tabelle 17).

\subsubsection{GWA14q32.13}

\section{$\underline{\text { Frauen }}$}

Die als langsam und als schnell verlaufend definierten Fälle unterschieden sich weder in der Verteilung von A-Trägern und Nicht-A-Trägern noch von T-Trägern und Nicht-T-Trägern signifikant. Auch hinsichtlich der MMST-Progression konnte zwischen A-Trägern und NichtA-Trägern bzw. zwischen T-Trägern und Nicht-T-Trägern kein signifikanter Unterschied festgestellt werden (siehe Tabelle 17). 
$\underline{\text { Männer }}$

Zwischen A-Trägern und Nicht-A-Trägern ergab sich weder in der Anzahl der langsam und schnell verlaufenden Fälle noch in der MMST-Progression ein signifikanter Unterschied. Auch T-und Nicht-T-Träger unterschieden sich nicht in der Anzahl von langsam und schnell verlaufenden Fällen (siehe Tabelle 17). T-Träger wiesen jedoch mit einem Mittelwert von 3,8 Punkten (SD 2,5) eine signifikant niedrigere MMST-Progression als der einzige Nicht-TTräger auf ( $\bar{x}=14,8$ Punkte, siehe Abb. 16).

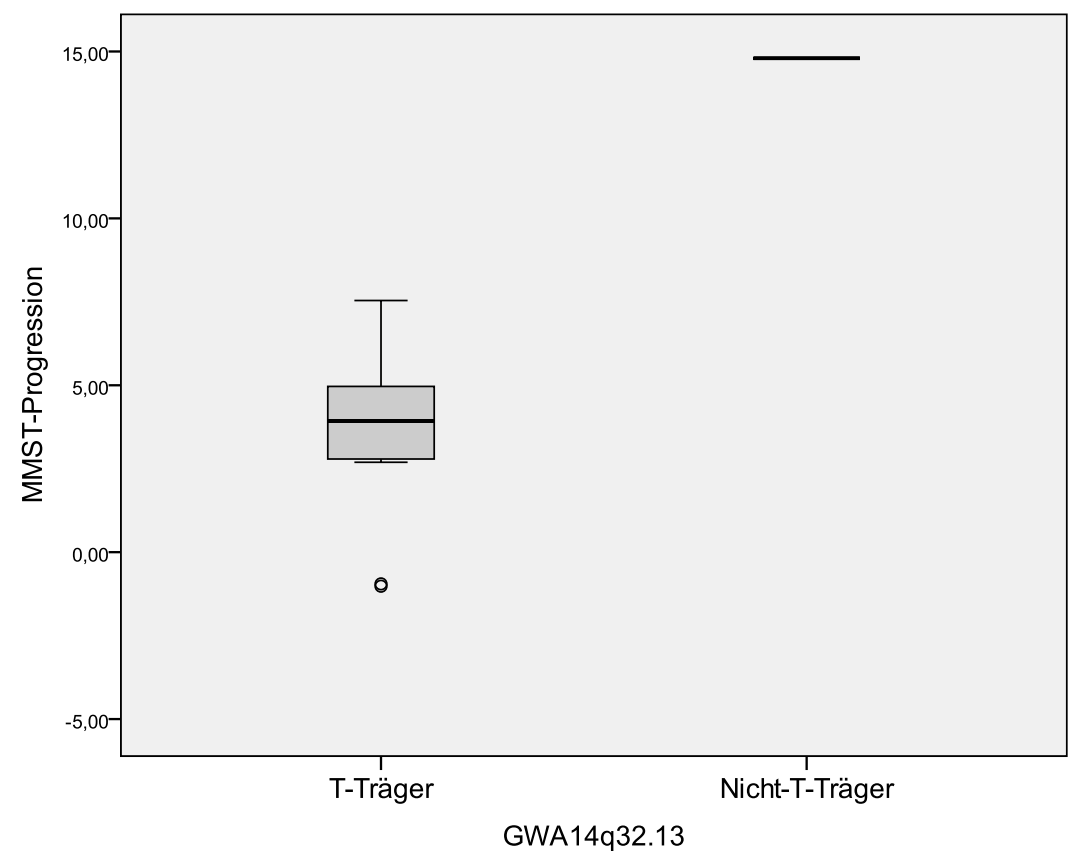

T-Test: $p=0,001$

Abb. 16: GWA14q32.12: MMST-Progression von T-Trägern und Nicht-T-Trägern bei Männern

\subsubsection{ACE}

Frauen

Hinsichtlich der Anzahl langsam und schnell verlaufender Fälle konnte zwischen C-Trägern und Nicht-C-Trägern kein deutlicher Unterschied gefunden werden (siehe Tabelle 17). CTräger zeigten mit einem Median von 2,9 Punkten (IQR 6,1) allerdings eine schnellere Progression als Nicht-C-Träger ( $\tilde{x}=-0,9$ Punkte, IQR 5,7). Dieses Ergebnis war jedoch nicht statistisch signifikant ( $p=0,083$, Mann-Whitney-Test, Abb. 17). T- und Nicht-T-Träger 
unterschieden sich weder in der Anzahl der schnell und langsam verlaufenden Fälle noch in der MMST-Progression deutlich voneinander (siehe Tabelle 17).

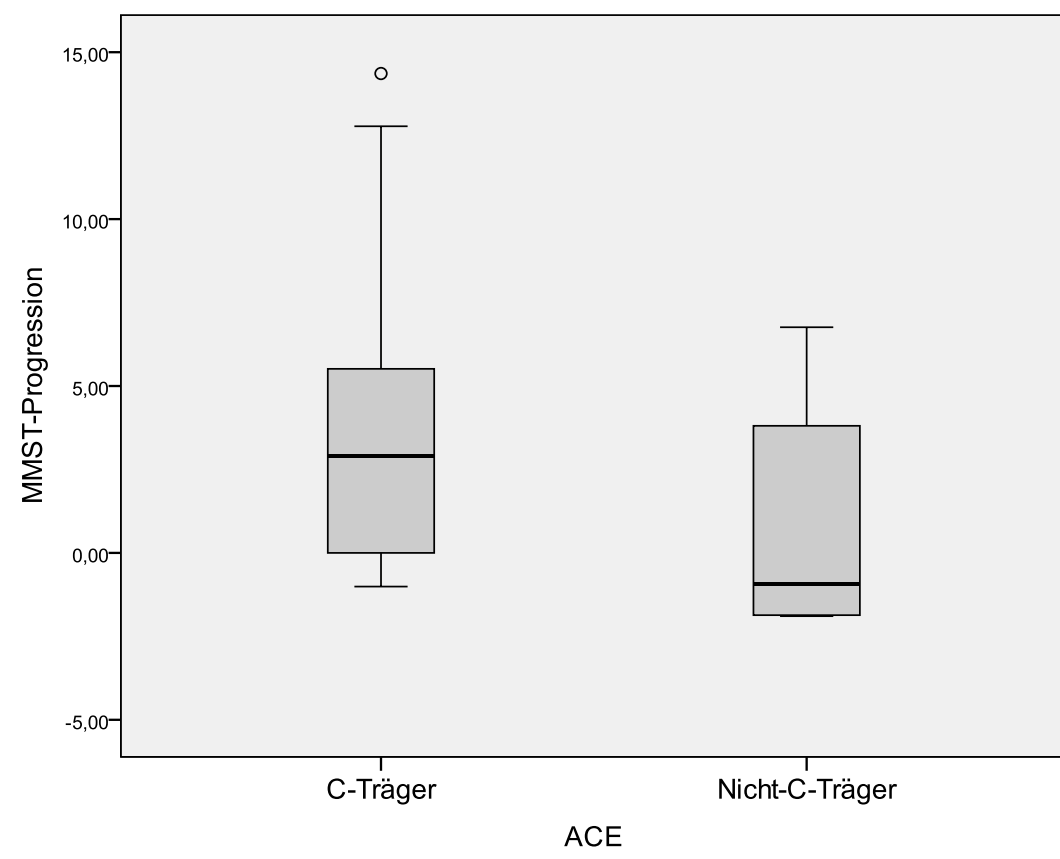

Mann-Whitney-Test: $p=0,083$

Abb. 17: ACE: MMST-Progression von C-Trägern und Nicht-C-Trägern bei Frauen

\section{$\underline{\text { Männer }}$}

Für C- und Nicht-C-Träger bzw. T- und Nicht-T-Träger konnte weder ein signifikanter Unterschied in der Anzahl der schnell und langsam verlaufenden Fälle noch in der MMSTProgression festgestellt werden (siehe Tabelle 17).

\subsubsection{LDLR}

\section{$\underline{\text { Frauen }}$}

A-Träger und Nicht-A-Träger bzw. G-Träger und Nicht-G-Träger unterschieden sich nicht signifikant hinsichtlich der Verteilung auf die als langsam und schnell definierten Gruppen. Für A-Träger und Nicht-A-Träger ließ sich zudem kein statistisch signifikanter Unterschied in der MMST-Progression feststellen (siehe Tabelle 17). G-Träger wiesen jedoch mit einem Median von 2,9 Punkten (IQR 6,4) eine höhere MMST-Progression als Nicht-G-Träger auf 
$(\tilde{x}=-1,5$ Punkte, $\mathrm{n}=2$ ). Dieser Unterschied war statistisch signifikant ( $p=0,040$, MannWhitney-Test, siehe Abb. 18).

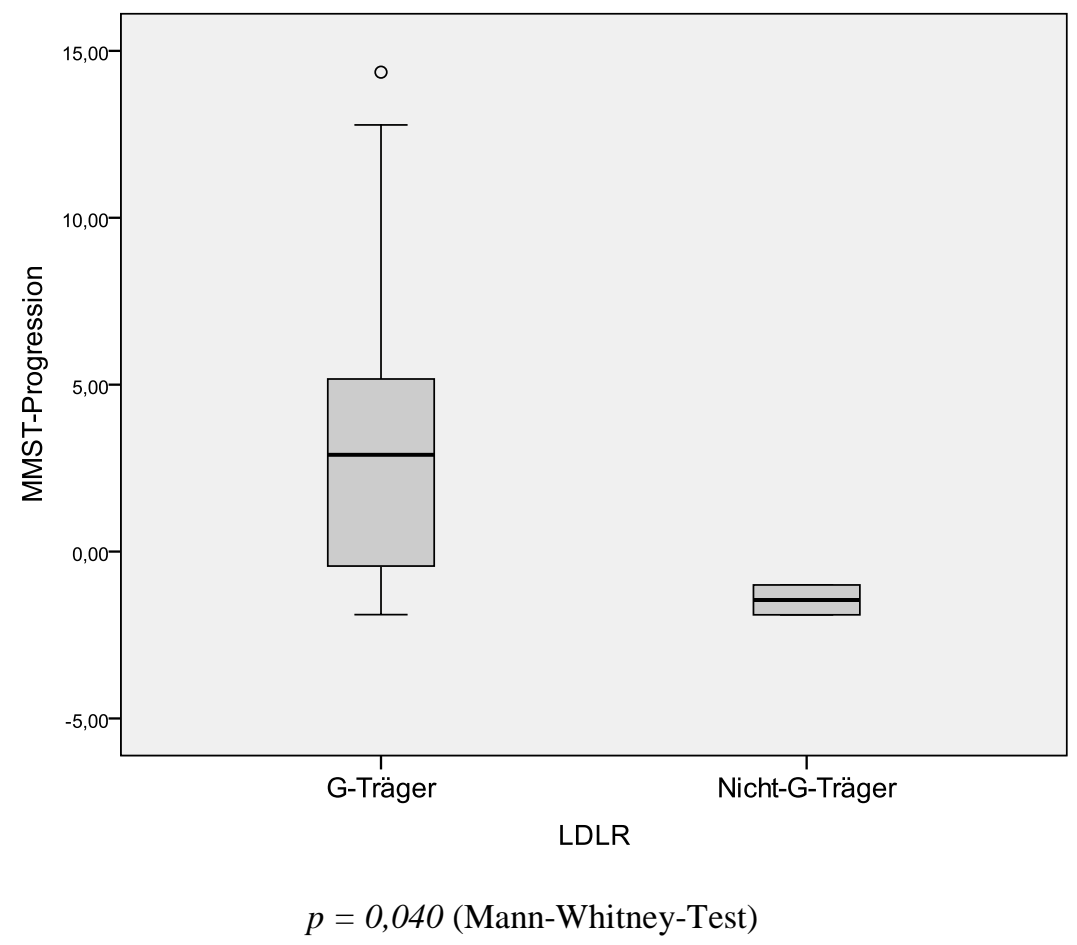

Abb. 18: LDLR: MMST-Progression von G-Trägern und Nicht G-Trägern bei Frauen

\section{Männer}

Hier zeigte sich zwischen A-Trägern und Nicht-A-Trägern sowie zwischen G-Trägern und Nicht-G-Trägern weder hinsichtlich der Anzahl langsam oder schnell verlaufender Fälle noch hinsichtlich der MMST-Progression ein statistisch signifikanter Unterschied (siehe Tabelle 17).

\subsubsection{Allelverteilung bei Patienten mit EOAD und LOAD}

Tabelle 18 zeigt für Patienten mit frühem Krankheitsbeginn $(\leq 65$ Jahre $)$ und für Studienteilnehmer mit spätem Krankheitsbeginn (> 65 Jahre) eine Übersicht der AllelträgerVerteilung sowie der jeweiligen Krankheitsprogression. Signifikante bzw. Trend-Ergebnisse werden im Anschluss ausführlich beschrieben. 
Tabelle 18: Untersuchte Polymorphismen nach Krankheitsbeginn stratifiziert: Allelverteilung und jeweilige Progression

\begin{tabular}{|c|c|c|}
\hline \multirow[b]{2}{*}{ Apo $E$} & \multicolumn{2}{|c|}{ EOAD } \\
\hline & $\varepsilon 4$-Träger & \\
\hline Total $(n=17)$ & $8(47,1 \%)$ & \\
\hline Slow $(n=13)$ & $7(53,8 \%)$ & \\
\hline Rapid $(n=4)$ & $1(25,0 \%)$ & \\
\hline p-Wert* & $0,576^{\mathrm{a}}$ & \\
\hline$\Delta \mathrm{MMST} \bar{x}(\mathrm{SD})$ & $3,57(2,20)$ & \\
\hline$\Delta \mathrm{MMST} \tilde{x}(\mathrm{IQR})$ & $3,84(2,07)$ & \\
\hline $\mathrm{p}-\mathrm{Wert}{ }^{+}$ & $0,479^{\mathrm{b}}$ & \\
\hline$C L U$ & C-Träger & T-Träger \\
\hline Total $(n=17)$ & $15(88,2 \%)$ & $11(64,7 \%)$ \\
\hline Slow $(n=13)$ & $11(84,6 \%)$ & $10(76,9 \%)$ \\
\hline Rapid $(n=4)$ & $4(100,0 \%)$ & $1(25,0 \%)$ \\
\hline p-Wert* & $1,000^{\mathrm{a}}$ & $0,099^{\mathrm{a}}$ \\
\hline$\Delta \mathrm{MMST} \bar{x}(\mathrm{SD})$ & $3,09(2,96)$ & $2,32(2,57)$ \\
\hline$\Delta \mathrm{MMST} \tilde{x}(\mathrm{IQR})$ & $2,93(6,69)$ & $2,79(5,28)$ \\
\hline p-Wert ${ }^{+}$ & $0,791^{\mathrm{b}}$ & $0,189^{b}$ \\
\hline PICALM & C-Träger & T-Träger \\
\hline Total $(n=17)$ & $8(47,1 \%)$ & $17(100,0 \%)$ \\
\hline Slow $(n=13)$ & $6(46,2 \%)$ & $13(100,0 \%)$ \\
\hline Rapid $(n=4)$ & $2(50,0 \%)$ & $4(100,0 \%)$ \\
\hline $\mathrm{p}$-Wert* & $1,000^{\mathrm{a}}$ & - \\
\hline$\Delta \mathrm{MMST} \bar{x}(\mathrm{SD})$ & $3,57(3,14)$ & $3,02(2,91)$ \\
\hline$\Delta \mathrm{MMST} \tilde{x}(\mathrm{IQR})$ & $4,19(6,21)$ & $2,93(5,83)$ \\
\hline $\mathrm{p}-\mathrm{Wert}^{+}$ & $0,483^{\mathrm{b}}$ & - \\
\hline BIN1 & A-Träger & G-Träger \\
\hline Total $(n=17)$ & $15(88,2 \%)$ & $8(47,1 \%)$ \\
\hline Slow $(n=13)$ & $12(92,3 \%)$ & $7(53,8 \%)$ \\
\hline Rapid $(n=4)$ & $3(75,0 \%)$ & $1(25,0 \%)$ \\
\hline p-Wert* & $0,426^{\mathrm{a}}$ & $0,576^{\mathrm{a}}$ \\
\hline$\Delta \operatorname{MMST} \bar{x}(\mathrm{SD})$ & $2,78(2,93)$ & $2,48(2,83)$ \\
\hline$\Delta \mathrm{MMST} \tilde{x}(\mathrm{IQR})$ & $2,93(5,83)$ & $3,31(5,01)$ \\
\hline $\mathrm{p}-\mathrm{Wert}{ }^{+}$ & $0,365^{\mathrm{b}}$ & $0,484^{b}$ \\
\hline$I L-8$ & A-Träger & T-Träger \\
\hline Total $(n=17)$ & $11(64,7 \%)$ & $16(94,1 \%)$ \\
\hline Slow $(n=13)$ & $8(61,5 \%$ & $12(92,3 \%)$ \\
\hline Rapid $(n=4)$ & $3(75,0 \%)$ & $4(100,0 \%)$ \\
\hline p-Wert* & $1,000^{\mathrm{a}}$ & $1,000^{\mathrm{a}}$ \\
\hline$\Delta \mathrm{MMST} \bar{x}(\mathrm{SD})$ & $3,79(2,60)$ & $2,96(2,99)$ \\
\hline$\Delta \mathrm{MMST} \tilde{x}(\mathrm{IQR})$ & $3,97(3,13)$ & $2,92(6,26)$ \\
\hline $\mathrm{p}$-Wert ${ }^{+}$ & $0,148^{\mathrm{b}}$ & $0,749^{\mathrm{b}}$ \\
\hline
\end{tabular}

\begin{tabular}{|c|c|c|}
\hline \multirow[b]{2}{*}{ Apo $E$} & \multicolumn{2}{|c|}{ LOAD } \\
\hline & $\varepsilon 4$-Träger & \\
\hline Total $(\mathrm{n}=24)$ & $16(66,7 \%)$ & \\
\hline Slow $(\mathrm{n}=18)$ & $12(66,7 \%)$ & \\
\hline Rapid $(\mathrm{n}=6)$ & $4(66,7 \%)$ & \\
\hline p-Wert* & $1,000^{\mathrm{a}}$ & \\
\hline$\Delta \mathrm{MMST} \bar{x}(\mathrm{SD})$ & $3,64(5,04)$ & \\
\hline $\begin{array}{l}\Delta \text { MMST } \\
\text { p-Wert }\end{array}$ & $\begin{array}{c}3,76(7,45) \\
0,941^{\mathrm{b}}\end{array}$ & \\
\hline$C L U$ & C-Träger & T-Träger \\
\hline Total $(n=23)$ & $19(82,6 \%)$ & $12(52,2 \%)$ \\
\hline Slow $(n=17)$ & $15(88,2 \%)$ & $8(47,1 \%)$ \\
\hline Rapid $(n=6)$ & $4(66,7 \%)$ & $4(66,7 \%)$ \\
\hline p-Wert* & $0,270^{\mathrm{a}}$ & $0,640^{\mathrm{a}}$ \\
\hline$\Delta \mathrm{MMST} \bar{x}(\mathrm{SD})$ & $3,44(4,59)$ & $5,10(5,93)$ \\
\hline$\Delta \mathrm{MMST} \tilde{x}(\mathrm{IQR})$ & $3,73(5,72)$ & $3,85(11,13)$ \\
\hline $\mathrm{p}-\mathrm{Wert}^{+}$ & $0,655^{\mathrm{c}}$ & $0,194^{b}$ \\
\hline PICALM & C-Träger & T-Träger \\
\hline Total $(n=23)$ & $11(47,8 \%)$ & $20(87,0 \%)$ \\
\hline Slow $(n=17)$ & $7(41,2 \%)$ & $15(88,2 \%)$ \\
\hline Rapid $(\mathrm{n}=6)$ & $4(66,7 \%)$ & $5(83,3 \%)$ \\
\hline p-Wert* & $0,371^{\mathrm{a}}$ & $1,000^{\mathrm{a}}$ \\
\hline$\Delta \mathrm{MMST} \bar{x}(\mathrm{SD})$ & $5,85(5,77)$ & $3,56(4,55)$ \\
\hline$\Delta \mathrm{MMST} \tilde{x}(\mathrm{IQR})$ & $3,90(11,83)$ & $3,73(6,01)$ \\
\hline p-Wert ${ }^{+}$ & $0,055^{\mathrm{b}}$ & $0,556^{\mathrm{b}}$ \\
\hline BIN1 & A-Träger & G-Träger \\
\hline Total $(\mathrm{n}=23)$ & $21(91,3 \%)$ & $13(56,5 \%)$ \\
\hline Slow $(n=17)$ & $16(94,1 \%)$ & $11(64,7 \%)$ \\
\hline Rapid $(n=6)$ & $5(83,3 \%)$ & $2(33,3 \%)$ \\
\hline $\mathrm{p}$-Wert* & $0,462^{\mathrm{a}}$ & $0,341^{\mathrm{a}}$ \\
\hline$\Delta \mathrm{MMST} \bar{x}(\mathrm{SD})$ & $3,56(5,10)$ & $3,00(2,67)$ \\
\hline$\Delta \mathrm{MMST} \tilde{x}(\mathrm{IQR})$ & $3,66(6,11)$ & $3,79(4,35)$ \\
\hline $\mathrm{p}$-Wert ${ }^{+}$ & $0,206^{\mathrm{c}}$ & $0,964^{\mathrm{c}}$ \\
\hline$I L-8$ & A-Träger & T-Träger \\
\hline Total $(\mathrm{n}=23)$ & $14(60,9 \%)$ & $20(87,0 \%)$ \\
\hline Slow $(\mathrm{n}=17)$ & $11(64,7 \%)$ & $15(88,2 \%)$ \\
\hline Rapid $(n=6)$ & $3(50,0 \%)$ & $5(83,3 \%)$ \\
\hline p-Wert* & $0,643^{\mathrm{a}}$ & $1,000^{\mathrm{a}}$ \\
\hline$\Delta \mathrm{MMST} \bar{x}(\mathrm{SD})$ & $3,76(5,95)$ & $3,56(4,54)$ \\
\hline$\Delta \mathrm{MMST} \tilde{x}(\mathrm{IQR})$ & $2,03(7,80)$ & $3,76(6,08)$ \\
\hline $\mathrm{p}-\mathrm{Wert}{ }^{+}$ & $0,412^{c}$ & $0,561^{b}$ \\
\hline
\end{tabular}


Tabelle 18 (Fortsetzung)

\begin{tabular}{|c|c|c|}
\hline \multirow[b]{2}{*}{ GWA14q32.13 } & \multicolumn{2}{|c|}{ EOAD } \\
\hline & A-Träger & T-Träger \\
\hline Total $(n=17)$ & $11(64,7 \%)$ & $15(88,2 \%)$ \\
\hline Slow $(n=13)$ & $7(53,8 \%)$ & $12(92,3 \%)$ \\
\hline Rapid ( $n=4)$ & $4(100,0 \%)$ & $3(75,0 \%)$ \\
\hline p-Wert* & $0,237^{\mathrm{a}}$ & $0,426^{\mathrm{a}}$ \\
\hline$\Delta \mathrm{MMST} \bar{x}(\mathrm{SD})$ & $3,62(2,91)$ & $2,72(2,92)$ \\
\hline$\Delta \mathrm{MMST} \tilde{x}(\mathrm{IQR})$ & $2,93(3,99)$ & $2,90(5,83)$ \\
\hline p-Wert ${ }^{+}$ & $0,259^{\mathrm{b}}$ & $0,265^{\mathrm{b}}$ \\
\hline$A C E$ & C-Träger & T-Träger \\
\hline Total $(n=17)$ & $12(70,6 \%)$ & $14(82,4 \%)$ \\
\hline Slow $(n=13)$ & $9(69,2 \%)$ & $11(84,6 \%)$ \\
\hline Rapid (n=4) & $3(75,0 \%)$ & $3(75,0 \%)$ \\
\hline p-Wert* & $1,000^{\mathrm{a}}$ & $1,000^{\mathrm{a}}$ \\
\hline$\Delta \mathrm{MMST} \bar{x}(\mathrm{SD})$ & $3,33(2,84)$ & $2,94(2,83)$ \\
\hline$\Delta \mathrm{MMST} \tilde{x}(\mathrm{IQR})$ & $3,45(4,93)$ & $2,92(5,40)$ \\
\hline $\mathrm{p}-\mathrm{Wert}^{+}$ & $0,515^{\mathrm{b}}$ & $0,821^{\mathrm{b}}$ \\
\hline TNK1 & A-Träger & T-Träger \\
\hline Total $(n=17)$ & $14(82,4 \%)$ & $14(82,4 \%)$ \\
\hline Slow $(n=13)$ & $10(76,9 \%)$ & $11(84,6 \%)$ \\
\hline Rapid (n=4) & $4(100,0 \%)$ & $3(75,0 \%)$ \\
\hline p-Wert* & $0,541^{\mathrm{a}}$ & $1,000^{\mathrm{a}}$ \\
\hline$\Delta \mathrm{MMST} \bar{x}(\mathrm{SD})$ & $3,17(2,98)$ & $3,27(2,51)$ \\
\hline$\Delta \mathrm{MMST} \tilde{x}(\mathrm{IQR})$ & $3,31(6,25)$ & $3,32(3,16)$ \\
\hline p-Wert ${ }^{+}$ & $0,659^{\mathrm{b}}$ & $0,432^{\mathrm{c}}$ \\
\hline$L D L R$ & A-Träger & G-Träger \\
\hline Total $(n=17)$ & $10(58,8 \%)$ & $13(76,5 \%)$ \\
\hline Slow $(n=13)$ & $8(61,5 \%)$ & $9(69,2 \%)$ \\
\hline Rapid (n=4) & $2(50,0 \%)$ & $4(100,0 \%)$ \\
\hline p-Wert* & $1,000^{\mathrm{a}}$ & $0,519^{\mathrm{a}}$ \\
\hline$\Delta \mathrm{MMST} \bar{x}(\mathrm{SD})$ & $2,90(2,99)$ & $3,50(2,83)$ \\
\hline$\Delta \mathrm{MMST} \tilde{x}(\mathrm{IQR})$ & $3,32(6,36)$ & $3,71(4,91)$ \\
\hline p-Wert ${ }^{+}$ & $0,841^{\mathrm{b}}$ & $0,234^{\mathrm{b}}$ \\
\hline$E X O C 3 L 2$ & C-Träger & T-Träger \\
\hline Total $(n=17)$ & $1(5,9 \%)$ & $17(100,0 \%)$ \\
\hline Slow $(n=13)$ & $1(7,7 \%)$ & $13(100,0 \%)$ \\
\hline Rapid (n=4) & $0(0,0 \%)$ & $4(100,0 \%)$ \\
\hline p-Wert* & $1,000^{\mathrm{a}}$ & - \\
\hline$\Delta \mathrm{MMST} \bar{x}(\mathrm{SD})$ & 3,97 & $3,02(2,91)$ \\
\hline$\Delta \mathrm{MMST} \tilde{x}(\mathrm{IQR})$ & 3,97 & $2,93(5,83)$ \\
\hline $\mathrm{p}$-Wert ${ }^{+}$ & $0,749^{\mathrm{b}}$ & - \\
\hline
\end{tabular}

\begin{tabular}{|c|c|c|}
\hline \multirow[b]{2}{*}{$G W A 14 q 32.13$} & \multicolumn{2}{|c|}{ LOAD } \\
\hline & A-Träger & T-Träger \\
\hline Total $(n=23)$ & $15(65,2 \%)$ & $17(73,9 \%)$ \\
\hline Slow $(n=17)$ & $11(64,7 \%)$ & $13(76,5 \%)$ \\
\hline Rapid $(n=6)$ & $4(66,7 \%)$ & $4(66,7 \%)$ \\
\hline p-Wert* & $1,000^{\mathrm{a}}$ & $0,632^{\mathrm{a}}$ \\
\hline$\Delta \mathrm{MMST} \bar{x}(\mathrm{SD})$ & $3,91(5,73)$ & $3,32(3,82)$ \\
\hline$\Delta \mathrm{MMST} \tilde{x}$ (IQR) & $3,66(6,50)$ & $3,73(5,62)$ \\
\hline $\mathrm{p}-\mathrm{Wert}^{+}$ & $0,602^{\mathrm{c}}$ & $0,590^{\mathrm{b}}$ \\
\hline$A C E$ & C-Träger & T-Träger \\
\hline Total $(\mathrm{n}=23)$ & $17(73,9 \%)$ & $19(82,6 \%)$ \\
\hline Slow $(n=17)$ & $12(70,6 \%)$ & $13(76,5 \%)$ \\
\hline Rapid $(n=6)$ & $5(83,3 \%)$ & $6(100,0 \%)$ \\
\hline p-Wert* & $1,000^{\mathrm{a}}$ & $0,539^{\mathrm{a}}$ \\
\hline$\Delta \mathrm{MMST} \bar{x}(\mathrm{SD})$ & $4,60(5,12)$ & $4,25(5,26)$ \\
\hline$\Delta \mathrm{MMST} \tilde{x}$ (IQR) & $3,73(6,18)$ & $3,90(8,00)$ \\
\hline $\mathrm{p}$-Wert ${ }^{+}$ & $0,220^{c}$ & $0,399^{\mathrm{c}}$ \\
\hline TNK1 & A-Träger & T-Träger \\
\hline Total $(\mathrm{n}=23)$ & $18(78,3 \%)$ & $21(91,3 \%)$ \\
\hline Slow $(n=17)$ & $12(70,6 \%)$ & $15(88,2 \%)$ \\
\hline Rapid $(n=6)$ & $6(100,0 \%)$ & $6(100,0 \%)$ \\
\hline p-Wert* & $0,272^{\mathrm{a}}$ & $1,000^{\mathrm{a}}$ \\
\hline$\Delta \mathrm{MMST} \bar{x}(\mathrm{SD})$ & $4,46(5,22)$ & $4,16(5,02)$ \\
\hline$\Delta \mathrm{MMST} \tilde{x}(\mathrm{IQR})$ & $3,82(7,44)$ & $3,79(6,71)$ \\
\hline p-Wert ${ }^{+}$ & $0,234^{\mathrm{c}}$ & $0,229^{\mathrm{c}}$ \\
\hline LDLR & A-Träger & G-Träger \\
\hline Total $(n=23)$ & $14(60,9 \%)$ & $21(91,3 \%)$ \\
\hline Slow $(n=17)$ & $11(64,7 \%)$ & $16(94,1 \%)$ \\
\hline Rapid $(n=6)$ & $3(50,0 \%)$ & $5(83,3 \%)$ \\
\hline p-Wert* & $0,643^{\mathrm{a}}$ & $0,462^{a}$ \\
\hline$\Delta \mathrm{MMST} \bar{x}(\mathrm{SD})$ & $3,64(5,43)$ & $3,55(4,38)$ \\
\hline$\Delta \mathrm{MMST} \tilde{x}(\mathrm{IQR})$ & $3,04(6,48)$ & $3,73(5,62)$ \\
\hline $\mathrm{p}-\mathrm{Wert}^{+}$ & $0,746^{\mathrm{c}}$ & $1,000^{\mathrm{c}}$ \\
\hline EXOC3L2 & C-Träger & T-Träger \\
\hline Total $(\mathrm{n}=23)$ & $10(43,5 \%)$ & $23(100,0 \%)$ \\
\hline Slow $(n=17)$ & $7(41,2 \%)$ & $17(100,0 \%)$ \\
\hline Rapid $(n=6)$ & $3(50,0 \%)$ & $6(100,0 \%)$ \\
\hline p-Wert* & $1,000^{\mathrm{a}}$ & - \\
\hline$\Delta \mathrm{MMST} \bar{x}(\mathrm{SD})$ & $4,97(4,21)$ & $3,80(4,95)$ \\
\hline$\Delta \mathrm{MMST} \tilde{x}(\mathrm{IQR})$ & $3,90(3,84)$ & $3,73(6,41)$ \\
\hline p-Wert ${ }^{+}$ & $0,171^{\mathrm{c}}$ & - \\
\hline
\end{tabular}


Tabelle 18 (Fortsetzung)

\begin{tabular}{|c|c|c|}
\hline \multirow[b]{2}{*}{ CST3 } & \multicolumn{2}{|c|}{ EOAD } \\
\hline & C-Träger & T-Träger \\
\hline Total $(n=17)$ & $16(94,1 \%)$ & $5(29,4 \%)$ \\
\hline Slow $(n=13)$ & $12(92,3 \%)$ & $5(38,5 \%)$ \\
\hline Rapid (n=4) & $4(100,0 \%)$ & $0(0,0 \%)$ \\
\hline p-Wert* & $1,000^{\mathrm{a}}$ & $0,261^{\mathrm{a}}$ \\
\hline$\Delta \mathrm{MMST} \bar{x}(\mathrm{SD})$ & $3,21(2,90)$ & $0,94(2,17)$ \\
\hline$\Delta \mathrm{MMST} \tilde{x}(\mathrm{IQR})$ & $3,32(5,59)$ & $0,00(4,16)$ \\
\hline $\mathrm{p}-\mathrm{Wert}^{+}$ & $0,299^{\mathrm{b}}$ & $0,053^{\mathrm{b}}$ \\
\hline
\end{tabular}

\begin{tabular}{lcc}
\hline \multirow{2}{*}{ CST3 } & \multicolumn{2}{c}{ LOAD } \\
\cline { 2 - 3 } Total $(\mathrm{n}=23)$ & C-Träger & T-Träger \\
Slow $(\mathrm{n}=17)$ & $15(98,3 \%)$ & $11(47,8 \%)$ \\
Rapid $(\mathrm{n}=6)$ & $6(100,0 \%)$ & $3(47,1 \%)$ \\
p-Wert* $^{*}$ & $1,000^{\mathrm{a}}$ & $1,000^{\mathrm{a}}$ \\
\hline$\Delta$ MMST $^{\bar{x}}(\mathrm{SD})$ & $3,75(5,18)$ & $4,56(4,28)$ \\
$\Delta$ MMST $^{\tilde{x}}(\mathrm{IQR})$ & $3,66(7,21)$ & $3,90(5,25)$ \\
p-Wert $^{+}$ & $0,652^{\mathrm{c}}$ & $0,217^{\mathrm{c}}$ \\
\hline
\end{tabular}

DMMST: Punktverlust im MMST / Jahr (MMST-Progression)

Slow: $\triangle \mathrm{MMST} \leq 5$

Rapid: $\triangle \mathrm{MMST}>5$

* slow/rapid vs. Allelträger/Nicht-Allelträger

${ }^{+}$MMST-Progression vs. Allelträger/Nicht-Allelträger

${ }^{\mathrm{a}}$ Exakter Test nach Fisher

${ }^{\mathrm{b}}$ T-Test

${ }^{c}$ Mann-Whitney-Test

${ }^{\mathrm{d}}$ Einfaktorielle ANOVA

${ }^{\mathrm{e}}$ Kruskal-Wallis-Test

\subsubsection{PICALM}

\section{$\underline{\text { EOAD }}$}

C-Träger und Nicht-C-Träger bzw. T-Träger und Nicht-T-Träger unterschieden sich weder in der Häufigkeit der langsam und schnell verlaufenden Fälle noch in der MMST-Progression in signifikanter Weise (siehe Tabelle 18).

\section{$\underline{\mathrm{LOAD}}$}

Hinsichtlich der Anzahl der langsamen und schnellen Fälle ergab sich zwischen C-Trägern und Nicht-C-Trägern sowie zwischen G-Trägern und Nicht-G-Trägern kein deutlicher Unterschied (siehe Tabelle 18). Bei C-Trägern konnte mit einem Mittelwert von 5,9 Punkten (SD 5,8) jedoch eine deutlich höhere MMST-Progression als bei Nicht-C-Trägern $(\bar{x}=1,9$ Punkte, SD 3,3) beobachtet werden. Dieser Unterschied war allerdings nicht statistisch signifikant ( $p=0,055$, T-Test, Abb. 19). G-Träger und Nicht-G-Träger unterschieden sich in ihrer MMST-Progression nicht deutlich (siehe Tabelle 18). 


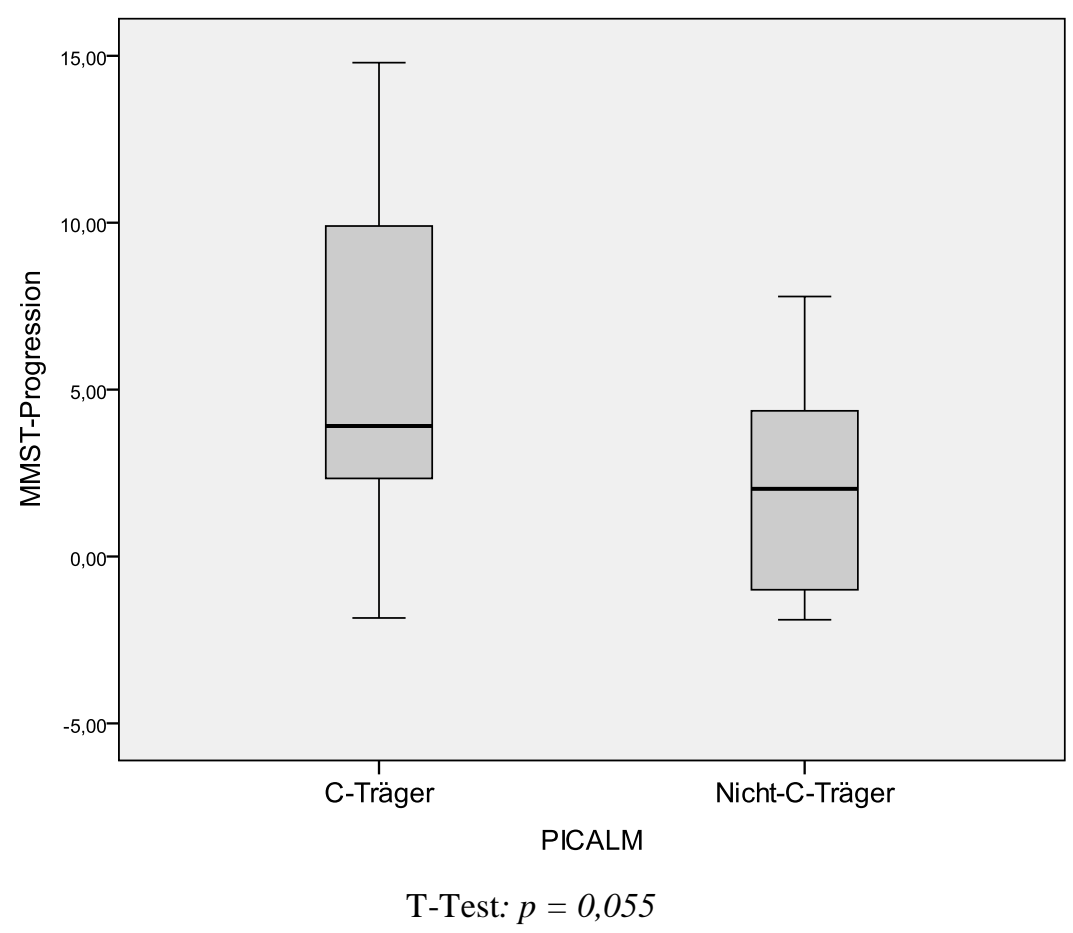

Abb. 19: PICALM: MMST-Progression von C-Trägern und Nicht-C-Trägern bei Patienten mit spätem Krankheitsbeginn

\subsubsection{CST3}

\section{$\underline{\text { EOAD }}$}

Für C- und Nicht-C-Träger bzw. T- und Nicht-T-Träger konnte kein signifikanter Unterschied in der Anzahl der schnell und langsam verlaufenden Fälle (,slow“ vs. „,rapid“) festgestellt werden. C-Träger und Nicht-C-Träger unterschieden sich zudem nicht in ihrer MMSTProgression (siehe Tabelle 18). T-Träger wiesen jedoch mit durchschnittlich 0,9 Punkten (SD 2,2) eine deutlich geringere MMST-Progression als Nicht-T-Träger auf ( $\bar{x}=3,9$ Punkte, SD 2,8; Abb. 20). Dieser Unterschied war nah an der Grenze zur statistischen Signifikanz ( $p=$ 0,053, T-Test). 


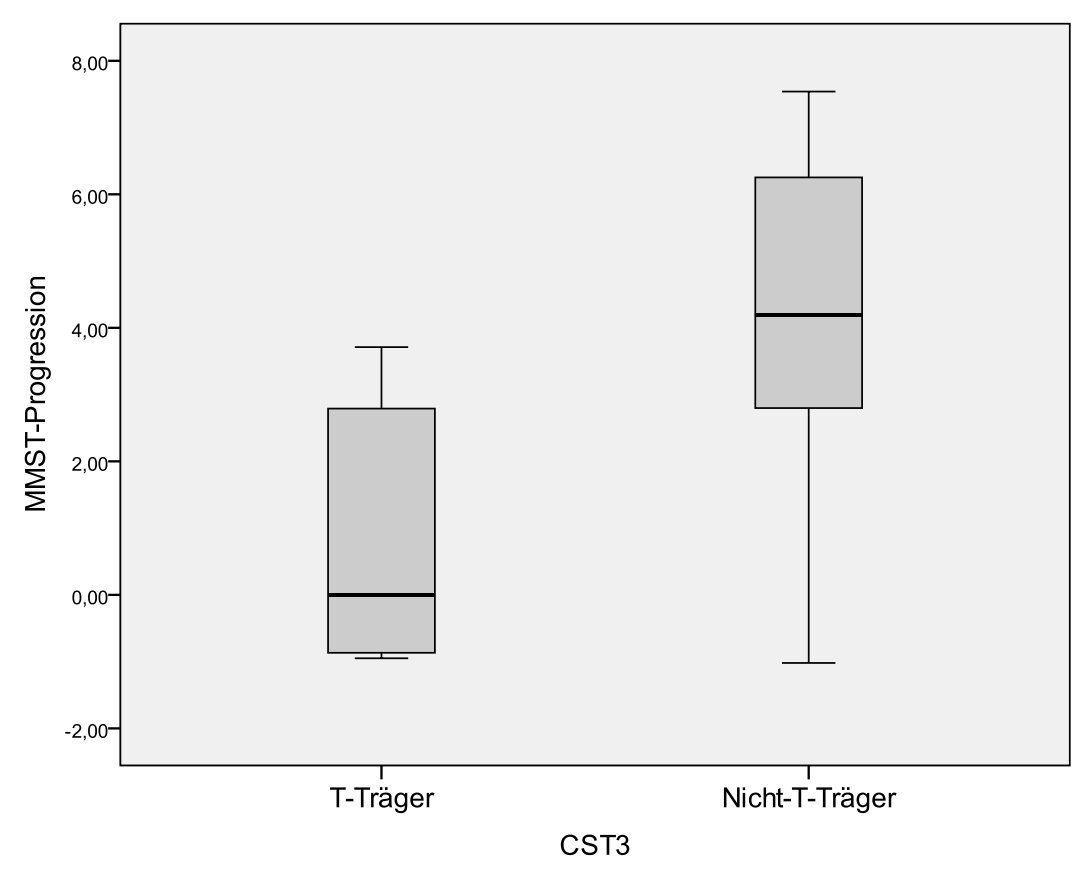

T-Test: $p=0,053$

Abb. 20: CST3: MMST-Progression von T-Trägern und Nicht-T-Trägern bei Patienten mit frühem Krankheitsbeginn

\section{$\underline{\mathrm{LOAD}}$}

Hier zeigten sich zwischen C-Trägern und Nicht-C-Trägern bzw. zwischen T-Trägern und Nicht-T-Trägern weder hinsichtlich der Häufigkeit von langsam und schnell verlaufenden Fällen noch hinsichtlich der MMST-Progression deutliche Unterschiede (siehe Tabelle 18). 


\section{Diskussion}

\subsection{Studiendesign und Methodik}

In der vorliegenden longitudinalen Studie wurden 11 Polymorphismen, für die in GWAStudien eine Assoziation mit der AD nachgewiesen werden konnte, hinsichtlich ihres Einflusses auf die Progressionsgeschwindigkeit in einem Kollektiv von 42 Patienten mit AD untersucht. Im Folgenden sollen die Ergebnisse der Studie und die verwendeten Methoden benannt und kritisch diskutiert werden.

Die größte Limitation der vorliegenden Arbeit stellt die kleine Stichprobenzahl von 42 Studienteilnehmern dar, so dass lediglich 10 Probanden der rapid-verlaufenden Gruppe (MMST > 5 Punkte/Jahr) zugeordnet werden konnten. Die Verteilung der Teilnehmer auf die verschiedenen Genotypen sowie die Stratifikation nach Geschlecht und Krankheitsbeginn führten zu einer weiteren Verkleinerung der Patientenanzahl in den jeweiligen Untergruppen. Es muss also davon ausgegangen werden, dass allein aufgrund der kleinen Stichprobenanzahl keine validen Aussagen über die Assoziation der untersuchten Polymorphismen mit einem veränderten Krankheitsverlauf getroffen werden können. In diesem Zusammenhang sollte allerdings berücksichtigt werden, dass es sich durch die bevorzugte Rekrutierung von rapiden Demenzen um ein sehr spezielles Patientenkollektiv handelt, welches im Vergleich zu klassischen AD-Kohorten einen höheren Anteil an schnell verlaufenden AD-Fällen aufweist. Diese beabsichtigte „Verzerrung“ sollte eine genauere Charakterisierung der rapidprogressiven AD ermöglichen und durch eine hohe Varianz an Progressionsgeschwindigkeiten die Identifikation von damit assoziierten Polymorphismen erleichtern. Die sehr kleine Fallzahl ist vor allem dem Umstand geschuldet, dass die rapidprogressive Form der AD eine sehr seltene Subgruppe darstellt und dass die vorliegende Arbeit lediglich auf Daten der ersten zwei Rekrutierungsjahre eines Forschungsprojektes zurückgreift, welches auf viele Jahre ausgelegt ist. Es ist also damit zu rechnen, dass durch die Rekrutierung weiterer Patienten in den folgenden Jahren möglicherweise robustere Aussagen getroffen werden können. Um dem Nachteil einer aktuell geringen Probandenzahl trotzdem entgegenzuwirken, wurde die Studienpopulation mithilfe strikter Diagnosekriterien so präzise wie möglich charakterisiert. Dazu wurden für den Einschluss von Patienten die Forschungskriterien nach Dubois (Dubois et al. 2007) verwendet, welche im Gegensatz zu den klassischen NINCDS-ADRDA-Kriterien (McKhann et al. 1984) neben neuropsychologischen Aspekten auch MRT-Befunde und Liquormarker beinhalten. Somit 
konnte eine hohe diagnostische Verlässlichkeit ermöglicht werden. Bei den nach Geschlecht und Krankheitsbeginn stratifizierten Daten wurden zudem lediglich die entsprechenden Allelträger und Nicht-Allelträger verglichen, um die Gruppen nicht weiter zu verkleinern. Hinsichtlich der geringen Patientenanzahl sei zudem erwähnt, dass einige frühere genetische Studien, z.B. zum ApoE-Gen (Corder et al. 1993; Strittmatter et al. 1993), auch mit kleineren Kohorten wichtige Ansatzpunkte aufzeigen konnten, die schließlich in größeren Studien bestätigt werden konnten. Die Intention der vorliegenden Studie war es somit, als eine Art „Pilotstudie“ lediglich eine eventuelle Richtung vorzugeben, die in weitergehenden Forschungsbemühungen verfolgt werden kann.

Neben der kleinen Teilnehmerzahl muss zudem darauf hingewiesen werden, dass in der aktuellen Arbeit hinsichtlich der Untersuchung mehrerer Polymorphismen keine Korrektur für multiples Testen durchgeführt wurde. Die nominal signifikanten Ergebnisse insbesondere von PICALM, BIN1 und TNK1 halten einer solchen Korrektur nicht stand. Einen weiteren Hinweis auf einen möglichen Falschbefund gibt die Tatsache, dass bei keinem der Polymorphismen ein monotoner Trend hinsichtlich der MMST-Progression mit steigender Risikoallelzahl zu beobachten war. Dies wäre unter der Annahme eines Alleldosiseffektes jedoch notwendig.

Zur Messung des Progresses wurde der Punktverlust im MMST innerhalb eines Jahres verwendet. Aus praktischen Gründen wurde dabei in der aktuellen Arbeit von einem linearen Verfall ausgegangen, d.h. es wurde angenommen, dass die Progressionsgeschwindigkeit im gesamten Verlauf der Erkrankung gleich ist. Es muss jedoch berücksichtigt werden, dass für die AD unterschiedliche, auch nicht-lineare Verlaufstypen beschrieben sind (Wilkosz et al. 2010). Um diese verschiedenen Verläufe besser zu erfassen und zu berücksichtigen, wären entsprechend längere Zeitintervalle nötig.

Bei der Interpretation möglicher Assoziationen zwischen Polymorphismen und der Progressionsgeschwindigkeit muss außerdem berücksichtigt werden, dass Aspekte wie Umwelteinflüsse, Medikation, Komorbiditäten, unterschiedliche kognitive Reserven oder auch Interaktionen der Polymorphismen untereinander den Verlauf der Erkrankung beeinflussen können. Auch sollte in Erwägung gezogen werden, dass unterschiedlichen Verläufen der $\mathrm{AD}$ womöglich eine neuropathologische Heterogenität zugrundeliegen kann (Murray et al. 2011). Wilkosz et al. beschrieben in einer longitudinalen Kohortenstudie zudem unterschiedliche Verlaufstypen (Trajektorien) der AD, die sich am besten anhand des Alters und des initialen MMST der Probanden definieren ließen. Darüber hinaus zeigten Patienten 
mit psychotischen Symptomen in allen Trajektorien einen schnelleren Verlauf (Wilkosz et al. 2010).

Der MMST an sich bietet einen gut validierten Parameter für die Krankheitsprogression (Soubelet und Salthouse 2011). Eine genaue Abbildung der Verschlechterung in einzelnen kognitiven Domänen ist jedoch durch Summation der in den Einzelaufgaben erreichten Punkte nicht möglich. Einflüsse von Polymorphismen auf bestimmte kognitive Domänen konnten daher nicht erfasst werden.

Für die neuropsychologische Diagnostik der AD wurde in dieser Arbeit die CERADTestbatterie verwendet (Morris et al. 1988; Morris et al. 1989). Für den amerikanischen CERAD konnte eine gute Objektivität, Reliabilität und Validität nachgewiesen werden (Morris et al. 1988; Morris et al. 1989). Die deutschsprachige Version der CERADTestbatterie wurde von der Geriatrischen Universitätsklinik Basel an einer Stichprobe von 614 gesunden Patienten normiert und im Rahmen einer Multicenterstudie validiert (Thalmann et al. 2000; Aebi 2002).

Die im Rahmen der Diagnostikkriterien nach Dubois ebenfalls verwendeten Liquormarker beinhalteten Tau, Phospho-Tau und $A \beta_{1-42}$. Für Tau konnte anhand einer Analyse von 36 Studien mit 2500 Patienten und 1400 Kontrollen eine Sensitivität von $81 \%$ und Spezifität von 90\% ermittelt werden (Blennow und Hampel 2003). Die aus 11 Studien mit 800 Patienten und 370 Kontrollen berechneten Werte für Sensitivität und Spezifität betrugen für Phospho-Tau 80\% und 92\% (Blennow und Hampel 2003). Für $A \beta_{1-42}$ ergaben sich anhand einer Analyse von 13 Studien mit 600 Patienten und 450 Kontrollen eine Sensitivität von $86 \%$ und eine Spezifität von 90\% (Blennow und Hampel 2003). Durch Kombination von Liquormarkern können noch höhere Sensitivitäten und Spezifitäten erreicht werden (Blennow und Hampel 2003).

Bei allen Polymorphismen wurde das Vorliegen des Hardy-Weinberg-Gleichgewichts (HWG) als Qualitätsmarker für die Genotypisierung überprüft. Kein Polymorphismus verletzte das HWG, so dass systematische Fehler bei der Genotypen-Bestimmung weitestgehend ausgeschlossen werden können.

\subsection{Soziodemographische Daten und kognitiver Status}

\subsubsection{Alter und Geschlecht}

Mit einem Durchschnittsalter von 67 Jahren und einer Altersspanne von 49 bis 86 Jahren waren die Studienpatienten deutlich jünger als in Prävalenzstudien von demenziellen 
Erkrankungen in der BRD beschrieben (Bickel 2005; Ziegler und Doblhammer 2009). Dies ist damit zu begründen, dass die meisten Studienteilnehmer ursprünglich unter dem Verdacht einer Prionerkrankung, bei welcher der Erkrankungsgipfel innerhalb der siebten Lebensdekade liegt (Heinemann et al. 2007), an das NRZ gemeldet wurden. Dies ist auch eine Erklärung dafür, dass der Anteil an Patienten mit EOAD im vorliegenden Patientenkollektiv mit rund $40 \%$ wesentlich höher als in klassischen Kohorten liegt. In der Literatur wurde im Jahre 2002 die Absolutzahl der präsenilen AD-Fälle in Deutschland auf lediglich 10000 geschätzt (Bickel 2002).

Der mit 62\% deutlich höhere Anteil von Frauen im Kollektiv entspricht der Beobachtung aus epidemiologischen Studien, in denen Frauen ca. 70\% aller Demenzfälle ausmachen (Bickel 2005; Ziegler und Doblhammer 2009). In diesen Studien waren Männer nur in der Altersklasse unter 70 Jahren etwas häufiger von einem Demenzleiden betroffen. Die Beobachtungen der vorliegenden Arbeit, dass 59\% der EOAD-Fälle männlich und 76\% der LOAD-Fälle weiblich waren, sind demnach mit den Literaturergebnissen zu vereinbaren. Insgesamt waren bei den männlichen Studienteilnehmern rund 63\% jünger als 65 Jahre, bei den Frauen 27\%. Dennoch wiesen auch die weiblichen Probanden mit 69 Jahren ein deutlich niedrigeres Durchschnittsalter als bei klassischen AD-Kohorten auf (Durchschnittsalter der Männer: 64 Jahre).

Durch das besondere Rekrutierungverfahren über das NRZ handelt es sich hier also um ein spezielles Kollektiv mit im Vergleich zur klassischen AD relativ jungen Patienten.

\subsubsection{Krankheitsdauer}

Die Zeit vom Beginn der ersten Symptome bis zur Erstuntersuchung betrug rund 17 Monate (Median). Die bisherige Krankheitsdauer bei den weiblichen Studienteilnehmern war mit einem Median von rund 15 Monaten etwa halb so lang wie bei den Männern $(\tilde{x}=30$ Monate). Hier muss bedacht werden, dass es sich um subjektive Schätzungen von Angehörigen der Patienten handelt. Da aufgrund einer guten Fassade von AD-Patienten die Erkrankung auch für nahe Angehörige zunächst unentdeckt bleiben kann, könnte diese Schätzung möglicherweise zu gering ausgefallen sein. Es ist ebenfalls zu berücksichtigen, dass es sich bei der Erstuntersuchung im Rahmen der rpAD-Studie nicht zwingend um die erste Konsultation eines Arztes gehandelt haben muss und somit auch schon früher die Diagnose einer AD gestellt worden sein kann. 


\subsubsection{MMST bei Erst- und Zweituntersuchung}

Der Median der MMST-Scores aller Studienpatienten bei Erstuntersuchung lag bei 21,5 Punkten (IQR = 9,0). Hierbei fiel auf, dass die weiblichen Teilnehmer mit einem Median von 16,5 Punkten $(\mathrm{IQR}=11,0)$ signifikant schlechter abschnitten als die männlichen Patienten $(\tilde{x}=24,0$ Punkte, IQR $=5,5)$. Wenn man die Einteilung des NICE-SCIE (The National Institute for Health and Clinical Excellence and the Social Care Institute for Excellence) (NICE-SCIE 2007) anhand des MMST zugrunde legt, befanden sich die Frauen somit bereits im mittleren Stadium der Erkrankung, während die Männer dem frühen Stadium zugeordnet werden konnten. Dies ist insofern interessant, als dass die geschätzte bisherige Krankheitsdauer bei den Frauen nur halb so lang war wie bei den Männern (siehe 4.2.2). Die Tatsache, dass sich im Verlauf der vorliegenden Studie anhand des MMST-Verfalles bei Frauen eine objektiv langsamere Progression als bei Männern zeigte, lässt darauf schließen, dass die Schätzungen der Angehörigen bezüglich der Krankheitsdauer möglicherweise zu ungenau waren (siehe 4.2.4).

Die Spannweite des MMST bei Erstuntersuchung reichte von 2 bis 29 Punkte. Werte über 25 Punkte sind in bestimmten Klassifikationen zwar mit einem MCI vereinbar, jedoch noch nicht mit einer AD. Für den Studieneinschluss in der vorliegenden Arbeit war jedoch die revidierte Fassung der NINCDS-ADRDA-Kriterien nach Dubois et al. maßgeblich (Dubois et al. 2007, siehe Anhang, Tabelle 23, S. 81). Die strikten Kriterien für eine wahrscheinliche AD, die neben den neuropsychologischen Aspekten auch Biomarker bzw. MRT-Befunde berücksichtigen, wurden von allen Patienten erfüllt. Insbesondere die für die Diagnosestellung als Kernsymptom notwendige frühe und signifikante Minderleistung des episodischen Gedächtnisses konnte in der CERAD-Testung bei allen Studienteilnehmern nachgewiesen werden.

\subsubsection{MMST-Progression}

Der Median der MMST-Progression aller Studienteilnehmer lag bei 3,7 Punkten (IQR = 5,3). Dieser Wert ist etwas höher als in der Literatur, in der durchschnittliche Progressionsraten von 3 Punkten für die AD angegeben werden (Folstein et al. 1975; Morris et al. 1993). Zu berücksichtigen ist zudem, dass es sich bei letzteren Angaben um Beobachtungen des natürlichen Verlaufes einer AD, d.h. ohne medikamentöse antidementive Therapie, handelt. Im vorliegenden Kollektiv erhielten ca. $91 \%$ der Patienten während des Untersuchungszeitraumes eine medikamentöse Behandlung mit einem Cholinesterasehemmer 
und/oder einem Glutamat-Antagonisten. Verglichen mit der REAL.FR-Kohortenstudie (Cortes et al. 2005), in der annähernd 90\% der Teilnehmer eine anticholinerge Therapie erhielten und eine durchschnittliche MMST-Progression von 2 Punkten im ersten Follow-upJahr (2,5 Punkte im zweiten Jahr) beobachtet werden konnte, weist das vorliegende Kollektiv demnach eine deutlich schnellere Progression auf.

In der vorliegenden Arbeit wurden Patienten, die mehr als 5 Punkte pro Jahr im MMST verloren, als ,,rapid“ bezeichnet. Diese Einteilung beruht auf dem Vorschlag einiger Autoren hinsichtlich einer Definition der rpAD (Doody et al. 2001; Wallin et al. 2010). Zudem entsprach dieser Wert in etwa dem 75. Perzentil der MMST-Progression aller Patienten im Kollektiv. Die als rapid klassifizierten Patienten (MMST-Progression > 5 Punkte) machten einen Anteil von 24\% aus. Ob dieser Anteil ungefähr die Situation in der Gesamtbevölkerung widerspiegelt, ist bislang noch unklar, da Vergleichsstudien unterschiedliche Definitionen für den Begriff rapid verwenden. Die Häufigkeit schwankt dementsprechend überwiegend zwischen 10 und 30\% (Dumont et al. 2005; Carcaillon et al. 2007; Cortes et al. 2008; Soto et al. 2008b; Soto et al. 2008a; Wallin et al. 2010; Schmidt et al. 2011).

Frauen wiesen mit einem Median von 2,0 Punkten (IQR = 5,9) eine deutlich langsamere MMST-Progression auf als die Männer ( $\tilde{x}=4,0, \mathrm{IQR}=2,8)$. Dies widerspricht der scheinbar erhöhten Progression der weiblichen Patienten bis zur Erstuntersuchung, die wahrscheinlich auf ungenaue Schätzungen der Angehörigen zurückgeführt werden kann. Hinsichtlich der Gruppenzuteilung „slow/rapid“ wurden unter den männlichen Studienteilnehmern jedoch nicht deutlich mehr schnell verlaufende Fälle als bei den weiblichen Patienten gefunden. Der Zeitpunkt des Krankheitsbeginns (EOAD/LOAD), das Alter bei Erstuntersuchung, der Ausbildungsgrad sowie die Ausgangspunktzahl im MMST und die bisherige Krankheitsdauer schienen keinen deutlichen Einfluss auf die Progression bzw. auf die Zuordnung zur „langsamen“ oder „rapiden“ Gruppe zu haben und konnten somit als starke Störfaktoren ausgeschlossen werden.

\subsection{Genetische Polymorphismen}

\subsubsection{PICALM}

PICALM (Phosphatidylinositol-binding clathrin assembly protein) kodiert ein gleichnamiges, vor allem in Neuronen exprimiertes Protein, welches eine Rolle in der Clathrin-vermittelten Endozytose und im intrazelluären Membrantransport spielt. Die Verbindung zur AD wird darin gesehen, dass PICALM unter anderem auch in den Membrantransport des Proteins 
VAMP2 involviert ist. Letzteres ist notwendig für die Neurotransmitterfreisetzung an präsynaptischen Membranen und somit wichtig für Nervenfunktion und Gedächtnis (Harold et al. 2009; Sleegers et al. 2009). Weiterhin wird vermutet, dass PICALM das Risiko für eine $\mathrm{AD}$ vielleicht auch durch eine Beeinflussung der Endozytose von APP und somit dessen Prozessierung modulieren könnte (Harold et al. 2009). Baig et al. beschreiben hingegen eine bevorzugte Expression von PICALM im Endothel von zerebralen Blutgefäßen und legen somit eine Rolle in der A $\beta$-Clearance nahe (Baig et al. 2010).

In der vorliegenden Arbeit ließ sich für den untersuchten rs541458-Polymorphismus des PICALM-Gens bei C-Trägern eine signifikant höhere durchschnittliche MMST-Progression als bei Nicht-C-Trägern nachweisen $(p=0,039)$. Die einzelnen Genotypen CC, TC und TT unterschieden sich jedoch nicht signifikant $(p=0,119)$. Zudem ließ sich kein Trend zu einer schnelleren Progression bei steigender Anzahl von C-Allelen im Sinne eines Alleldosiseffektes erkennen, so dass die Möglichkeit eines Falschbefundes nicht auszuschließen ist. In den Gruppen „,slow“ und „,rapid“ gab es keine deutlichen Unterschiede hinsichtlich der Genotyp- oder Allelverteilung, so dass sich hier keine Prädisposition zu einer rpAD erkennen ließ.

Nach Stratifikation in EOAD und LOAD konnte auch bei C-Allel-Trägern mit LOAD eine deutlich höhere MMST-Progression als bei Nicht-C-Trägern mit LOAD beobachtet werden. Dieser Unterschied befand sich an der Grenze zur statistischen Signifikanz $(p=0,055)$. Bei Studienteilnehmern mit EOAD zeigte sich diesbezüglich kein deutlicher Unterschied, so dass unter dem Vorbehalt eines möglichen Falschbefundes (s.o.) spekuliert werden könnte, dass ein progressions-beschleunigender Effekt des C-Allels womöglich bevorzugt Patienten vom Spättyp der AD betreffen könnte.

In der Stratifikation nach Geschlecht konnte das im Gesamtkollektiv beobachtete Ergebnis nicht nachgewiesen werden. Weder in der Einzelgruppe der Frauen noch der Männer zeigte das C-Allel einen signifikanten Effekt auf die Progressionsgeschwindigkeit. Diese fehlende Signifikanz ist eventuell auch auf die geringe Größe der Untergruppen zurückzuführen.

Die Ergebnisse lassen zusammenfassend zunächst vermuten, dass das Vorhandensein eines CAllels mit einer erhöhten Progressionsgeschwindigkeit assoziiert ist, wobei ein vermehrtes Auftreten einer rpAD bei C-Trägern nicht beobachtet werden konnte. Aufgrund des fehlenden monotonen Trends mit steigender Allelzahl sowie vor dem Hintergrund der sehr kleinen Stichprobenanzahl der Studie muss jedoch eher davon ausgegangen werden, dass auf der Basis der vorliegenden Daten keine validen Aussagen über eine mögliche Assoziation getroffen werden können. 
Nach Metaanalysen der Alzgene-Datenbank ist das C-Allel des gewählten PICALMPolymorphismus (rs541458) mit einem niedrigeren Risiko für eine $\mathrm{AD}$ assoziiert (OR 0,88$)$ (Bertram et al. 2007). Dass ein Prädispositionsfaktor jedoch nicht zwingend zu einer schnelleren Progression führen muss, kann anhand des ApoE-Gens gezeigt werden, für welches in der Literatur durchaus widersprüchliche Ergebnisse in Bezug auf die Progressionsgeschwindigkeit gefunden werden können (siehe 4.3.4.5). Für den untersuchten PICALM-Polymorphismus gibt es in der Literatur bislang keine Angaben über eine mögliche Beeinflussung der Krankheitsprogression. $\mathrm{Hu}$ et al. untersuchten jedoch einen möglichen Effekt des rs3851179-Polymorphismus auf die Progressionsgeschwindigkeit, die mithilfe der Clinical Dementia Rating Scale $(C D R)$ bei 597 AD-Patienten gemessen wurde. Hierbei konnte für den TC-Genotyp ein signifikant höherer Verfall als für TT und CC gezeigt werden (Hu X et al. 2011).

Anhand der Ergebnisse der vorliegenden Arbeit für PICALM kann lediglich die Vermutung geäußert werden, dass die beschriebenen pathophysiologischen Vorgänge möglicherweise auch Bedeutung für die Progressionsgeschwindigkeit der AD haben. In weitergehenden Studien mit deutlich größeren Kollektiven muss daher überprüft werden, ob die hier dargestellten Ergebnisse repliziert werden können.

\subsubsection{BIN1}

Das BIN1-Gen (Bridging Integrator 1) befindet sich auf dem langen Arm von Chromosom 2. Es kodiert ein gleichnamiges Protein, welches zur Gruppe der Amphiphysine gehört und am häufigsten in Muskel und Gehirn exprimiert wird (Wechsler-Reya et al. 1997). Amphiphysine scheinen unter anderem eine wichtige Rolle in der neuronalen Membranorganisation und der Endozytose in Synapsen zu spielen (Wigge et al. 1997; Wigge und McMahon 1998). Für Knockout-Mäuse mit verringerter Amphiphysin-Expression konnten Störungen des synaptischen Vesikelrecyclings sowie kognitive Defizite gezeigt werden (Di Paolo et al. 2002). Es wird vermutet, dass das BINI-Gen im Rahmen der Endozytoseprozesse möglicherweise ebenfalls den APP-Metabolismus und damit die Entstehung der AD beeinflussen könnte (Lambert und Amouyel 2011). Die Alzgene-Datenbank beschreibt für den im Rahmen der aktuellen Arbeit untersuchten rs744373-Polymorphismus von BIN1 eine Odds Ratio von 1,17 für das G-Allel hinsichtlich des Risikos einer AD (Bertram et al. 2007). Die einzige Studie, die eine Assoziation zwischen einem BIN1-Polymorphismus (rs12989701) 
und der Progressionsgeschwindigkeit der AD untersuchte, erbrachte ein negatives Ergebnis (Hu X et al. 2011).

Beim in der vorliegenden Arbeit untersuchten BIN1-Polymorphismus (rs744373) war der Genotyp GG mit einer deutlich höheren MMST-Progression verbunden als AA und GA. Jedoch erwies sich dieser Unterschied weder im Vergleich der drei Genotypen untereinander $(p=0,191)$ noch im Vergleich zwischen A-Trägern (AA und GA) und Nicht A-Trägern (GG, $p=0,114)$ als statistisch signifikant. Zudem ließ sich kein monotoner Trend für die MMSTProgression mit steigender Allelzahl nachweisen. In der separaten Analyse von weiblichen und männlichen Individuen zeigten auch Frauen ohne A-Allel (Genotyp GG) eine deutlich höhere MMST-Progression als die A-Trägerinnen. Dieser sich bereits im Gesamtkollektiv andeutende Unterschied lag hier an der Grenze zur statistischen Signifikanz $(p=0,056)$. Ein Vergleich mit den männlichen Studienteilnehmern war nicht möglich, da diese im untersuchten BIN1-Polymorphismus alle ein A-Allel aufwiesen. Eine Aussage darüber, ob sich das weibliche Geschlecht und der BIN1-Polymorphismus in Bezug auf die Progressionsgeschwindigkeit möglicherweise gegenseitig beeinflussen, kann aufgrund der geringen Teilnehmerzahl daher nicht getroffen werden. In der Stratifikation nach Krankheitsbeginn (EOAD vs. LOAD) ließ sich zwischen A-Trägern und Nicht-A-Trägern kein deutlicher Unterschied zeigen.

Hinsichtlich der Zuordnung zu den Gruppen „slow“ und „rapid“ konnte zwischen den einzelnen BIN1-Genotypen ein signifikanter Unterschied beobachtet werden ( $p=0,037)$. Der Genotyp AG war im Vergleich zu den Genotypen AA/GG signifikant häufiger in der langsam verlaufenden Gruppe nachweisbar $(p=0,026)$. Beim Vergleich der Häufigkeiten von ATrägern und Nicht-A-Trägern bzw. G-Trägern und Nicht-G-Trägern in den zwei Gruppen zeigte sich jedoch keine deutliche Differenz, so dass hier kein Muster im Sinne eines Gendosiseffekts oder einer Allelabhängigkeit beobachtet werden konnte. Gleiches gilt für die Stratifikation nach Geschlecht und Krankheitsbeginn.

Zusammenfassend lassen die Ergebnisse zu BINl keine eindeutige Interpretation zu. Die Werte könnten Anlass zur Vermutung geben, dass das A-Allel eher mit einem langsameren Verlauf bzw. das G-Allel (bei homozygoten Trägern) mit einem schnelleren Verlauf assoziiert sein könnte. Letzteres ließe eventuell Raum zur Spekulation, dass das G-Allel womöglich nicht nur zur Krankheit selbst sondern auch zu einer schnelleren Progression prädisponieren könnte. Unter Zusammenschau der vorliegenden Daten zu BIN1 liegt jedoch die Schlussfolgerung sehr nah, dass die Ergebnisse aufgrund der Inkonstanz der Werte sowie vor dem Hintergrund des fehlenden Alleldosiseffekts und insbesondere der geringen 
Probandenzahl keine robusten Aussagen zu einer möglichen Assoziation zulassen. Hier sind Studien mit weitaus größeren Teilnehmerzahlen nötig, um einen eventuellen Effekt zu verdeutlichen.

\subsubsection{TNK1}

TNK1 ist eine Non-Receptor-Thyrosinkinase, für die eine Assoziation mit dem Enzym Phospholipase C (PLC) gamma 1 beschrieben ist und die somit vermutlich eine Rolle in der zellulären Phospholipid-Signaltransduktion spielt (Felschow et al. 2000). Die PLC spaltet Phosphatidyl-Inositol-4,5-biphosphat in die beiden sekundären Botenstoffe Diacylglycerin (DAG) und Inositol-1,4,5-Triphosphat $\left(\mathrm{IP}_{3}\right)$, ein Mechanismus, welcher u.a. bei muskarinergen Acetylcholinrezeptoren zum Tragen kommt. Eine veränderte Regulation der PLC-Aktivität konnte auch in Gehirnen von Patienten mit AD nachgewiesen werden (Crews et al. 1994; Jope et al. 1994; Greenwood et al. 1995). Zudem ließ sich eine Beeinflussung der APP-Prozessierung und der Amyloidproduktion durch Stimulierung PLC-gekoppelter Rezeptoren zeigen (Emmerling et al. 1993; Wolf et al. 1995). Es wird daher vermutet, dass eine veränderte TNK1-Aktivität über eine Beeinflussung der PLC zur Alzheimer-Pathologie beitragen könnte (Grupe et al. 2007). Untersuchungen über die Auswirkung einer Aktivitätsänderung von TNK1 auf die Progressionsgeschwindigkeit der AD liegen bislang nicht vor.

In der aktuellen Arbeit konnte für den rs1554948-Polymorphismus von TNK1 ein deutlicher Unterschied in den MMST-Progressionswerten der einzelnen Genotypen beobachtet werden, welcher jedoch nicht statistisch signifikant war $(p=0,086)$. Die höchste Progressionsgeschwindigkeit wies dabei der Genotyp AT auf, gefolgt von TT und mit deutlichem Abstand AA. T-Träger boten einen deutlich schnelleren Verlauf als Nicht-TTräger (Genotyp AA), eine strikte Allelabhängigkeit für das T-Allel ließ sich durch statistische Tests jedoch nicht untermauern $(p=0,121)$. Zwischen langsam und schnell verlaufenden Fällen (,,slow“ vs. ,,rapid“) zeigte sich kein deutlicher Unterschied in der Alleloder Genotypverteilung.

Bei der Stratifikation nach Geschlecht und Krankheitsbeginn ließen sich für die einzelnen Allelträger weder in der absoluten MMST-Progression noch in der Verteilung auf die Gruppen ,, slow“ und „rapid“ deutliche Unterschiede feststellen.

Zusammenfassend lassen die Ergebnisse für TNK1 keinen eindeutigen Schluss zu. Ein Effekt im Sinne einer Allelabhängigkeit konnte lediglich für das T-Allel schwach angedeutet 
werden, dessen Träger eine schnellere MMST-Progression als Nicht-T-Träger aufwiesen. Ein konsistenter monotoner Trend mit steigender Allelzahl ergab sich allerdings nicht. Die Tatsache, dass der Genotyp AA mit einer deutlich langsameren Progression assoziiert war, ließe andererseits Raum zur Spekulation, dass der homozygote Besitz des A-Allels möglicherweise vor einem schnellen Krankheitsverlauf schützen könnte. Eine Prädisposition eines Genotyps bzw. eines Allels für einen schnellen Verlauf im Sinne der Defintion einer rpAD ließ sich anhand der Ergebnisse nicht feststellen. Insgesamt muss unter zusätzlicher Berücksichtigung der geringen Stichprobenzahl der Studie allerdings davon ausgegangen werden, dass die aktuellen Daten für TNK1 nicht ausreichen, um eine tragfähige Aussage hinsichtlich einer Assoziation des Polymorphismus treffen zu können.

In der Metaanalyse der Alzgene-Datenbank hinsichtlich des Risikos der Entwicklung einer AD war das A-Allel des untersuchten TNK1-Polymorphismus mit einer protektiven Funktion assoziiert (OR 0,84) (Bertram et al. 2007). Vor dem Hintergrund der aktuellen Ergebnisse könnte also diskutiert werden, ob das A-Allel möglicherweise nicht nur für die Entstehung einen protektiven Effekt, sondern auch auf den Verlauf der AD einen verlangsamenden Einfluss hat. Aufgrund der oben beschriebenen Limitationen sind jedoch zukünftige, deutlich größere Studien nötig, um den diskreten Effekt der vorliegenden Arbeit möglicherweise zu verdeutlichen.

\subsubsection{Weitere Polymorphismen}

\subsubsection{GWA14q32.13}

Über das GWA14q32.13-Gen liegen bislang nur wenige Informationen vor. Auch das von diesem kodierte Protein und dessen Funktion sind bisher unbekannt. Der Genlokus liegt in räumlicher Nähe zum SERPINA13-Gen, welches zum alphal-Antiproteinase-Gencluster gehört (Grupe et al. 2007). Diese Genfamilie scheint eine Rolle in Störungen der Proteinkonformation wie dem alpha-1-Antitrypsinmangel zu spielen (Carrell und Lomas 2002). Eine Metaanalyse von Alzgene ergab für den in der vorliegenden Arbeit untersuchten rs11622883-Polymorphismus eine Odds Ratio von 0,84 (A vs. T) (Bertram et al. 2007). Untersuchungen über eine mögliche Assoziation dieses Polymorphismus mit der Progressionsgeschwindigkeit der AD liegen bislang nicht vor.

In der aktuellen Arbeit zeigten Männer, die im untersuchten GWA14q32.13-Polymorphismus ein T-Allel aufwiesen, eine signifikant niedrigere MMST-Progression als der einzige Nicht-TTräger $(p=0,001)$. Dieser Unterschied konnte weder im Gesamtkollektiv noch bei den 
weiblichen Studienteilnehmern gesehen werden. Die Aussagekraft des Ergebnisses ist jedoch als sehr eingeschränkt zu bewerten, da sich lediglich ein männlicher Nicht-T-Träger unter den Probanden befand. Eine Aussage über eine mögliche Wechselwirkung zwischen dem männlichem Geschlecht und dem GWA14q32.13-Polymorphismus kann daher anhand dieser Studie nicht getroffen werden.

\subsubsection{ACE}

Das ACE-Gen kodiert eine gleichnamige Peptidase, welche an der Konversion von Angiotensin I in das vasoaktive Angiotensin II beteiligt ist. Das Enzym kommt sowohl membrangebunden als auch als lösliche Variante vor (Vardy et al. 2009). Eine Verbindung zur AD wird vor allem durch die Beobachtung gesehen, dass ACE dosisabhängig imstande ist, das neurotoxische $\beta$-Amyloid $\mathrm{zu}$ degradieren und somit einer Aggregation entgegenzuwirken ( $\mathrm{Hu} \mathrm{J}$ et al. 2001).

In der vorliegenden Arbeit wurde der rs1800764-Polymorphismus des ACE-Gens analysiert, für den die Alzgene-Datenbank hinsichtlich des Risikos einer AD eine Odds Ratio von 0,88 für das C-Allel angibt (Bertram et al. 2007). Dem C-Allel käme also demnach eine protektive Funktion zu. Untersuchungen über eine Assoziation zwischen ACE und der Progression der AD liegen bislang nicht vor. In der aktuellen Studie konnte gezeigt werden, dass C-AllelTrägerinnen eine schnellere MMST-Progression als Frauen ohne C-Allel aufwiesen. Dieses Ergebnis war zwar nicht statistisch signifikant $(p=0,083)$, kann aber als schwacher Trend interpretiert werden. Die Allelverteilung bei den männlichen Studienteilnehmern war nicht mit einem Unterschied in der Progressionsgeschwindigkeit assoziiert.

Zusammenfassend geben die Ergebnisse also Anlass zur Vermutung, dass das C-Allel bei weiblichen Individuen möglicherweise mit einer erhöhten Krankheitsprogression assoziiert sein könnte.

\subsubsection{LDLR}

Das Gen LDLR (low density lipid receptor) kodiert ein gleichnamiges Glykoprotein an der Zelloberfläche, welches eine wichtige Rolle in der Entfernung von Cholesterin und LDLPartikeln aus der Zirkulation spielt (Brown und Goldstein 1986; Herz und Bock 2002). Eine Mediatorfunktion übernimmt dabei das Apolipoprotein E, welches zudem in den A $\beta$ Metabolismus involviert ist (Kim et al. 2009). Welche Rolle der LDL-Rezetor in der Pathogenese der AD genau spielt, ist noch unklar. An Mausmodellen konnte gezeigt werden, 
dass ein Ausfall des LDL-Rezeptors beziehungsweise eine erhöhte Cholesterinlast mit einer erhöhten Amyloidablagerung assoziiert ist (Refolo et al. 2000; Cao et al. 2006). Zudem ließ sich in einer Studie zeigen, dass Cholesterin imstande ist, die proteolytische Aktivität der $\beta$ Sektretase zu stimulieren (Kalvodova et al. 2005).

Für den in der aktuellen Arbeit untersuchten LDLR-Polymorphismus (rs5930) konnte in der Alzgene-Datenbank eine Odds Ratio von 0,85 ermittelt werden (A vs. G) (Bertram et al. 2007). Eine Assoziation eines LDLR-Polymorphismus mit der Progressionsgeschwindigkeit der AD wurde in der Literatur bislang nicht beschrieben. In der vorliegenden Studie wiesen Frauen, die in dem untersuchten Polymorphismus ein G-Allel besaßen, eine höhere MMSTProgression als Nicht-G-Trägerinnen auf. Dieser Unterschied war statistisch signifikant $(p=$ 0,040). Bei den männlichen Individuen konnte kein allelabhängiger Unterschied in der Progressionsgeschwindigkeit festgestellt werden, sodass hier möglicherweise eine Wechselwirkung zwischen dem weiblichen Geschlecht und dem LDLR-Polymorphismus vorliegt.

\subsubsection{CST3}

CST3 kodiert das Protein Cystatin C, einen Inhibitor von Cystein-Proteasen (Yaffe et al. 2008). Es konnte gezeigt werden, dass Cystatin $C$ an $\beta$-Amyloid binden kann und dadurch die A $\beta$-Fibrillenbildung in-vitro vermindern kann (Sastre et al. 2004). Zudem wird eine Assoziation zwischen der Cystatin-C-Serumkonzentration und dem kognitiven Status von AD-Patienten vermutet (Chuo et al. 2007). Eine Metaanalyse der Alzgene-Datenbank ergab hinsichtlich des Risikos einer AD eine Odds Ratio von 1,13 für das A-Allel des rs1064039Polymorphismus von CST3 (Bertram et al. 2007). Eine Assoziation zwischen einem CST3Polymorphismus und der Verlaufsgeschwindigkeit der AD wurde bislang nicht beschrieben. In der aktuellen Arbeit zeigten Studienteilnehmer mit EOAD, die ein T-Allel im untersuchten CST3-Polymorphismus aufwiesen, eine deutlich geringere MMST-Progression als Nicht-TTräger mit EOAD (Anmerkung: Das T-Allel entspricht als Komplementärbase dem o.g. AAllel aus der Alzgene-Datenbank, da bei der in dieser Arbeit durchgeführten Genotypisierung der komplementäre DNS-Strang verwendet wurde). Dieser Unterschied war nah an der Grenze zur statistischen Signifikanz ( $p=0,053)$. Das Ergebnis lässt somit die Vermutung zu, dass das T-Allel möglicherweise einen verlangsamenden Effekt auf die Krankheitsprogression der EOAD hat. 


\subsubsection{ApoE, CLU, IL8 und EXOC3L2}

Für die Polymorphismen der Gene ApoE, CLU, IL8 und EXOC3L2 konnte in der vorliegenden Arbeit keine deutliche Assoziation mit der Progressionsgeschwindigkeit der untersuchten AD-Patienten gefunden werden. Während für den untersuchten $C L U$ Polymorphismus $\mathrm{Hu}$ et al. eine Assoziation mit der Progressionsgeschwindigkeit der AD zeigen konnten ( $\mathrm{Hu} \mathrm{X}$ et al. 2011), liegen für IL8 und EXOC3L2 in der Literatur bislang keine Daten hinsichtlich eines Einflusses auf die Progression vor.

Mit der möglichen Auswirkung des als Prädispositionsfaktor bekannten ApoEPolymorphismus (siehe Kapitel 1.3.3) auf die Progression haben sich dahingegen mehrere Forschergruppen beschäftigt. Eine ausführliche Übersicht der relevanten ApoE-Studien ist auf den nachfolgenden Seiten dargestellt (Tabellen 19-21). Insgesamt konnten dabei unterschiedliche Beobachtungen gemacht werden: Einige Autoren beschrieben einen schnelleren Verlauf bei Anwesenheit eines ع4-Allels (Tabelle 19), andere beobachteten diesen bei Abwesenheit (Tabelle 20). In der Mehrheit der Studien wurde jedoch eine fehlende Assoziation beschrieben (Tabelle 21). Auch in der vorliegenden Arbeit konnte kein signifikanter Unterschied zwischen den ApoE-Genotypen festgestellt werden, jedoch wies der $\varepsilon 4 / \varepsilon 4-G e n o t y p$ interessanterweise die geringste Progressionsgeschwindigkeit unter allen Genotypen auf. Diese Beobachtung ist möglicherweise ein diskreter Hinweis darauf, dass die Abwesenheit des $\varepsilon 4$-Allels mit einem schnelleren Verlauf assoziiert sein könnte. 
Tabelle 19: Übersicht relevanter ApoE-Studien Teil 1: Schnellere Krankheitsprogression der AD bei Anwesenheit von ApoE $\varepsilon 4$ (modifiziert und erweitert nach Kleiman et al. 2006, S. 78)

\begin{tabular}{|c|c|c|c|c|}
\hline Autor & Studienbeschreibung & $\begin{array}{l}\text { Diagnosekriterien / } \\
\text { Messung der Progression }\end{array}$ & $\begin{array}{l}\text { Follow-up } \\
\text { (Durchschnitt } \\
\text { in Jahren) }\end{array}$ & Details / Anmerkungen \\
\hline $\begin{array}{l}\text { (Cosentino et al. } \\
\text { 2008) }\end{array}$ & $\begin{array}{l}\text { Kohorte A (populationsbasiert) } \\
\text { Kohorte B (klinikbasiert); } \\
\text { Bildung von } 3 \text { Fallgruppen: } \\
\text { 1. } 199 \text { Fälle (inzident, aus A) } \\
\text { 2. } 215 \text { Fälle (prävalent, aus A) } \\
\text { 3. } 156 \text { Fälle (prävalent, aus B) }\end{array}$ & $\begin{array}{l}\text { DSM-III-R und } \\
\text { NINCDS-ADRDA; } \\
\text { Kohorte A: z-Score aus } \\
\text { kognitiver Testbatterie (Stern } \\
\text { et al. 1992) } \\
\text { Kohorte B: MMST }\end{array}$ & 4 & $\begin{array}{l}\text { Gruppe } 1 \text { (inzident): Schnellere } \\
\text { Progression bei Anwesenheit mindestens } \\
\text { eines } \varepsilon 4-A l l e l s ; \text { Gruppe } 2+3 \text { (prävalent): } \\
\text { Parallele Ergebnisse nach Anpassung an } \\
\text { Krankheitsschwere / Ausschluss } \\
\text { schwerstbetroffener Fälle }\end{array}$ \\
\hline $\begin{array}{l}\text { (Martins et al. } \\
\text { 2005) }\end{array}$ & $\begin{array}{l}218 \text { Fälle, Test in non-linearem } \\
\text { und linearem gemischtem } \\
\text { Modell }\end{array}$ & $\begin{array}{l}\text { NINCDS-ADRDA oder } \\
\text { Neuropathologische Kriterien } \\
\text { nach CERAD; } \\
\text { CAMCOG }\end{array}$ & 2,7 & $\begin{array}{l}\text { Nicht-lineares Modell: Schnellere } \\
\text { Progression mit steigender Anzahl der } \varepsilon 4- \\
\text { Allele, langsamere Progression bei } \\
\text { Anwesenheit eines } \varepsilon 2-\text { Allels }\end{array}$ \\
\hline $\begin{array}{l}\text { (Marra et al. } \\
\text { 2004) }\end{array}$ & $\begin{array}{l}30 \text { EOAD-Fälle (< } 66 \text { Jahre }) \\
41 \text { LOAD-Fälle (> } 69 \text { Jahre) }\end{array}$ & $\begin{array}{l}\text { NINCDS-ADRDA; } \\
\text { MMST, Testbatterie (Mental } \\
\text { Deterioration Battery) und } 4 \\
\text { weitere neuropsychologische } \\
\text { Tests }\end{array}$ & $\begin{array}{l}1,7 \\
\text { retrospektiv }^{\#}\end{array}$ & $\begin{array}{l}\text { Schnellere Progression bei } \varepsilon 4 \text {-Trägern (nur } \\
\text { EOAD) }\end{array}$ \\
\hline $\begin{array}{l}\text { (Hirono et al. } \\
\text { 2003) }\end{array}$ & 64 Fälle & $\begin{array}{l}\text { NINCDS-ADRDA; } \\
\text { ADAS-Cog }\end{array}$ & 1 & $\begin{array}{l}\text { Schnellere Progression mit steigender } \\
\text { Anzahl der } \varepsilon 4 \text {-Allele }\end{array}$ \\
\hline $\begin{array}{l}\text { (Kanai et al. } \\
\text { 1999) }\end{array}$ & 33 Fälle & $\begin{array}{l}\text { NINCDS-ADRDA; } \\
\text { MMST }\end{array}$ & 1,7 & Schnellere Progression bei $\varepsilon 4$-Trägern \\
\hline (Craft et al. 1998) & 201 Fälle & $\begin{array}{l}\text { NINCDS-ADRDA und } \\
\text { DSM-III-R; } \\
\text { DRS }\end{array}$ & 2,5 & $\begin{array}{l}\text { Schnellere Progression bei homozygoten } \\
\varepsilon 4 \text {-Trägern, langsamere Progression bei } \\
\varepsilon 2 \text {-Trägern }\end{array}$ \\
\hline
\end{tabular}

\footnotetext{
${ }^{\#}$ Erkrankungsbeginn wurde anhand anamnestischer Hinweise geschätzt, neuropsychologische Testung bei Erstdiagnose
} 
Tabelle 20: Übersicht relevanter ApoE-Studien Teil 2: Langsamere Krankheitsprogression der AD bei Anwesenheit von ApoE $\varepsilon 4$ (modifiziert und erweitert nach Kleiman et al. 2006, S. 78)

\begin{tabular}{|c|c|c|c|c|}
\hline Autor & Studienbeschreibung & $\begin{array}{l}\text { Diagnosekriterien / } \\
\text { Messung der Progression }\end{array}$ & $\begin{array}{l}\text { Follow-up } \\
\text { (Durchschnitt } \\
\text { in Jahren) }\end{array}$ & Details / Anmerkungen \\
\hline $\begin{array}{l}\text { (Schmidt et al. } \\
\text { 2010) }\end{array}$ & $\begin{array}{l}16 \text { Fälle mit rapid-progressiver } \\
\text { AD (initial CJK-Verdachtsfälle) }\end{array}$ & Post-mortem-Diagnose & $\begin{array}{l}2,2^{*} \\
\text { (retrospektiv) }\end{array}$ & Schnelle Progression bei Fehlen von $\varepsilon 4$ \\
\hline $\begin{array}{l}\text { (van der Vlies et } \\
\text { al. 2009) }\end{array}$ & $\begin{array}{l}99 \text { EOAD-Fälle, } 192 \text { LOAD- } \\
\text { Fälle }\end{array}$ & $\begin{array}{l}\text { NINCDS-ADRDA; } \\
\text { MMST }\end{array}$ & $\begin{array}{l}2,5(\mathrm{EOAD}) \\
2,3(\mathrm{LOAD})\end{array}$ & $\begin{array}{l}\text { Schnelle Progression bei negativem } \varepsilon 4 \text { (nur } \\
\text { bei EOAD) }\end{array}$ \\
\hline (Hoyt et al. 2005) & 151 Fälle & $\begin{array}{l}\text { NINCDS-ADRDA; } \\
\text { MMST, ADAS-Cog und } \\
\text { weitere Tests für spezifische } \\
\text { kognitive Funktionen, Tests } \\
\text { von funktionellen } \\
\text { Fähigkeiten (I-ADL, PSMS) }\end{array}$ & 3,3 & $\begin{array}{l}\text { Langsamere Progression bei homozygoten } \\
\varepsilon 4-\text { Trägern (nur in globalen kognitiven } \\
\text { Tests + in funktionellen Tests) }\end{array}$ \\
\hline (Stern et al. 1997) & 99 Fälle & $\begin{array}{l}\text { NINCDS-ADRDA; } \\
\text { Modifizierter MMST }\end{array}$ & 3,6 & Schnelle Progression bei Fehlen von $\varepsilon 4$ \\
\hline $\begin{array}{l}\text { (Frisoni et al. } \\
\text { 1995) }\end{array}$ & $\begin{array}{l}62 \text { LOAD-Fälle retrospektiv }{ }^{+} \\
\text {(davon } 28 \text { Fälle prospektiv) }\end{array}$ & $\begin{array}{l}\text { NINCDS-ADRDA; } \\
\text { MMST und CDR }\end{array}$ & $\begin{array}{l}3,9^{+} \\
\text {(retrospektiv) } \\
1,1 \\
\text { (prospektiv) }\end{array}$ & $\begin{array}{l}\text { Langsamere Progression bei } \varepsilon 4 \text {-Trägern } \\
\text { (nur LOAD) }\end{array}$ \\
\hline
\end{tabular}

\footnotetext{
"Mediane Überlebensrate (retrospektiv)

${ }^{+}$(geschätzter MMST-Score bei Erkrankungsbeginn - MMST Score bei Untersuchung)/ Krankheitsdauer
} 
Tabelle 21: Übersicht relevanter ApoE-Studien Teil 3: Kein Einfluss von ApoE $\varepsilon 4$ auf die Krankheitsprogression der AD (modifiziert und erweitert nach Kleiman et al. 2006, S. 78)

\begin{tabular}{|c|c|c|c|}
\hline Autor & Studienbeschreibung & $\begin{array}{l}\text { Diagnosekriterien / } \\
\text { Messung der Progression }\end{array}$ & $\begin{array}{l}\text { Follow-up Details / Anmerkungen } \\
\text { (Durchschnitt } \\
\text { in Jahren) }\end{array}$ \\
\hline $\begin{array}{l}\text { (Tschanz et al. } \\
\text { 2011) }\end{array}$ & 328 Fälle, populationsbasiert & $\begin{array}{l}\text { NINCDS-ADRDA; } \\
\text { MMST, CDR, NPI }\end{array}$ & 3,8 \\
\hline $\begin{array}{l}\text { (Kester et al. } \\
\text { 2009) }\end{array}$ & 130 Fälle & $\begin{array}{l}\text { NINCDS-ADRDA; } \\
\text { MMST }\end{array}$ & 2 \\
\hline $\begin{array}{l}\text { (Bracco et al. } \\
2007)\end{array}$ & 85 Fälle & $\begin{array}{l}\text { Eigenes Diagnostikprotokoll } \\
\text { (ähnlich NINCDS-ADRDA); } \\
\text { MMST, Short Story total } \\
\text { score }\end{array}$ & 3,3 \\
\hline $\begin{array}{l}\text { (Kleiman et al. } \\
\text { 2006) }\end{array}$ & 366 Fälle & $\begin{array}{l}\text { NINCDS-ADRDA oder } \\
\text { Neuropathologische Kriterien } \\
\text { nach CERAD; } \\
\text { MMST, ADAS-Cog, IADL, } \\
\text { ADCS-ADL }\end{array}$ & $\begin{array}{l}1,6 \\
\text { (retro- und } \\
\text { prospektiv) }\end{array}$ \\
\hline (Mori et al. 2002) & 55 Fälle & $\begin{array}{l}\text { NINCDS-ADRDA; } \\
\text { MMST, ADAS-Cog }\end{array}$ & 1 \\
\hline $\begin{array}{l}\text { (Aerssens et al. } \\
2001)\end{array}$ & 504 Fälle & $\begin{array}{l}\text { NINCDS-ADRDA; } \\
\text { ADAS-Cog, DAD }\end{array}$ & $\leq 1$ \\
\hline $\begin{array}{l}\text { (Farlow et al. } \\
\text { 1999) }\end{array}$ & 374 Fälle & $\begin{array}{l}\text { DSM-IV oder NINCDS- } \\
\text { ADRDA; } \\
\text { ADAS-COG, CIBIC-Plus }\end{array}$ & 0,4 \\
\hline $\begin{array}{l}\text { (Slooter et al. } \\
\text { 1999) }\end{array}$ & 85 Fälle & $\begin{array}{l}\text { NINCDS-ADRDA; } \\
\text { MMST }\end{array}$ & 4,4 \\
\hline
\end{tabular}


Tabelle 21 (Fortsetzung):

\begin{tabular}{|c|c|c|c|c|}
\hline Autor & Studienbeschreibung & $\begin{array}{ll}\text { Diagnosekriterien } \\
\text { Messung der Progression }\end{array}$ & $\begin{array}{l}\text { Follow-up } \\
\text { (Durchschnitt } \\
\text { in Jahren) }\end{array}$ & Details / Anmerkungen \\
\hline $\begin{array}{l}\text { (Lehtovirta et al. } \\
\text { 1998) }\end{array}$ & 31 Fälle & $\begin{array}{l}\text { NINCDS-ADRDA; } \\
\text { MMST, BCRS, Webster } \\
\text { scale, Hamilton scale, } \\
\text { neuropsychologische Tests }\end{array}$ & 3 & \\
\hline $\begin{array}{l}\text { (Jonker et al. } \\
\text { 1998) }\end{array}$ & $\begin{array}{l}3 \text { Untergruppen: AD } \quad(\mathrm{n}=34), \\
\text { „minimale Demenz“، } \\
\text { normale kognitive } \\
\begin{array}{l}(\mathrm{n}=54), \\
(\mathrm{n}=317)\end{array}\end{array}$ & $\begin{array}{l}\text { CAMDEX, NINCDS- } \\
\text { ADRDA und DSM-III-R; } \\
\text { CAMCOG }\end{array}$ & 3 & $\begin{array}{l}\text { Schnellere Progression nur bei } \varepsilon 4 \text {-Trägern } \\
\text { in präklinischer Phase, kein Einfluss bei } \\
\varepsilon 4 \text {-Trägern mit manifester Demenz }\end{array}$ \\
\hline $\begin{array}{l}\text { (Murphy et al. } \\
\text { 1997) }\end{array}$ & 86 Fälle & $\begin{array}{l}\text { NINCDS-ADRDA; } \\
\text { MMST }\end{array}$ & 3,6 & \\
\hline $\begin{array}{l}\text { (Asada et al. } \\
\text { 1996) }\end{array}$ & 70 Fälle & $\begin{array}{l}\text { NINCDS-ADRDA; } \\
\text { HDSR, CDR }\end{array}$ & 2,2 & $\begin{array}{l}\text { Kein Einfluss von } \varepsilon 4 \text { auf Progression, aber } \\
\text { schnellere Progression bei } \varepsilon 2\end{array}$ \\
\hline $\begin{array}{l}\text { (Dal Forno et al. } \\
\text { 1996) }\end{array}$ & 78 Fälle & $\begin{array}{l}\text { NINCDS-ADRDA oder } \\
\text { Neuropathologische Kriterien } \\
\text { nach CERAD; } \\
\text { MMST, neuropsychologische } \\
\text { Testbatterie, PGDRS }\end{array}$ & $\begin{array}{l}3,5 \\
(\text { PGDRS 5,5) }\end{array}$ & \\
\hline $\begin{array}{l}\text { (Holmes et al. } \\
\text { 1996) }\end{array}$ & $\begin{array}{l}145 \text { Fälle (LOAD, definiert als > } \\
60 \text { Jahre) }\end{array}$ & $\begin{array}{l}\text { NINCDS-ADRDA; } \\
\text { MMST, BDRS }\end{array}$ & 1,3 & \\
\hline (Kurz et al. 1996) & 51 Fälle & $\begin{array}{l}\text { ICD-10 Forschungskriterien; } \\
\text { CAMCOG, MMST, DS- } \\
\text { CAMDEX }\end{array}$ & 3 & \\
\hline
\end{tabular}


Tabelle 21 (Fortsetzung):

\begin{tabular}{|c|c|c|c|c|}
\hline Autor & Studienbeschreibung & $\begin{array}{l}\text { Diagnosekriterien } \\
\text { Messung der Progression }\end{array}$ & $\begin{array}{l}\text { Follow-up } \\
\text { (Durchschnitt } \\
\text { in Jahren) }\end{array}$ & Details / Anmerkungen \\
\hline $\begin{array}{l}\text { (Gomez-Isla et al. } \\
\text { 1996) }\end{array}$ & 153 Fälle & $\begin{array}{l}\text { NINCDS-ADRDA oder } \\
\text { neuropathologische Kriterien } \\
\text { nach Khachaturian; } \\
\text { IMC-BDS, ADL }\end{array}$ & 2,7 & \\
\hline $\begin{array}{l}\text { (Growdon et al. } \\
\text { 1996) }\end{array}$ & 66 Fälle & $\begin{array}{l}\text { NINCDS-ADRDA; } \\
\text { Testbatterie }\end{array}$ & 2 & \\
\hline $\begin{array}{l}\text { (Basun } \\
1995)\end{array}$ et al. & 60 Fälle (LOAD) & $\begin{array}{l}\text { DSM-III-R; } \\
\text { MMST }\end{array}$ & 3 & \\
\hline
\end{tabular}




\subsection{Schlussfolgerung}

Für den untersuchten rs541458-Polymorphismus des PICALM-Gens konnte bei C-AllelTrägern eine signifikant höhere durchschnittliche MMST-Progression als bei Nicht-C-Trägern nachgewiesen werden $(p=0,039)$. Auch für die Polymorphismen des BIN1- und TNK1-Gens ließen sich diskrete Hinweise auf eine mögliche Assoziation mit einem veränderten Krankheitsverlauf finden.

Nach Stratifikation der Daten ließ sich das Ergebnis des PICALM-Polymorphismus trendweise nur für Individuen mit LOAD nachweisen $(p=0,055)$. Zudem konnte für den untersuchten Polymorphismus des LDLR-Gens eine erhöhte MMST-Progression für weibliche G-Allel-Träger gezeigt werden $(p=0,040)$. Auch beim ACE-Gen deuteten sich geschlechtsabhängig diskrete Hinweise auf eine Assoziation mit einer modifizierten Progressionsgeschwindigkeit an, beim CST3-Gen in Abhängigkeit vom Krankheitsbeginn. Aufgrund sehr kleiner Gruppen nach Stratifikation ist die Aussagekraft dieser Ergebnisse jedoch möglicherweise eingeschränkt.

Polymorphismen, die zu der rapid-progressiven Form der AD (> 5 Punkte Verlust im MMST/Jahr) prädisponieren, konnten in dieser Studie nicht gefunden werden. Jedoch war der AG-Genotyp des BIN1-Gens signifikant häufiger in der langsamen Gruppe $(\leq 5$ Punkte Verlust im MMST/Jahr) anzutreffen $(p=0,026)$. Eine Zusammenfassung und Bewertung der Ergebnisse dieser Arbeit ist Tabelle 22 zu entnehmen.

Unter Berücksichtigung der kleinen Stichprobenanzahl der Studie, des durchgängig fehlenden monotonen Trends hinsichtlich der MMST-Progression mit steigender Risikoallelzahl und der fehlenden Korrektur für das multiple Testen reichen die Daten der vorliegenden Studie nicht aus, um valide Aussagen über eine Assoziation der Polymorphismen mit einem veränderten Krankheitsverlauf treffen zu können. Um die Ausprägung dieser möglichen Geneffekte genauer zu bestimmen, werden weitaus größere Studien benötigt, die auch andere Faktoren wie Umwelteinflüsse, Medikation, Komorbiditäten, kognitive Reserven oder auch Interaktionen zwischen den Polymorphismen berücksichtigen. Die Möglichkeit, die gefundenen Polymorphismen in der Zukunft eventuell als prognostische Marker verwenden und somit das Risiko des Krankheitsverlaufes bestimmen zu können, hätte sowohl für Patienten und ihre Angehörigen als auch für die behandelnden Ärzte große Bedeutung. 
Tabelle 22: Zusammenfassung der untersuchten Polymorphismen

\begin{tabular}{|c|c|c|c|c|}
\hline Gen & Polymorphismus & Einfluss auf Krankheitsprogression & Prädiktionsfaktor für rpAD* & Beurteilung \\
\hline ApoE & $\varepsilon 2 / 3 / 4$ & $\begin{array}{l}\text { Kein deutlicher Unterschied im Allel-/ } \\
\text { Genotyp-Vergleich }\end{array}$ & $\begin{array}{l}\text { Kein deutlicher Unterschied } \\
\text { zwischen „slow“ und „rapid““ }\end{array}$ & $\begin{array}{l}\text { Kein Hinweis für Assoziation mit } \\
\text { Krankheitsprogression }\end{array}$ \\
\hline$C L U$ & rs 11136000 & $\begin{array}{l}\text { Kein deutlicher Unterschied im Allel-/ } \\
\text { Genotyp-Vergleich }\end{array}$ & $\begin{array}{l}\text { Kein deutlicher Unterschied } \\
\text { zwischen „slow“ und „rapid“ }\end{array}$ & $\begin{array}{l}\text { Kein Hinweis für Assoziation mit } \\
\text { Krankheitsprogression }\end{array}$ \\
\hline PICALM & rs541458 & $\begin{array}{l}\text { C-Träger vs. Nicht-C-Träger: höhere } \\
\text { MMST-Progression }(p=0,039) \text {. } \\
\text { Nach Stratifikation Effekt nur bei } \\
\text { LOAD deutlich, hier nicht statistisch } \\
\text { signifikant }(p=0,055) \text {. }\end{array}$ & $\begin{array}{l}\text { Kein deutlicher Unterschied } \\
\text { zwischen „slow“ und „rapid“ }\end{array}$ & $\begin{array}{l}\text { Das C-Allel ist möglicherweise mit } \\
\text { einer erhöhten Progressions- } \\
\text { geschwindigkeit assoziiert, bevorzugt } \\
\text { bei LOAD }\end{array}$ \\
\hline EXOC $3 L 2$ & rs597668 & $\begin{array}{l}\text { Kein deutlicher Unterschied im Allel-/ } \\
\text { Genotyp-Vergleich }\end{array}$ & $\begin{array}{l}\text { Kein deutlicher Unterschied } \\
\text { zwischen „slow“ und „rapid“ }\end{array}$ & $\begin{array}{l}\text { Kein Hinweis für Assoziation mit } \\
\text { Krankheitsprogression }\end{array}$ \\
\hline BIN1 & rs744373 & $\begin{array}{l}\text { Nicht-A-Träger (GG) vs. A-Träger: } \\
\text { höhere MMST-Progression, nicht } \\
\text { statistisch signifikant }(p=0,114) \text {. } \\
\text { Weibliche Nicht-A-Träger vs. } \\
\text { weibliche A-Träger: höhere MMST- } \\
\text { Progression. Nicht statistisch } \\
\text { signifikant }(p=0,056) \text {. }\end{array}$ & $\begin{array}{l}\text { Genotyp AG vs. Genotypen } \\
\text { AA/GG: signifikant häufiger } \\
\text { in der langsam verlaufenden } \\
\text { Gruppe }\left(, \text { slow }^{\circ}\right) \text { nachweisbar } \\
(p=0,026) \text {. }\end{array}$ & $\begin{array}{l}\text { Ergebnisse lassen keine eindeutige } \\
\text { Interpretation zu, weitere Studien mit } \\
\text { größeren Teilnehmerzahlen müssen } \\
\text { erfolgen, um einen eindeutigen Effekt } \\
\text { zu bestätigen. }\end{array}$ \\
\hline $\begin{array}{l}G W A \\
14 q 32.13\end{array}$ & rs11622883 & $\begin{array}{l}\text { Kein deutlicher Unterschied im Allel-/ } \\
\text { Genotyp-Vergleich }\end{array}$ & $\begin{array}{l}\text { Kein deutlicher Unterschied } \\
\text { zwischen ,slow“ und „rapid“" }\end{array}$ & $\begin{array}{l}\text { Kein Hinweis für Assoziation mit } \\
\text { Krankheitsprogression }\end{array}$ \\
\hline
\end{tabular}

\footnotetext{
* Progressionsgeschwindigkeit im MMST > 5 Punkte/Jahr
} 
Tabelle 22 (Fortsetzung):

\begin{tabular}{|c|c|c|c|c|}
\hline Gen & Polymorphismus & Einfluss auf Krankheitsprogression & Prädiktionsfaktor für rpAD* & Beurteilung \\
\hline TNK1 & rs 1554948 & $\begin{array}{l}\text { MMST-Progression Genotyp AA }< \\
\text { Genotyp TT }<\text { Genotyp AT. Keine } \\
\text { statistische Signifikanz }(p=0,086)\end{array}$ & $\begin{array}{l}\text { Kein deutlicher Unterschied } \\
\text { zwischen „slow“ und „rapid““ }\end{array}$ & $\begin{array}{l}\text { Der Genotyp AA ist möglicherweise } \\
\text { mit einer langsameren Krankheits- } \\
\text { progression assoziiert } \\
\text { statistische Signifikanz) }\end{array}$ \\
\hline IL8 & rs 4073 & $\begin{array}{l}\text { Kein deutlicher Unterschied im Allel-/ } \\
\text { Genotyp-Vergleich }\end{array}$ & $\begin{array}{l}\text { Kein deutlicher Unterschied } \\
\text { zwischen „slow“ und „rapid“ }\end{array}$ & $\begin{array}{l}\text { Kein Hinweis für Assoziation mit } \\
\text { Krankheitsprogression }\end{array}$ \\
\hline$L D L R$ & rs5930 & $\begin{array}{l}\text { Weibliche G-Träger vs. weibliche } \\
\text { Nicht-G-Träger: höhere MMST- } \\
\text { Progression }(p=0,040)\end{array}$ & $\begin{array}{l}\text { Kein deutlicher Unterschied } \\
\text { zwischen „slow“ und „rapid““ }\end{array}$ & $\begin{array}{l}\text { Das G-Allel ist bei weiblichen } \\
\text { Individuen möglicherweise mit einer } \\
\text { erhöhten } \\
\text { assoziiert }\end{array}$ \\
\hline CST3 & rs 1064039 & $\begin{array}{l}\text { T-Allel-Träger (EOAD) vs. Nicht-T- } \\
\text { Allel-Träger (EOAD): geringere } \\
\text { MMST-Progression. Keine statis- } \\
\text { tische Signifikanz }(p=0,053) \text {. }\end{array}$ & $\begin{array}{l}\text { Kein deutlicher Unterschied } \\
\text { zwischen „slow“ und „rapid“" }\end{array}$ & $\begin{array}{l}\text { Das T-Allel ist bei Individuen mit } \\
\text { EOAD möglicherweise mit einer } \\
\text { verringerten } \\
\text { assoziiert } \\
\text { Signifikankheitsprogression }\end{array}$ \\
\hline$A C E$ & rs 1800764 & $\begin{array}{l}\text { Weibliche C-Allel-Träger vs. } \\
\text { weibliche Nicht-C-Allel-Träger: } \\
\text { schnellere MMST-Progression. Keine } \\
\text { statistische Signifikanz }(p=0,083)\end{array}$ & $\begin{array}{l}\text { Kein deutlicher Unterschied } \\
\text { zwischen „slow“ und „rapid“ }\end{array}$ & $\begin{array}{lcr}\text { Das C-Allel } & \text { ist bei } & \text { weiblichen } \\
\text { Individuen } & \text { möglicherweise } & \text { mit einer } \\
\text { erhöhten } & \text { Krankheitsprogression } \\
\text { assoziiert } & \text { (keine } & \text { statistische } \\
\text { Signifikanz) } & & \end{array}$ \\
\hline
\end{tabular}

\footnotetext{
* Progressionsgeschwindigkeit im MMST > 5 Punkte/Jahr
} 


\section{Zusammenfassung}

Genetische Einflüsse stellen in der Ätiologie der Alzheimer-Demenz (AD) eine zentrale Rolle dar. In den letzten Jahren konnte eine Vielzahl neuer Kandidatengene entdeckt werden, die in sogenannten Genome-wide association studies (GWAS) eine signifikante Assoziation zur AD zeigten. Inwieweit diese neu entdeckten Polymorphismen auch die Progressionsgeschwindigkeit der AD beeinflussen, ist bislang jedoch nur unzureichend untersucht.

Das Ziel dieser Arbeit war es daher, die 11 Polymorphismen mit der stärksten Assoziation zur $\mathrm{AD}$ in einem Kollektiv von 42 AD-Patienten hinsichtlich des Einflusses auf die Progressionsgeschwindigkeit zu untersuchen. Dazu wurde bei den Studienteilnehmern der Punktverlust im Mini-Mental-Status-Test (MMST) innerhalb eines Jahres gemessen. Unter den Polymorphismen wurde zusätzlich nach möglichen prädiktiven genetischen Markern für die „rapid-progressive AD“ (MMST-Verlust > 5 Punkte/Jahr) gesucht.

Für den untersuchten rs541458-Polymorphismus des PICALM-Gens ließ sich bei C-AllelTrägern eine signifikant höhere durchschnittliche MMST-Progression als bei Nicht-C-Trägern nachweisen $(p=0,039)$. Beim rs5930-Polymorphismus des LDLR-Gens konnte zudem für weibliche G-Allel-Träger eine signifikant erhöhte MMST-Progression gezeigt werden ( $p=$ 0,040). Prädiktive Marker für die rapid-progressive AD konnten nicht nachgewiesen werden. Der AG-Genotyp des untersuchten BIN1-Polymorphismus (rs744373) war jedoch signifikant häufiger in der langsamen Gruppe ( $\leq 5$ Punkte Verlust im MMST/Jahr) anzutreffen ( $p=$ 0,026).

Aufgrund der kleinen Stichprobenanzahl der Studie, des durchgängig fehlenden monotonen Trends hinsichtlich der MMST-Progression mit steigender Risikoallelzahl und der fehlenden Korrektur für das multiple Testen ist davon auszugehen, dass die Daten der vorliegenden Studie nicht ausreichen, um valide Aussagen über eine Assoziation der Polymorphismen mit einem veränderten Krankheitsverlauf treffen zu können. Da bisher keine vergleichbaren Studien vorliegen, sind weitere Untersuchungen mit weitaus größeren Teilnehmerzahlen nötig, um die Ausprägung dieser möglichen Effekte genauer zu bestimmen. Die Möglichkeit, die gefundenen Polymorphismen als prognostische Marker zu verwenden und somit das Risiko des Krankheitsverlaufes zu bestimmen, hätte sowohl für Patienten und ihre Angehörigen als auch für die behandelnden Ärzte große Bedeutung. 


\section{Anhang}

\subsection{Revidierte NINCDS-ADRDA-Kriterien nach Dubois}

Tabelle 23: Revidierte NINCDS-ADRDA-Kriterien

(modifiziert nach Dubois et al. 2007, S. 738)

Wahrscheinliche AD: A plus ein oder mehr unterstützende Kriterien B, C, D oder E:

\section{Kernkriterien:}

A. Frühe und signifikante Störung des episodischen Gedächtnisses, die außerdem folgende Kriterien erfüllt:

1. Progressive Änderung der Gedächtnisfunktionen seit $>6$ Monaten (berichtet durch Patienten oder durch Angehörige)

2. Objektive Beweise der Störung des episodischen Gedächtnisses in Tests: Erinnerungsstörung, die sich nicht durch Wiederholung bessert (Verstehen der Items und des zu erinnernden Inhaltes vorausgesetzt)

3. Die episodische Gedächtnisstörung kann isoliert oder in Assoziation mit anderen kognitiven Veränderungen zu Beginn oder im Verlauf der AD auftreten

\section{Unterstützende Kriterien:}

B. Atrophie des medialen temporalen Lappens

-Volumenverlust der Hippocampi, des entorhinalen Cortex, der Amygdala im MRT: qualitative Einschätzung mittels visuellem Scoring oder quantitative Volumetrie dieser Regionen (Vergleich zu gut charakterisierter, altersnormierter Population)

C. Abnormale Liquormarker -Niedrige $\mathrm{A} \beta_{1-42}$-Konzentration, erhöhte Gesamt-Tau-Konzentration oder erhöhte Phospho-TauKonzentration oder Kombination dieser drei

D. Spezifische Muster im PET

-Bilateral temporo-parietal reduzierter Glukosemetabolismus

E. Gesicherte autosomal-dominante AD-Mutation in der näheren Familie

\section{Ausschlusskriterien:}

- Plötzlicher Beginn

- Frühes Auftreten von Gangstörung, Anfällen, Verhaltensänderung

- Frühe fokalneurologische Defizite wie Hemiparese, Sensibilitätsstörungen, Gesichtsfelddefekte

- Frühe extrapyramidale Zeichen

- Non-AD-Demenz

- Ausgeprägte Depression

- $\quad$ Ausgeprägte Herz-Kreislauf-Krankheit

- Toxisch- oder metabolisch-bedingte Abnormitäten, die weiterer Abklärung bedürfen

- $\quad$ MRT-Flair- oder T2-Signal-Abnormitäten im medialen temporalen Lappen, die auf infektiöse oder vaskuläre Insulte hinweisen

\section{Kriterien für sichere AD:}

- $\quad$ Klinische UND bioptische/autoptische Sicherung

- $\quad$ Klinische UND genetische Sicherung (Mutation auf Chr. 1, 14 oder 21) 


\title{
6.2. Aufklärungsbogen (Version für Angehörige und Betreuer)
}

\author{
Klinik und Poliklinik für Neurologie \\ Abteilung Neurologie (Prof. Dr. M. Bähr) \\ Abteilung Klinische Neurophysiologie (Prof. Dr. W. Paulus)
}

Studienprojekt: „Charakterisierung von Prädiktoren (Klinik, Bildgebung, Liquor- und Serumparameter) rapid progressiver Verläufe des Morbus Alzheimer"

\section{"Aufklärung des gesetzlichen Betreuers bzw. der Angehörigen}

Sehr geehrte Angehörige, sehr geehrte Betreuerin, sehr geehrter Betreuer,

der Ihnen hier vorliegende Aufklärungstext beschreibt den Ablauf einer beobachtenden, wissenschaftlichen Studie zur Alzheimer'schen Krankheit. Wir würden im Folgenden gern herzlich dazu einladen, dass Ihr(e) erkankte(r) Angehörige(r) bzw. die von Ihnen betreute Person freiwillig an dieser Studie teilnimmt und dadurch einen Beitrag leisten kann, diese Erkrankung besser verstehen zu lernen. Auch Ihre freiwillige Mithilfe wäre im Rahmen dieser Studie gewünscht und erforderlich. Daher möchten wir auch Sie herzlich einladen, uns bei diesem Projekt zu unterstützen:

Bei einer ärztlichen Untersuchung zur Abklärung einer dementiellen Entwicklung (Vergesslichkeit und andere Symptome) kam bei Ihrem Angehörigen//hrer Angehörigen bzw. bei der von Ihnen zu betreuenden Person der Verdacht auf das Vorliegen eines Morbus Alzheimer auf.

Die Demenz vom Alzheimer-Typ ist die häufigste aller Demenzen und verläuft meistens langsam. Es scheint unter allen Patienten aber auch sehr wenige Fälle zu geben, bei denen eine Alzheimerdemenz sehr schnell fortschreitet. Warum dies so ist und welche Faktoren die Geschwindigkeit beeinflussen ist bisher unklar.

Im Rahmen dieser Studie möchten wir deshalb Patienten, bei denen ein Morbus Alzheimer festgestellt wurde, weiterführend untersuchen. Das Ziel ist es, den Verlauf der Krankheit bei diesen Patienten zu beobachten und Parameter zu finden, die möglicherweise später eine Vorhersage des Verlaufes der Krankheit erlauben, um eine bestmögliche Diagnose, Patientenaufklärung und Therapie gestalten zu können und um eine Grundlage zur weiteren Forschung auf diesem Gebiet zu schaffen. Im Rahmen dieser Studie wird neben einer körperlichen Untersuchung bei Ihrem/lhrer Angehörigen eine genaue Krankheitsgeschichte (Anamnese) erhoben. Zudem erfolgt eine umfangreiche neuropsychologische Testung. Dabei bitten wir zum Teil auch Angehörige/Betreuer (z.B. Kinder, Eltern oder Ehepartner) und/oder den Hausarzt alle drei Monate telefonisch um Mithilfe, sofern Sie uns Ihr Einverständnis hierzu geben und uns mitteilen, welche Bezugspersonen wir befragen dürfen. Eine Blutabnahme* ist notwendig, um entsprechende Laboruntersuchungen durchführen zu können. Einmal im Jahr, für einen Zeitraum von voraussichtlich drei Jahren, möchten wir gern den Patienten besuchen und die Untersuchungen und eine Blutabnahme wiederholen. 
Beim Vorliegen von Gedächtnisstörungen und anderen Hinweisen für einen über die Altersnorm hinausgehenden Gedächtnisschwund wird zur Abklärung aus diagnostischen Gründen eine Nervenwasserpunktion notwendig. Falls der vor Ort behandelnde Arzt bzw. Ärztin diese Prozedur durchführen möchte, wird er/sie Sie über Risiken und Komplikationen umfassend aufklären Wir würden gerne einige Milliliter dieser Nenvenflüssigkeit, die nach Abschluss aller Untersuchungen in der Regel zurückbleibt und ansonsten verworfen wird, nutzen, um neue diagnostische Parameter zu erarbeiten und bitten dafür um Ihre Zustimmung. Es findet keine zusätzliche Lumbalpunktion eigens im Rahmen dieser Studie statt.

Sämtliche erhobenen Daten werden vertraulich behandelt und nur für diese wissenschaftliche Studie verwendet. Alle Daten werden pseudonymisiert und im Rahmen des Datenschutzes kodiert und gespeichert.

Natürlich können Sie das Einverständnis zur Teilnahme Ihres Angehörigen an der Studie jederzeit auch ohne Angaben von Gründen beenden. Selbstverständlich entstehen für Ihren Angehörigen bei Nichtteilnahme oder Widerruf der Teilnahme keine Nachteile für die weitere Behandlung.

Sollten Sie jetzt oder im Verlauf noch weitere Fragen haben, stehen wir Ihnen gern zur Verfügung.

Mit freundlichen Grüßen

Prof. Dr. Inga Zerr

(Leiterin der Forschungsgruppe Prionerkrankungen und atypische Demenzen)

(Ort)

(Datum)

(aufklärender Studienarzt/Studienärztin)

\footnotetext{
* Die Risiken einer fachgerecht durchgeführten venösen Blutentnahme (in der Regel am Arm oder an der Hand), wie sie vielfach z.B. im Krankenhaus durchgeführt wird, sind extrem gering. Dennoch müssen wir Sie darauf hinweisen, dass es z.B. zu Nachblutungen, Hämatomen (,blaue Flecke“) und Entzündungen sowie Schmerzen an der Einstichstelle kommen kann.
} 


\subsection{Einwilligungsbogen (Version für Angehörige und Betreuer)}

\section{Klinik und Poliklinik für Neurologie}

Abteilung Neurologie (Prof. Dr. M. Bähr)

Abteilung Klinische Neurophysiologie (Prof. Dr. W. Paulus)

Studienprojekt: „Charakterisierung von Prädiktoren (Klinik, Bildgebung, Liquor- und Serumparameter) rapid progressiver Verläufe des Morbus Alzheimer"

\section{Einwilligungserklärung für den Betreuer/Angehörige}

Name des Patienten:

Geburtsdatum des Patienten:

Adresse des Patienten:

Telefonnummer des Patienten:

Hiermit bestätige ich, dass ich über das geplante Vorgehen im Rahmen der oben genannten Studie ausführlich aufgeklärt wurde. Diese Einwilligung gilt in Verbindung mit dem Aufklärungsschreiben, dessen Erhalt und Erläuterung ich hiermit bestätige.

Ich erkläre mich einverstanden, dass Herr/Frau: an dieser Studie teilnimmt und bin mit den dafür notwendigen Blutentnahmen einverstanden. Ich bin einverstanden damit, dass die von mir benannten Angehörigen/Betreuer/betreuenden Ärzte* mit zur Datenerhebung herangezogen werden. Ich erkläre weiterhin mein Einverständnis dafür, dass meine persönlichen Daten und die des o.g. von mir betreuten Patienten unter Berücksichtigung des Datenschutzes hierfür verwendet, verarbeitet und gespeichert werden. Ich bin mit dem in der Aufklärung beschriebenen Vorgehen innerhalb der Studie einverstanden. Ebenso bin ich mit denen in der Aufklärung beschriebenen, jährlichen Blutentnahmen einverstanden. Ich bin auch einverstanden, dass die in der Aufklärung beschriebenen Proben für wissenschaftliche Zwecke verwendet und Biomarker (inklusive genetischer Parameter) bestimmt werden. 
Ich behalte mir vor, ohne Angabe von Gründen, die Teilnahme des von mir betreuten Patienten/Angehörigen an dieser Studie zu beenden. Dadurch entstehen keinerlei Nachteile bezüglich der zukünftigen Behandlung.

Sämtliche erhobenen Daten werden vertraulich behandelt und nur für diese wissenschaftliche Studie verwendet. Alle Daten werden pseudonymisiert und im Rahmen des Datenschutzes kodiert und gespeichert.

Folgend benannte Personen dürfen zur Erhebung von persönlichen Daten mit herangezogen werden:

(Name, Adresse, Telefonnummer, Faxnummer, Beziehung zum Patienten)

(Bitte kennzeichnen (*), welchen Personen Untersuchungs/Laborergebnisse mitgeteilt werden dürfen und sollen)

1.

2.

3.

4.

5.

Name des hier einwilligenden Betreuers/Angehörigen (Adresse, Telnr., Beziehung zum Patienten): 


\subsection{Standardisierter Fragebogen}

\section{Fremdanamnese}

Modifziert. Informant Questionnaire on Cognitive Decline in the Elderly (IQCODE)

Name (Patient):

Name (Angehöriger):

Beziehung zum Patienten:

Haben Sie den Patienten regelmäßig gesehen?:

Wenn ja, wie lange, wie oft?:

1.Wann hat nach Ihrer Beobachtung und Ihrem Gefühl die Erkrankung der Patientin/des Patienten begonnen? Wann haben Sie zum ersten Mal deutlich festgestellt, dass „etwas nicht stimmt"?

\section{Datum:}

2. (Optional) Wann ist Ihnen zum ersten Mal aufgefallen, dass folgende Fähigkeiten sich bei dem Patienten/der Patientin deutlich verschlechtert haben? Bzw. wann traten diese Störungen zum ersten Mal auf?

\begin{tabular}{|c|c|c|c|c|}
\hline & Fähigkeit & $\begin{array}{l}\text { Erstmals gestört } \\
\text { seit (Datum)? }\end{array}$ & $\begin{array}{l}\text { Ist nicht } \\
\text { schlechter } \\
\text { geworden }\end{array}$ & Weiß nicht \\
\hline 1) & $\begin{array}{ll}\ldots & \text { Gesichter von Angehörigen und } \\
\text { Freunden erkennen }\end{array}$ & & & \\
\hline 2) & $\begin{array}{l}\text {... Namen von Angehörigen und Freunden } \\
\text { erinnern }\end{array}$ & & & \\
\hline 3) & $\begin{array}{l}\text {... Informationen über Angehörige und } \\
\text { Freunde erinnern wie z. B. Berufe, } \\
\text { Geburtstage, Adressen }\end{array}$ & & & \\
\hline 4) & ... sich an kürzlich Geschehenes erinnern & & & \\
\hline 5) & $\begin{array}{ll}\ldots & \text { sich nach einigen Tagen an } \\
& \text { Unterhaltungen erinnern }\end{array}$ & & & \\
\hline 6) & $\begin{array}{l}\ldots \quad \text { eine Unterhaltung führen, ohne zu ver- } \\
\text { gessen, was er / sie gerade sagen wollte }\end{array}$ & & & \\
\hline 7) & 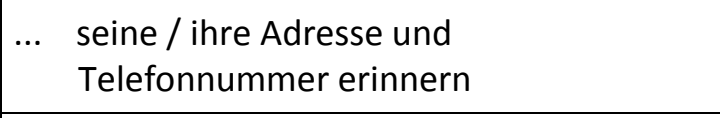 & & & \\
\hline 8) & $\begin{array}{l}\text {... Wochentag und Monat des heutigen } \\
\text { Tages erinnern }\end{array}$ & & & \\
\hline 9) & ... sich erinnern, wo Dinge hingehören & & & \\
\hline 10) & $\begin{array}{ll}\ldots & \begin{array}{l}\text { Dinge wiederfinden, die nicht an ihrem } \\
\text { gewohnten Platz abgelegt wurden }\end{array}\end{array}$ & & & \\
\hline 11) & ... sich an Veränderungen der Alltags- & & & \\
\hline
\end{tabular}




\begin{tabular}{|c|c|c|c|c|}
\hline & Routine gewöhnen & & & \\
\hline 12) & $\begin{array}{l}\text {... vertraute Haushalts- und Gartengeräte } \\
\text { benutzen }\end{array}$ & & & \\
\hline 13) & $\begin{array}{l}\ldots \text { den Umgang mit neuen Haushalts- oder } \\
\text { Gartengeräten erlernen }\end{array}$ & & & \\
\hline 14) & ... im Allgemeinen neue Dinge lernen & & & \\
\hline 15) & $\begin{array}{l}\text {... sich an Ereignisse aus seiner / ihrer } \\
\text { Jugendzeit erinnern }\end{array}$ & & & \\
\hline 16) & $\begin{array}{l}\ldots \text { sich an Dinge erinnern, die er / sie in der } \\
\text { Jugend gelernt hat }\end{array}$ & & & \\
\hline 17) & $\begin{array}{l}\text {... die Bedeutung unüblicher Worte } \\
\text { verstehen }\end{array}$ & & & \\
\hline 18) & $\begin{array}{l}\ldots \text { Zeitschriften- oder Zeitungsartikel } \\
\text { verstehen }\end{array}$ & & & \\
\hline 19) & $\begin{array}{l}\text {... eine Geschichte in einem Buch oder im } \\
\text { Fernsehen verfolgen }\end{array}$ & & & \\
\hline 20) & $\begin{array}{rr}\ldots & \text { einen Brief an Freunde oder für } \\
& \text { geschäftliche Zwecke verfassen }\end{array}$ & & & \\
\hline 21) & $\begin{array}{l}\text {... Wissen über wichtige historische } \\
\text { Ereignisse abrufen }\end{array}$ & & & \\
\hline 22) & ... Entscheidungen im Alltag treffen & & & \\
\hline 23) & ... beim Einkaufen mit Geld umgehen & & & \\
\hline 24) & $\begin{array}{ll}\ldots & \text { finanzielle Angelegenheiten regeln, z. B. } \\
& \text { Rente, Bankgeschäfte }\end{array}$ & & & \\
\hline 25) & $\begin{array}{l}\ldots \text { andere rechnerische Alltagsaufgaben } \\
\text { lösen, z. B. wissen, wie viele } \\
\text { Lebensmittel eingekauft werden } \\
\text { müssen, oder wie lange der letzte } \\
\text { Besuch von Angehörigen oder Freunden } \\
\text { her ist }\end{array}$ & & & \\
\hline 26) & $\begin{array}{l}\text {.. } \text { seine / ihre geistigen Fähigkeiten } \\
\text { nutzen, um zu verstehen, was passiert, } \\
\text { um Dinge zu beurteilen und } \\
\text { Schlussfolgerungen zu ziehen }\end{array}$ & & & \\
\hline
\end{tabular}




\section{Anamnese und Untersuchung}

Beginn der dementiellen Symptomatik (siehe Angehörigenbefragung (als Hilfsmittel dienen die modifizierten IQCODE Fragen) und Akten):

Datum:

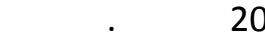

in Monaten vor Erstuntersuchung: Monate

Größe: $\mathrm{m}$

Gewicht Erstuntersuchung: Gewichtsverlust:

wieviel Innerhalb $\mathrm{kg}$

Bildungsgrad des Patienten:

\section{Erstuntersuchung (Symptome seit Krankheitsbeginn)}

Standardanamnesebogen ausgefüllt und abgeheftet.

Standarduntersuchungsbogen ausgefüllt und abgeheftet.

\begin{tabular}{|l|l|l|l|}
\hline & $\mathrm{Ja}$ & Nein & unklar \\
\hline Gedächtnisstörung & $\mathrm{Ja}$ & Nein & unklar \\
\hline Desorientiertheit/Verwirrtheit & $\mathrm{Ja}$ & Nein & unklar \\
\hline Konzentrationsstörung & $\mathrm{Ja}$ & Nein & unklar \\
\hline Wesensänderung & $\mathrm{Ja}$ & Nein & unklar \\
\hline Psychomot. Verlangsamung & $\mathrm{Ja}$ & Nein & unklar \\
\hline Wahnvorstellungen & $\mathrm{Ja}$ & Nein & unklar \\
\hline Halluzinationen, wenn ja, welche: & $\mathrm{Ja}$ & Nein & unklar \\
\hline Depression & & & \\
\hline Angst & $\mathrm{Ja}$ & Nein & unklar \\
\hline
\end{tabular}




\begin{tabular}{|c|c|c|c|}
\hline Agression & $\mathrm{Ja}$ & Nein & unklar \\
\hline Affektlabilität & $\mathrm{Ja}$ & Nein & unklar \\
\hline Apathie, Antriebsminderung & $\mathrm{Ja}$ & Nein & unklar \\
\hline Fresssucht & $\mathrm{Ja}$ & Nein & unklar \\
\hline Apptitlosigkeit & $\mathrm{Ja}$ & Nein & unklar \\
\hline Gewichtszunahme & $\mathrm{Ja}$ & Nein & unklar \\
\hline Gewichtsabnahme & $\mathrm{Ja}$ & Nein & unklar \\
\hline Einschlafstörung & $\mathrm{Ja}$ & Nein & unklar \\
\hline Durchschlafstörung & $\mathrm{Ja}$ & Nein & unklar \\
\hline Tag/Nachtumkehr & $\mathrm{Ja}$ & Nein & unklar \\
\hline Inkontinenz & $\mathrm{Ja}$ & Nein & unklar \\
\hline Enthemmung & $\mathrm{Ja}$ & Nein & unklar \\
\hline Witzelsucht & $\mathrm{Ja}$ & Nein & unklar \\
\hline Euphorie & $\mathrm{Ja}$ & Nein & unklar \\
\hline Perseveration & $\mathrm{Ja}$ & Nein & unklar \\
\hline Distanzlosigkeit & $\mathrm{Ja}$ & Nein & unklar \\
\hline Apraxie & $\mathrm{Ja}$ & Nein & unklar \\
\hline \multirow[t]{2}{*}{ Räumliche Orientierungsstörung } & $\mathrm{Ja}$ & Nein & unklar \\
\hline & $\mathrm{Ja}$ & Nein & unklar \\
\hline Sprach/Sprechstörung & $\mathrm{Ja}$ & Nein & unklar \\
\hline Motorische Aphasie & $\mathrm{Ja}$ & Nein & unklar \\
\hline Sensorische Aphasie & $\mathrm{Ja}$ & Nein & unklar \\
\hline Sensomotorische Aphasie & $\mathrm{Ja}$ & Nein & unklar \\
\hline Amnestische Aphasie & $\mathrm{Ja}$ & Nein & unklar \\
\hline Dysarthrie & $\mathrm{Ja}$ & Nein & unklar \\
\hline \multirow[t]{2}{*}{ Bulbäre/pseudobulbäre Sprache } & $\mathrm{Ja}$ & Nein & unklar \\
\hline & $\mathrm{Ja}$ & Nein & unklar \\
\hline Sehstörung & $\mathrm{Ja}$ & Nein & unklar \\
\hline Kortikal & $\mathrm{Ja}$ & Nein & unklar \\
\hline Nichtkortikal & $\mathrm{Ja}$ & Nein & unklar \\
\hline \multirow[t]{2}{*}{ Ophtalmologisch } & $\mathrm{Ja}$ & Nein & unklar \\
\hline & $\mathrm{Ja}$ & Nein & unklar \\
\hline Gangataxie & $\mathrm{Ja}$ & Nein & unklar \\
\hline Standataxie & $\mathrm{Ja}$ & Nein & unklar \\
\hline Rumpfataxie & $\mathrm{Ja}$ & Nein & unklar \\
\hline Extremitätenataxie & $\mathrm{Ja}$ & Nein & unklar \\
\hline Dysmetrie & $\mathrm{Ja}$ & Nein & unklar \\
\hline Dys/Bradydiadochokinese & $\mathrm{Ja}$ & Nein & unklar \\
\hline Rigor & $\mathrm{Ja}$ & Nein & unklar \\
\hline Zahnradphänomen & $\mathrm{Ja}$ & Nein & unklar \\
\hline Hypokinese & $\mathrm{Ja}$ & Nein & unklar \\
\hline $\begin{array}{l}\text { Hyperkinese } \\
\text { Welche: }\end{array}$ & $\mathrm{Ja}$ & Nein & unklar \\
\hline Ruhetremor & $\mathrm{Ja}$ & Nein & unklar \\
\hline Kleinschrittiger Gang & $\mathrm{Ja}$ & Nein & unklar \\
\hline Myoklonien & $\mathrm{Ja}$ & Nein & unklar \\
\hline Reflexdifferenzen & $\mathrm{Ja}$ & Nein & unklar \\
\hline \multirow[t]{2}{*}{ Pyramidenbahnzeichen } & $\mathrm{Ja}$ & Nein & unklar \\
\hline & $\mathrm{Ja}$ & Nein & unklar \\
\hline Sensibilitätsstörungen & $\mathrm{Ja}$ & Nein & unklar \\
\hline
\end{tabular}




\begin{tabular}{|l|l|l|l|}
\hline Dysästhesie & $\mathrm{Ja}$ & Nein & unklar \\
\hline Parästhesie & $\mathrm{Ja}$ & Nein & unklar \\
\hline Hypästhesie & $\mathrm{Ja}$ & Nein & unklar \\
\hline Hyperästhesie & $\mathrm{Ja}$ & Nein & unklar \\
\hline Hyperpathie & $\mathrm{Ja}$ & Nein & unklar \\
\hline Allodynie & $\mathrm{Ja}$ & Nein & unklar \\
\hline & $\mathrm{Ja}$ & Nein & unklar \\
\hline Epilleptische Anfälle & $\mathrm{Ja}$ & Nein & unklar \\
\hline Generalisierte Anfälle & $\mathrm{Ja}$ & Nein & unklar \\
\hline Fokale Anfälle & $\mathrm{Ja}$ & Nein & unklar \\
\hline & $\mathrm{Ja}$ & Nein & unklar \\
\hline Vegetative Symptome & $\mathrm{Ja}$ & Nein & unklar \\
\hline Hyperhidrosis & $\mathrm{Ja}$ & Nein & unklar \\
\hline Orthostatische Dysregulation & $\mathrm{Ja}$ & Nein & unklar \\
\hline Tachykardie & $\mathrm{Ja}$ & Nein & unklar \\
\hline Bradykardie & $\mathrm{Ja}$ & Nein & unklar \\
\hline Hustenreiz & $\mathrm{Ja}$ & Nein & unklar \\
\hline & $\mathrm{Ja}$ & Nein & unklar \\
\hline Primitivreflexe & $\mathrm{Ja}$ & Nein & unklar \\
\hline Dysphagie & $\mathrm{Ja}$ & Nein & unklar \\
\hline
\end{tabular}

\section{Vorerkrankungen}

Diabetes mell: Hypertonus:

Sonstige VE:

$\square$ ja $\quad \square$ nein

Nikotinabusus Packyears

Alkohol: $\square$ nein $\square$ selten $\square$ tägl. $\square$ mehrmals tägl: 
Aktuelle Medikation

Memantine

Cholinesterasehemmer

Blutruckmedikation

Antiaggregation (zb ASS)

Antikoagulation (zb Marcumar)

Benzodiazepine

Neuroleptika

Antidepressiva

Opiate

$\square$ nein

$\square$ nein

$\square$ nein

$\square$ nein

$\square$ nein

$\square$ nein

$\square$ nein

$\square$ nein

\section{Spezielle Familienanamnese}

Bitte ankreuzen

\begin{tabular}{|c|c|c|c|c|c|}
\hline $\begin{array}{l}\text { Verwandter } \\
\text { (Verwandschafts } \\
\text { grad) }\end{array}$ & $\begin{array}{l}\text { Demenz } \\
\text { Onset < } \\
65 \mathrm{LJ}\end{array}$ & $\begin{array}{l}\text { Demenz } \\
\text { Onset > } \\
65 \mathrm{LJ}\end{array}$ & $\begin{array}{l}\text { Keine } \\
\text { Demenz }\end{array}$ & Alzheimer? & Unklar \\
\hline & & & & & \\
\hline & & & & & \\
\hline & & & & & \\
\hline & & & & & \\
\hline & & & & & \\
\hline & & & & & \\
\hline & & & & & \\
\hline & & & & & \\
\hline & & & & & \\
\hline & & & & & \\
\hline & & & & & \\
\hline & & & & & \\
\hline & & & & & \\
\hline & & & & & \\
\hline
\end{tabular}

Andere Erkrankungen in der FA (wer (Verwandtschaftsgrad), was):

\section{Stammbaum (bei auffälliger Familienanamnese, sonst optional)}

Hier Stammbaum auf getrenntem Blatt einfügen. 


\section{Literaturverzeichnis}

Abellan van Kan G, Rolland Y, Nourhashemi F, Coley N, Andrieu S und Vellas B (2009):

Cardiovascular disease risk factors and progression of Alzheimer's disease. Dement Geriatr Cogn Disord 27(3): 240-6.

Aebi C: Validierung der neuropsychologischen Testbatterie CERAD-NP: eine Multi-Center Studie. Phil. Diss. Basel 2002.

Aerssens J, Raeymaekers P, Lilienfeld S, Geerts H, Konings F und Parys W (2001): APOE genotype: no influence on galantamine treatment efficacy nor on rate of decline in Alzheimer's disease. Dement Geriatr Cogn Disord 12(2): 69-77.

Alzheimer A (1907): Über eine eigenartige Erkrankung der Hirnrinde. Zeitschrift für Psychiatrie und psychiatrisch-gerichtliche Medizin 64: 146-148.

APA: Diagnostic and Statistical Manual of Mental Disorders, Fourth Edition, Text Revision; APA, Washington, DC 2000.

Asada T, Kariya T, Yamagata Z, Kinoshita T und Asaka A (1996): ApoE epsilon 4 allele and cognitive decline in patients with Alzheimer's disease. Neurology 47(2): 603.

Atchison TB, Massman PJ und Doody RS (2007): Baseline cognitive function predicts rate of decline in basic-care abilities of individuals with dementia of the Alzheimer's type. Arch Clin Neuropsychol 22(1): 99-107.

Baig S, Joseph SA, Tayler H, Abraham R, Owen MJ, Williams J, Kehoe PG und Love S (2010): Distribution and expression of picalm in Alzheimer disease. J Neuropathol Exp Neurol 69(10): 1071-7.

Barnes DE und Yaffe K (2011): The projected effect of risk factor reduction on Alzheimer's disease prevalence. Lancet Neurol 10(9): 819-28.

Bartus RT (2000): On neurodegenerative diseases, models, and treatment strategies: lessons learned and lessons forgotten a generation following the cholinergic hypothesis. Exp Neurol 163(2): 495-529. 
Bartus RT, Dean RL, 3rd, Beer B und Lippa AS (1982): The cholinergic hypothesis of geriatric memory dysfunction. Science 217(4558): 408-14.

Basun H, Grut M, Winblad B und Lannfelt L (1995): Apolipoprotein epsilon 4 allele and disease progression in patients with late-onset Alzheimer's disease. Neurosci Lett 183(1-2): 32-4.

Bekris LM, Yu CE, Bird TD und Tsuang DW (2010): Genetics of Alzheimer disease. J Geriatr Psychiatry Neurol 23(4): 213-27.

Belbin O, Dunn JL, Chappell S, Ritchie AE, Ling Y, Morgan L, Pritchard A, Warden DR, Lendon CL, Lehmann DJ, et al. (2008): A SNP in the ACT gene associated with astrocytosis and rapid cognitive decline in AD. Neurobiol Aging 29(8): 1167-76.

Belbin O, Beaumont H, Warden D, Smith AD, Kalsheker N und Morgan K (2009): PSEN1 polymorphisms alter the rate of cognitive decline in sporadic Alzheimer's disease patients. Neurobiol Aging 30(12): 1992-9.

Berti V, Osorio RS, Mosconi L, Li Y, De Santi S und de Leon MJ (2010): Early detection of Alzheimer's disease with PET imaging. Neurodegener Dis $\underline{7}(1-3):$ 131-5.

Bertram L: The genetics of Alzheimer's disease. In: Handbook of Clinical Neurology. Duyckaerts C und Litvan I (Hrsg.); Vol. 89; Elsevier, Amsterdam 2008, 223-32.

Bertram L und Tanzi RE (2008): Thirty years of Alzheimer's disease genetics: the implications of systematic meta-analyses. Nat Rev Neurosci $\underline{9}(10):$ 768-78.

Bertram L und Tanzi RE (2009): Genome-wide association studies in Alzheimer's disease. Hum Mol Genet 18(R2): R137-45.

Bertram L, McQueen MB, Mullin K, Blacker D und Tanzi RE (2007): Systematic meta-analyses of Alzheimer disease genetic association studies: the AlzGene database. Nat Genet 39(1): 17-23. (www.alzgene.org)

Bickel H: Stand der Epidemiologie. In: Weißbuch Demenz. Versorgungssituation relevanter Demenzerkrankungen in Deutschland. Hallauer J und Kurz A (Hrsg.); Georg Thieme Verlag, Stuttgart 2002, 10-14. 
Bickel H: Epidemiologie und Gesundheitsökonomie. In: Demenzen. Wallesch CW und Förstl H (Hrsg.); Georg Thieme Verlag, Stuttgart 2005, 1-15.

Bird TD (2008): Genetic aspects of Alzheimer disease. Genet Med 10(4): 231-9.

Blennow K (2004): Cerebrospinal fluid protein biomarkers for Alzheimer's disease. NeuroRx 1 (2): 213-25.

Blennow K und Hampel H (2003): CSF markers for incipient Alzheimer's disease. Lancet Neurol 2(10): 605-13.

Blennow K, de Leon MJ und Zetterberg H (2006): Alzheimer's disease. Lancet 368(9533): 387-403.

Bossu P, Ciaramella A, Moro ML, Bellincampi L, Bernardini S, Federici G, Trequattrini A, Macciardi F, Spoletini I, Di Iulio F, et al. (2007): Interleukin 18 gene polymorphisms predict risk and outcome of Alzheimer's disease. J Neurol Neurosurg Psychiatry 78 (8): 807-11.

Braak E, Griffing K, Arai K, Bohl J, Bratzke H und Braak H (1999): Neuropathology of Alzheimer's disease: what is new since A. Alzheimer? Eur Arch Psychiatry Clin Neurosci 249 Suppl 3: 1422 .

Bracco L, Piccini C, Baccini M, Bessi V, Biancucci F, Nacmias B, Bagnoli S und Sorbi S (2007): Pattern and progression of cognitive decline in Alzheimer's disease: role of premorbid intelligence and ApoE genotype. Dement Geriatr Cogn Disord 24(6): 483-91.

Brown MS und Goldstein JL (1986): A receptor-mediated pathway for cholesterol homeostasis. Science 232(4746): 34-47.

Buee L, Bussiere T, Buee-Scherrer V, Delacourte A und Hof PR (2000): Tau protein isoforms, phosphorylation and role in neurodegenerative disorders. Brain Res Brain Res Rev 33(1): 95130.

Burns A, Jacoby R und Levy R (1990): Psychiatric phenomena in Alzheimer's disease. IV: Disorders of behaviour. Br J Psychiatry 157: 86-94.

Burns A, Jacoby R und Levy R (1991): Neurological signs in Alzheimer's disease. Age Ageing 20(1): $45-51$. 
Campion D, Dumanchin C, Hannequin D, Dubois B, Belliard S, Puel M, Thomas-Anterion C, Michon A, Martin C, Charbonnier F, et al. (1999): Early-onset autosomal dominant Alzheimer disease: prevalence, genetic heterogeneity, and mutation spectrum. Am J Hum Genet 65(3): 664-70.

Cao D, Fukuchi K, Wan H, Kim H und Li L (2006): Lack of LDL receptor aggravates learning deficits and amyloid deposits in Alzheimer transgenic mice. Neurobiol Aging 27(11): 1632-43.

Carcaillon L, Peres K, Pere JJ, Helmer C, Orgogozo JM und Dartigues JF (2007): Fast cognitive decline at the time of dementia diagnosis: a major prognostic factor for survival in the community. Dement Geriatr Cogn Disord 23(6): 439-45.

Carrell RW und Lomas DA (2002): Alpha1-antitrypsin deficiency--a model for conformational diseases. N Engl J Med 346(1): 45-53.

Caselli RJ, Couce ME, Osborne D, Deen HG und Parisi JP (1998): From slowly progressive amnesic syndrome to rapidly progressive Alzheimer disease. Alzheimer Dis Assoc Disord 12(3): 2513.

Chai CK (2007): The genetics of Alzheimer's disease. Am J Alzheimers Dis Other Demen 22(1): 37-

41.

Chiappelli M, Nasi M, Cossarizza A, Porcellini E, Tumini E, Pinti M, Troiano L, Franceschi M und Licastro F (2006): Polymorphisms of fas gene: relationship with Alzheimer's disease and cognitive decline. Dement Geriatr Cogn Disord 22(4): 296-300.

Chuo LJ, Sheu WH, Pai MC und Kuo YM (2007): Genotype and plasma concentration of cystatin C in patients with late-onset Alzheimer disease. Dement Geriatr Cogn Disord 23(4): 251-7.

Corder EH, Saunders AM, Strittmatter WJ, Schmechel DE, Gaskell PC, Small GW, Roses AD, Haines JL und Pericak-Vance MA (1993): Gene dose of apolipoprotein E type 4 allele and the risk of Alzheimer's disease in late onset families. Science 261(5123): 921-3.

Corey-Bloom J (2002): The ABC of Alzheimer's disease: cognitive changes and their management in Alzheimer's disease and related dementias. Int Psychogeriatr 14 Suppl 1: 51-75. 
Cortes F, Gillette-Guyonnet S, Nourhashemi F, Andrieu S, Cantet C und Vellas B (2005): Recent data on the natural history of Alzheimer's disease: results from the REAL.FR Study. J Nutr Health Aging 9(2): 86-93.

Cortes F, Nourhashemi F, Guerin O, Cantet C, Gillette-Guyonnet S, Andrieu S, Ousset PJ und Vellas B (2008): Prognosis of Alzheimer's disease today: a two-year prospective study in 686 patients from the REAL-FR Study. Alzheimers Dement 4(1): 22-9.

Cosentino S, Scarmeas N, Helzner E, Glymour MM, Brandt J, Albert M, Blacker D und Stern Y (2008): APOE epsilon 4 allele predicts faster cognitive decline in mild Alzheimer disease. Neurology $\underline{70}$ (19 Pt 2): 1842-9.

Craft S, Teri L, Edland SD, Kukull WA, Schellenberg G, McCormick WC, Bowen JD und Larson EB (1998): Accelerated decline in apolipoprotein E-epsilon4 homozygotes with Alzheimer's disease. Neurology 51(1): 149-53.

Crews FT, Kurian P und Freund G (1994): Cholinergic and serotonergic stimulation of phosphoinositide hydrolysis is decreased in Alzheimer's disease. Life Sci 55(25-26): 19932002 .

Cruts M und Van Broeckhoven C (1998): Molecular genetics of Alzheimer's disease. Ann Med 30(6): 560-5.

Cruts M, Theuns J und Van Broeckhoven C (2012): Locus-specific mutation databases for neurodegenerative brain diseases. Hum Mutat 33(9): 1340-4.

Dal Forno G, Rasmusson DX, Brandt J, Carson KA, Brookmeyer R, Troncoso J und Kawas CH (1996): Apolipoprotein E genotype and rate of decline in probable Alzheimer's disease. Arch Neurol 53(4): 345-50.

Davies L, Wolska B, Hilbich C, Multhaup G, Martins R, Simms G, Beyreuther K und Masters CL (1988): A4 amyloid protein deposition and the diagnosis of Alzheimer's disease: prevalence in aged brains determined by immunocytochemistry compared with conventional neuropathologic techniques. Neurology $\underline{38}(11):$ 1688-93. 
Di Paolo G, Sankaranarayanan S, Wenk MR, Daniell L, Perucco E, Caldarone BJ, Flavell R, Picciotto MR, Ryan TA, Cremona O, et al. (2002): Decreased synaptic vesicle recycling efficiency and cognitive deficits in amphiphysin 1 knockout mice. Neuron 33(5): 789-804.

Doody RS, Massman P und Dunn JK (2001): A method for estimating progression rates in Alzheimer disease. Arch Neurol 58(3): 449-54.

Dubois B, Feldman HH, Jacova C, Dekosky ST, Barberger-Gateau P, Cummings J, Delacourte A, Galasko D, Gauthier S, Jicha G, et al. (2007): Research criteria for the diagnosis of Alzheimer's disease: revising the NINCDS-ADRDA criteria. Lancet Neurol 6(8): 734-46.

Dumont C, Voisin T, Nourhashemi F, Andrieu S, Koning M und Vellas B (2005): Predictive factors for rapid loss on the mini-mental state examination in Alzheimer's disease. J Nutr Health Aging 9 (3): 163-7.

Emmerling MR, Moore CJ, Doyle PD, Carroll RT und Davis RE (1993): Phospholipase A2 activation influences the processing and secretion of the amyloid precursor protein. Biochem Biophys Res Commun 197(1): 292-7.

Etgen T, Sander D, Bickel H und Forstl H (2011): Mild cognitive impairment and dementia: the importance of modifiable risk factors. Dtsch Ärztebl Int 108(44): 743-50.

Fahn S, Elton RL, Members of the UPDRS Development Committee: Unified Parkinson's Disease Rating Scale. In: Recent developments in Parkinson's Disease. Fahn S, Marsden CD, Goldstein M und Calne DB (Hrsg.); Vol. 2; MacMillan, New York 1987, 153-163.

Farlow MR, Cyrus PA, Nadel A, Lahiri DK, Brashear A und Gulanski B (1999): Metrifonate treatment of AD: influence of APOE genotype. Neurology 53(9): 2010-6.

Felschow DM, Civin CI und Hoehn GT (2000): Characterization of the tyrosine kinase Tnk1 and its binding with phospholipase C-gamma1. Biochem Biophys Res Commun 273(1): 294-301.

Ferri CP, Prince M, Brayne C, Brodaty H, Fratiglioni L, Ganguli M, Hall K, Hasegawa K, Hendrie H, Huang Y, et al. (2005): Global prevalence of dementia: a Delphi consensus study. Lancet 366(9503): 2112-7. 
Folstein MF, Folstein SE und McHugh PR (1975): "Mini-mental state". A practical method for grading the cognitive state of patients for the clinician. J Psychiatr Res 12(3): 189-98.

Förstl H und Kurz A (1999): Clinical features of Alzheimer's disease. Eur Arch Psychiatry Clin Neurosci 249(6): 288-90.

Förstl H, Burns A, Levy R, Cairns N, Luthert P und Lantos P (1992): Neurologic signs in Alzheimer's disease. Results of a prospective clinical and neuropathologic study. Arch Neurol 49(10): $1038-42$.

Frisoni GB, Govoni S, Geroldi C, Bianchetti A, Calabresi L, Franceschini G und Trabucchi M (1995): Gene dose of the epsilon 4 allele of apolipoprotein $\mathrm{E}$ and disease progression in sporadic lateonset Alzheimer's disease. Ann Neurol 37(5): 596-604.

Gauthier S, Reisberg B, Zaudig M, Petersen RC, Ritchie K, Broich K, Belleville S, Brodaty H, Bennett D, Chertkow H, et al. (2006): Mild cognitive impairment. Lancet 367(9518): 1262-70.

Goate A, Chartier-Harlin MC, Mullan M, Brown J, Crawford F, Fidani L, Giuffra L, Haynes A, Irving N, James L, et al. (1991): Segregation of a missense mutation in the amyloid precursor protein gene with familial Alzheimer's disease. Nature 349(6311): 704-6.

Goldberg RJ (2007): Alzheimer's disease. Compr Ther 33(2): 58-64.

Gomez-Isla T, West HL, Rebeck GW, Harr SD, Growdon JH, Locascio JJ, Perls TT, Lipsitz LA und Hyman BT (1996): Clinical and pathological correlates of apolipoprotein E epsilon 4 in Alzheimer's disease. Ann Neurol 39(1): 62-70.

Gomez-Isla T, Spires T, De Calignon A und Hyman BT: Neuropathology of Alzheimer's disease. In: Handbook of Clinical Neurology. Duyckaerts C und Litvan I (Hrsg.); Vol. 89; Elsevier, Amsterdam 2008, 233-43.

Greenamyre JT und Young AB (1989): Excitatory amino acids and Alzheimer's disease. Neurobiol Aging 10(5): 593-602.

Greenberg SM, Rebeck GW, Vonsattel JP, Gomez-Isla T und Hyman BT (1995): Apolipoprotein E epsilon 4 and cerebral hemorrhage associated with amyloid angiopathy. Ann Neurol $\underline{38}(2)$ : 254-9. 
Greenwood AF, Powers RE und Jope RS (1995): Phosphoinositide hydrolysis, G alpha q, phospholipase $\mathrm{C}$, and protein kinase $\mathrm{C}$ in post mortem human brain: effects of post mortem interval, subject age, and Alzheimer's disease. Neuroscience 69(1): 125-38.

Grossberg GT (2002): The ABC of Alzheimer's disease: behavioral symptoms and their treatment. Int Psychogeriatr 14 Suppl 1: 27-49.

Growdon JH, Locascio JJ, Corkin S, Gomez-Isla T und Hyman BT (1996): Apolipoprotein E genotype does not influence rates of cognitive decline in Alzheimer's disease. Neurology 47(2): 444-8.

Grupe A, Abraham R, Li Y, Rowland C, Hollingworth P, Morgan A, Jehu L, Segurado R, Stone D, Schadt E, et al. (2007): Evidence for novel susceptibility genes for late-onset Alzheimer's disease from a genome-wide association study of putative functional variants. Hum Mol Genet 16(8): 865-73.

Haass C, Schlossmacher MG, Hung AY, Vigo-Pelfrey C, Mellon A, Ostaszewski BL, Lieberburg I, Koo EH, Schenk D, Teplow DB, et al. (1992): Amyloid beta-peptide is produced by cultured cells during normal metabolism. Nature 359(6393): 322-5.

Haass C, Hung AY, Selkoe DJ und Teplow DB (1994): Mutations associated with a locus for familial Alzheimer's disease result in alternative processing of amyloid beta-protein precursor. J Biol Chem 269(26): 17741-8.

Hardy J und Selkoe DJ (2002): The amyloid hypothesis of Alzheimer's disease: progress and problems on the road to therapeutics. Science 297(5580): 353-6.

Harold D, Abraham R, Hollingworth P, Sims R, Gerrish A, Hamshere ML, Pahwa JS, Moskvina V, Dowzell K, Williams A, et al. (2009): Genome-wide association study identifies variants at CLU and PICALM associated with Alzheimer's disease. Nat Genet 41(10): 1088-93.

Heinemann U, Krasnianski A, Meissner B, Varges D, Kallenberg K, Schulz-Schaeffer WJ, Steinhoff BJ, Grasbon-Frodl EM, Kretzschmar HA und Zerr I (2007): Creutzfeldt-Jakob disease in Germany: a prospective 12-year surveillance. Brain 130(Pt 5): 1350-9.

Herz J und Bock HH (2002): Lipoprotein receptors in the nervous system. Annu Rev Biochem $\underline{71}$ : 405-34. 
Hirono N, Hashimoto M, Yasuda M, Kazui H und Mori E (2003): Accelerated memory decline in Alzheimer's disease with apolipoprotein epsilon4 allele. J Neuropsychiatry Clin Neurosci 15(3): 354-8.

Hodges JR (2006): Alzheimer's centennial legacy: origins, landmarks and the current status of knowledge concerning cognitive aspects. Brain $\underline{129}(\mathrm{Pt} 11)$ : 2811-22.

Holland AJ, Hon J, Huppert FA, Stevens F und Watson P (1998): Population-based study of the prevalence and presentation of dementia in adults with Down's syndrome. Br J Psychiatry 172: 493-8.

Holmes C, Levy R, McLoughlin DM, Powell JF und Lovestone S (1996): Apolipoprotein E: noncognitive symptoms and cognitive decline in late onset Alzheimer's disease. J Neurol Neurosurg Psychiatry 61(6): 580-3.

Holmes C, Ballard C, Lehmann D, David Smith A, Beaumont H, Day IN, Nadeem Khan M, Lovestone S, McCulley M, Morris CM, et al. (2005): Rate of progression of cognitive decline in Alzheimer's disease: effect of butyrylcholinesterase K gene variation. J Neurol Neurosurg Psychiatry $\underline{76}$ (5): 640-3.

Honig LS und Mayeux R (2001): Natural history of Alzheimer's disease. Aging (Milano) 13(3): 17182.

Hoyt BD, Massman PJ, Schatschneider C, Cooke N und Doody RS (2005): Individual growth curve analysis of APOE epsilon 4-associated cognitive decline in Alzheimer disease. Arch Neurol 62(3): 454-9.

Hu J, Igarashi A, Kamata M und Nakagawa H (2001): Angiotensin-converting enzyme degrades Alzheimer amyloid beta-peptide (A beta ); retards A beta aggregation, deposition, fibril formation; and inhibits cytotoxicity. J Biol Chem 276(51): 47863-8.

Hu X, Pickering E, Liu YC, Hall S, Fournier H, Katz E, Dechairo B, John S, Van Eerdewegh P und Soares H (2011): Meta-analysis for genome-wide association study identifies multiple variants at the BIN1 locus associated with late-onset Alzheimer's disease. PLoS One $\underline{6}(2)$ : e16616.

Hui JS, Wilson RS, Bennett DA, Bienias JL, Gilley DW und Evans DA (2003): Rate of cognitive decline and mortality in Alzheimer's disease. Neurology 61(10): 1356-61. 
Hyman BT, West HL, Rebeck GW, Buldyrev SV, Mantegna RN, Ukleja M, Havlin S und Stanley HE (1995): Quantitative analysis of senile plaques in Alzheimer disease: observation of lognormal size distribution and molecular epidemiology of differences associated with apolipoprotein E genotype and trisomy 21 (Down syndrome). Proc Natl Acad Sci U S A 92(8): 3586-90.

Iqbal K, Alonso AD, Gondal JA, Gong CX, Haque N, Khatoon S, Sengupta A, Wang JZ und Grundke-Iqbal I (2000): Mechanism of neurofibrillary degeneration and pharmacologic therapeutic approach. J Neural Transm Suppl 59: 213-22.

Ito K, Ahadieh S, Corrigan B, French J, Fullerton T und Tensfeldt T (2010): Disease progression meta-analysis model in Alzheimer's disease. Alzheimers Dement 6 $\underline{6}(1): 39-53$.

Jack CR, Jr., Petersen RC, Xu YC, O'Brien PC, Smith GE, Ivnik RJ, Boeve BF, Waring SC, Tangalos EG und Kokmen E (1999): Prediction of AD with MRI-based hippocampal volume in mild cognitive impairment. Neurology 52(7): 1397-403.

Jack CR, Jr., Petersen RC, Xu Y, O'Brien PC, Smith GE, Ivnik RJ, Boeve BF, Tangalos EG und Kokmen E (2000): Rates of hippocampal atrophy correlate with change in clinical status in aging and AD. Neurology 55(4): 484-89.

Jagust W (2006): Positron emission tomography and magnetic resonance imaging in the diagnosis and prediction of dementia. Alzheimers Dement 2(1): 36-42.

Jayaratnam S, Khoo AK und Basic D (2008): Rapidly progressive Alzheimer's disease and elevated 14-3-3 proteins in cerebrospinal fluid. Age Ageing 37(4): 467-9.

Jellinger KA (2004): Head injury and dementia. Curr Opin Neurol 17(6): 719-23.

Jonker C, Schmand B, Lindeboom J, Havekes LM und Launer LJ (1998): Association between apolipoprotein E epsilon4 and the rate of cognitive decline in community-dwelling elderly individuals with and without dementia. Arch Neurol 55(8): 1065-9.

Jope RS, Song L, Li X und Powers R (1994): Impaired phosphoinositide hydrolysis in Alzheimer's disease brain. Neurobiol Aging 15(2): 221-6. 
Josephs KA, Ahlskog JE, Parisi JE, Boeve BF, Crum BA, Giannini C und Petersen RC (2009):

Rapidly progressive neurodegenerative dementias. Arch Neurol 66 (2): 201-7.

Jost BC und Grossberg GT (1995): The natural history of Alzheimer's disease: a brain bank study. J Am Geriatr Soc 43(11): 1248-55.

Kalvodova L, Kahya N, Schwille P, Ehehalt R, Verkade P, Drechsel D und Simons K (2005): Lipids as modulators of proteolytic activity of BACE: involvement of cholesterol, glycosphingolipids, and anionic phospholipids in vitro. J Biol Chem 280(44): 36815-23.

Kamboh MI, Minster RL, Kenney M, Ozturk A, Desai PP, Kammerer CM und DeKosky ST (2006): Alpha-1-antichymotrypsin (ACT or SERPINA3) polymorphism may affect age-at-onset and disease duration of Alzheimer's disease. Neurobiol Aging 27(10): 1435-9.

Kanai M, Shizuka M, Urakami K, Matsubara E, Harigaya Y, Okamoto K und Shoji M (1999): Apolipoprotein E4 accelerates dementia and increases cerebrospinal fluid tau levels in Alzheimer's disease. Neurosci Lett 267(1): 65-8.

Kester MI, van der Vlies AE, Blankenstein MA, Pijnenburg YA, van Elk EJ, Scheltens P und van der Flier WM (2009): CSF biomarkers predict rate of cognitive decline in Alzheimer disease. Neurology 73(17): 1353-8.

Kim J, Basak JM und Holtzman DM (2009): The role of apolipoprotein E in Alzheimer's disease. Neuron $\underline{63}(3)$ : 287-303.

Kleiman T, Zdanys K, Black B, Rightmer T, Grey M, Garman K, Macavoy M, Gelernter J und van Dyck C (2006): Apolipoprotein E epsilon4 allele is unrelated to cognitive or functional decline in Alzheimer's disease: retrospective and prospective analysis. Dement Geriatr Cogn Disord 22(1): 73-82.

Kurz A, Egensperger R, Haupt M, Lautenschlager N, Romero B, Graeber MB und Muller U (1996): Apolipoprotein E epsilon 4 allele, cognitive decline, and deterioration of everyday performance in Alzheimer's disease. Neurology 47(2): 440-3.

Lambert JC und Amouyel P (2011): Genetics of Alzheimer's disease: new evidences for an old hypothesis? Curr Opin Genet Dev 21(3): 295-301. 
Lawton MP und Brody EM (1969): Assessment of older people: self-maintaining and instrumental activities of daily living. Gerontologist 9 (3): 179-86.

Lehericy S, Marjanska M, Mesrob L, Sarazin M und Kinkingnehun S (2007): Magnetic resonance imaging of Alzheimer's disease. Eur Radiol 17(2): 347-62.

Lehtovirta M, Kuikka J, Helisalmi S, Hartikainen P, Mannermaa A, Ryynanen M, Riekkinen P und Soininen H (1998): Longitudinal SPECT study in Alzheimer's disease: relation to apolipoprotein E polymorphism. J Neurol Neurosurg Psychiatry 64(6): 742-6.

Lemere CA, Blusztajn JK, Yamaguchi H, Wisniewski T, Saido TC und Selkoe DJ (1996): Sequence of deposition of heterogeneous amyloid beta-peptides and APO E in Down syndrome: implications for initial events in amyloid plaque formation. Neurobiol Dis $\underline{3}(1)$ : 16-32.

Levy-Lahad E, Wasco W, Poorkaj P, Romano DM, Oshima J, Pettingell WH, Yu CE, Jondro PD, Schmidt SD, Wang K, et al. (1995): Candidate gene for the chromosome 1 familial Alzheimer's disease locus. Science 269(5226): 973-7.

Li K, Dai D, Zhao B, Yao L, Yao S, Wang B und Yang Z (2010): Association between the RAGE G82S polymorphism and Alzheimer's disease. J Neural Transm 117(1): 97-104.

Licastro F, Chiappelli M, Grimaldi LM, Morgan K, Kalsheker N, Calabrese E, Ritchie A, Porcellini E, Salani G, Franceschi M, et al. (2005): A new promoter polymorphism in the alpha-1antichymotrypsin gene is a disease modifier of Alzheimer's disease. Neurobiol Aging 26(4): 449-53.

Lobo A, Launer LJ, Fratiglioni L, Andersen K, Di Carlo A, Breteler MM, Copeland JR, Dartigues JF, Jagger C, Martinez-Lage J, et al. (2000): Prevalence of dementia and major subtypes in Europe: A collaborative study of population-based cohorts. Neurologic Diseases in the Elderly Research Group. Neurology 54(11 Suppl 5): S4-9.

Luchsinger JA und Mayeux R (2004): Dietary factors and Alzheimer's disease. Lancet Neurol $\underline{3}(10)$ : 579-87.

Mahlberg R und Gutzmann H (2005): Zertifizierte medizinische Fortbildung: Diagnostik von Demenzerkrankungen. Dtsch Ärztebl 102(28-29): A2032-39. 
Mann UM, Mohr E und Chase TN (1989): Rapidly progressive Alzheimer's disease. Lancet 1989, 2: 799.

Marra C, Silveri MC und Gainotti G (2000): Predictors of cognitive decline in the early stage of probable Alzheimer's disease. Dement Geriatr Cogn Disord 11(4): 212-8.

Marra C, Bizzarro A, Daniele A, De Luca L, Ferraccioli M, Valenza A, Brahe C, Tiziano FD, Gainotti G und Masullo C (2004): Apolipoprotein E epsilon4 allele differently affects the patterns of neuropsychological presentation in early- and late-onset Alzheimer's disease patients. Dement Geriatr Cogn Disord 18(2): 125-31.

Martins CA, Oulhaj A, de Jager CA und Williams JH (2005): APOE alleles predict the rate of cognitive decline in Alzheimer disease: a nonlinear model. Neurology 65(12): 1888-93.

Masters CL, Simms G, Weinman NA, Multhaup G, McDonald BL und Beyreuther K (1985): Amyloid plaque core protein in Alzheimer disease and Down syndrome. Proc Natl Acad Sci U S A 82(12): 4245-9.

Mawuenyega KG, Sigurdson W, Ovod V, Munsell L, Kasten T, Morris JC, Yarasheski KE und Bateman RJ (2010): Decreased clearance of CNS beta-amyloid in Alzheimer's disease. Science 330(6012): 1774.

Mayeux R: Alzheimer's disease: epidemiology. In: Handbook of Clinical Neurology. Duyckaerts C und Litvan I (Hrsg.); Vol. 89; Elsevier, Amsterdam 2008, 195-205.

McKeith I und Cummings J (2005): Behavioural changes and psychological symptoms in dementia disorders. Lancet Neurol 4(11): 735-42.

McKhann G, Drachman D, Folstein M, Katzman R, Price D und Stadlan EM (1984): Clinical diagnosis of Alzheimer's disease: report of the NINCDS-ADRDA Work Group under the auspices of Department of Health and Human Services Task Force on Alzheimer's Disease. Neurology $\underline{34}$ (7): 939-44.

Mielke MM, Rosenberg PB, Tschanz J, Cook L, Corcoran C, Hayden KM, Norton M, Rabins PV, Green RC, Welsh-Bohmer KA, et al. (2007): Vascular factors predict rate of progression in Alzheimer disease. Neurology 69(19): 1850-8. 
Mori E, Lee K, Yasuda M, Hashimoto M, Kazui H, Hirono N und Matsui M (2002): Accelerated hippocampal atrophy in Alzheimer's disease with apolipoprotein E epsilon4 allele. Ann Neurol 51(2): 209-14.

Morris JC, Mohs RC, Rogers H, Fillenbaum G und Heyman A (1988): Consortium to establish a registry for Alzheimer's disease (CERAD) clinical and neuropsychological assessment of Alzheimer's disease. Psychopharmacol Bull 24(4): 641-52.

Morris JC, Heyman A, Mohs RC, Hughes JP, van Belle G, Fillenbaum G, Mellits ED und Clark C (1989): The Consortium to Establish a Registry for Alzheimer's Disease (CERAD). Part I. Clinical and neuropsychological assessment of Alzheimer's disease. Neurology 39(9): 115965 .

Morris JC, Edland S, Clark C, Galasko D, Koss E, Mohs R, van Belle G, Fillenbaum G und Heyman A (1993): The consortium to establish a registry for Alzheimer's disease (CERAD). Part IV. Rates of cognitive change in the longitudinal assessment of probable Alzheimer's disease. Neurology $\underline{43}$ (12): 2457-65.

Murphy GM, Jr., Taylor J, Kraemer HC, Yesavage J und Tinklenberg JR (1997): No association between apolipoprotein E epsilon 4 allele and rate of decline in Alzheimer's disease. Am J Psychiatry 154(5): 603-8.

Murphy GM, Jr., Claassen JD, DeVoss JJ, Pascoe N, Taylor J, Tinklenberg JR und Yesavage JA (2001): Rate of cognitive decline in AD is accelerated by the interleukin-1 alpha $-889 * 1$ allele. Neurology $\underline{56}(11):$ 1595-7.

Murray ME, Graff-Radford NR, Ross OA, Petersen RC, Duara R und Dickson DW (2011): Neuropathologically defined subtypes of Alzheimer's disease with distinct clinical characteristics: a retrospective study. Lancet Neurol 10(9): 785-96.

Newkirk LA, Kim JM, Thompson JM, Tinklenberg JR, Yesavage JA und Taylor JL (2004): Validation of a 26-point telephone version of the Mini-Mental State Examination. J Geriatr Psychiatry Neurol 17(2): 81-7.

NICE-SCIE: Dementia. A NICE-SCIE Guideline on supporting people with dementia and their carers in health and social care; The British Psychological Society and Gaskell, London 2007. 
O'Brien KK, Saxby BK, Ballard CG, Grace J, Harrington F, Ford GA, O'Brien JT, Swan AG, Fairbairn AF, Wesnes K, et al. (2003): Regulation of attention and response to therapy in dementia by butyrylcholinesterase. Pharmacogenetics 13(4): 231-9.

Petersen RC und Negash S (2008): Mild cognitive impairment: an overview. CNS Spectr 13(1): 45-53.

Poirier J, Davignon J, Bouthillier D, Kogan S, Bertrand P und Gauthier S (1993): Apolipoprotein E polymorphism and Alzheimer's disease. Lancet $\underline{342(8873): ~ 697-9 . ~}$

Porcellini E, Calabrese E, Guerini F, Govoni M, Chiappelli M, Tumini E, Morgan K, Chappell S, Kalsheker N, Franceschi M, et al. (2007): The hydroxy-methyl-glutaryl CoA reductase promoter polymorphism is associated with Alzheimer's risk and cognitive deterioration. Neurosci Lett 416(1): 66-70.

Portet F, Scarmeas N, Cosentino S, Helzner EP und Stern Y (2009): Extrapyramidal signs before and after diagnosis of incident Alzheimer disease in a prospective population study. Arch Neurol 66(9): 1120-6.

Potkin SG (2002): The ABC of Alzheimer's disease: ADL and improving day-to-day functioning of patients. Int Psychogeriatr 14 Suppl 1: 7-26.

Qiu C, Kivipelto M und von Strauss E (2009): Epidemiology of Alzheimer's disease: occurrence, determinants, and strategies toward intervention. Dialogues Clin Neurosci 11(2): 111-28.

Querfurth HW und LaFerla FM (2010): Alzheimer's disease. N Engl J Med 362(4): 329-44.

Rebeck GW, Reiter JS, Strickland DK und Hyman BT (1993): Apolipoprotein E in sporadic Alzheimer's disease: allelic variation and receptor interactions. Neuron 11(4): 575-80.

Refolo LM, Malester B, LaFrancois J, Bryant-Thomas T, Wang R, Tint GS, Sambamurti K, Duff K und Pappolla MA (2000): Hypercholesterolemia accelerates the Alzheimer's amyloid pathology in a transgenic mouse model. Neurobiol Dis $\underline{7}(4): 321-31$.

Reinwald S, Westner IM und Niedermaier N (2004): Rapidly progressive Alzheimer's disease mimicking Creutzfeldt-Jakob disease. J Neurol 251(8): 1020-2. 
Reisberg B, Ferris SH, de Leon MJ und Crook T (1988): Global Deterioration Scale (GDS). Psychopharmacol Bull 24(4): 661-3.

Roselli F, Tartaglione B, Federico F, Lepore V, Defazio G und Livrea P (2009): Rate of MMSE score change in Alzheimer's disease: influence of education and vascular risk factors. Clin Neurol Neurosurg 111(4): 327-30.

Rusinek H, Endo Y, De Santi S, Frid D, Tsui WH, Segal S, Convit A und de Leon MJ (2004): Atrophy rate in medial temporal lobe during progression of Alzheimer disease. Neurology 63(12): 2354-9.

Sastre M, Calero M, Pawlik M, Mathews PM, Kumar A, Danilov V, Schmidt SD, Nixon RA, Frangione B und Levy E (2004): Binding of cystatin C to Alzheimer's amyloid beta inhibits in vitro amyloid fibril formation. Neurobiol Aging 25(8): 1033-43.

Scarmeas N, Hadjigeorgiou GM, Papadimitriou A, Dubois B, Sarazin M, Brandt J, Albert M, Marder K, Bell K, Honig LS, et al. (2004): Motor signs during the course of Alzheimer disease. Neurology 63(6): 975-82.

Schaub RT und Freyberger HJ: Diagnostik und Klassifikation von Demenzen. In: Demenzen. Wallesch CW und Förstl H (Hrsg.); Georg Thieme Verlag, Stuttgart 2005, 59-81.

Scheltens P (2009): Imaging in Alzheimer's disease. Dialogues Clin Neurosci 11(2): 191-9.

Schmechel DE, Saunders AM, Strittmatter WJ, Crain BJ, Hulette CM, Joo SH, Pericak-Vance MA, Goldgaber D und Roses AD (1993): Increased amyloid beta-peptide deposition in cerebral cortex as a consequence of apolipoprotein E genotype in late-onset Alzheimer disease. Proc Natl Acad Sci U S A 90(20): 9649-53.

Schmidt C, Redyk K, Meissner B, Krack L, von Ahsen N, Roeber S, Kretzschmar H und Zerr I (2010): Clinical features of rapidly progressive Alzheimer's disease. Dement Geriatr Cogn Disord 29(4): 371-8.

Schmidt C, Wolff M, Weitz M, Bartlau T, Korth C und Zerr I (2011): Rapidly progressive Alzheimer disease. Arch Neurol $\underline{68}(9): 1124-30$. 
Schmidtke K und Hüll M: Alzheimer-Demenz. In: Demenzen. Wallesch CW und Förstl H (Hrsg.); Georg Thieme Verlag, Stuttgart 2005, 152-175.

Schütz E und von Ahsen N (1999): Spreadsheet software for thermodynamic melting point prediction of oligonucleotide hybridization with and without mismatches. Biotechniques 27(6): 1218-22, 1224.

Selkoe DJ: Biochemistry and Molecular Biology of Amyloid beta-Protein and the Mechanism of Alzheimer's Disease. In: Handbook of Clinical Neurology. Duyckaerts C und Litvan I (Hrsg.); Vol. 89; Elsevier, Amsterdam 2008, 245-60.

Sherrington R, Rogaev EI, Liang Y, Rogaeva EA, Levesque G, Ikeda M, Chi H, Lin C, Li G, Holman $\mathrm{K}$, et al. (1995): Cloning of a gene bearing missense mutations in early-onset familial Alzheimer's disease. Nature 375(6534): 754-60.

Sherrington R, Froelich S, Sorbi S, Campion D, Chi H, Rogaeva EA, Levesque G, Rogaev EI, Lin C, Liang Y, et al. (1996): Alzheimer's disease associated with mutations in presenilin 2 is rare and variably penetrant. Hum Mol Genet $\underline{5}(7)$ : 985-8.

Sleegers K, Lambert JC, Bertram L, Cruts M, Amouyel P und Van Broeckhoven C (2009): The pursuit of susceptibility genes for Alzheimer's disease: progress and prospects. Trends Genet 26(2): 84-93.

Slooter AJ, Houwing-Duistermaat JJ, van Harskamp F, Cruts M, Van Broeckhoven C, Breteler MM, Hofman A, Stijnen T und van Duijn CM (1999): Apolipoprotein E genotype and progression of Alzheimer's disease: the Rotterdam Study. J Neurol 246(4): 304-8.

Smith MZ, Esiri MM, Barnetson L, King E und Nagy Z (2001): Constructional apraxia in Alzheimer's disease: association with occipital lobe pathology and accelerated cognitive decline. Dement Geriatr Cogn Disord 12(4): 281-8.

Snider BJ, Fagan AM, Roe C, Shah AR, Grant EA, Xiong C, Morris JC und Holtzman DM (2009): Cerebrospinal fluid biomarkers and rate of cognitive decline in very mild dementia of the Alzheimer type. Arch Neurol 66(5): 638-45. 
Soto ME, Andrieu S, Cantet C, Reynish E, Ousset PJ, Arbus C, Gillette-Guyonnet S, Nourhashemi F und Vellas B (2008a): Predictive value of rapid decline in mini mental state examination in clinical practice for prognosis in Alzheimer's disease. Dement Geriatr Cogn Disord 26(2): 109-16.

Soto ME, Andrieu S, Arbus C, Ceccaldi M, Couratier P, Dantoine T, Dartigues JF, Gillette-Guyonnet S, Nourhashemi F, Ousset PJ, et al. (2008b): Rapid cognitive decline in Alzheimer's disease. Consensus paper. J Nutr Health Aging 12(10): 703-13.

Soubelet A und Salthouse TA (2011): Correlates of level and change in the mini-mental state examination. Psychol Assess 23(4): 811-8.

Starkstein SE, Jorge R, Mizrahi R und Robinson RG (2006): A prospective longitudinal study of apathy in Alzheimer's disease. J Neurol Neurosurg Psychiatry $\underline{77(1): 8-11 .}$

Stern Y, Andrews H, Pittman J, Sano M, Tatemichi T, Lantigua R und Mayeux R (1992): Diagnosis of dementia in a heterogeneous population. Development of a neuropsychological paradigmbased diagnosis of dementia and quantified correction for the effects of education. Arch Neurol 49(5): 453-60.

Stern Y, Brandt J, Albert M, Jacobs DM, Liu X, Bell K, Marder K, Sano M, Albert S, Del-Castillo Castenada C, et al. (1997): The absence of an apolipoprotein epsilon4 allele is associated with a more aggressive form of Alzheimer's disease. Ann Neurol 41(5): 615-20.

Stopa EG, Volicer L, Kuo-Leblanc V, Harper D, Lathi D, Tate B und Satlin A (1999): Pathologic evaluation of the human suprachiasmatic nucleus in severe dementia. J Neuropathol Exp Neurol $\underline{58}(1): 29-39$.

Strittmatter WJ, Saunders AM, Schmechel D, Pericak-Vance M, Enghild J, Salvesen GS und Roses AD (1993): Apolipoprotein E: high-avidity binding to beta-amyloid and increased frequency of type 4 allele in late-onset familial Alzheimer disease. Proc Natl Acad Sci U S A 90(5): 1977-81.

Tanzi RE (1999): A genetic dichotomy model for the inheritance of Alzheimer's disease and common age-related disorders. J Clin Invest 104(9): 1175-9. 
Terry AV, Jr. und Buccafusco JJ (2003): The cholinergic hypothesis of age and Alzheimer's diseaserelated cognitive deficits: recent challenges and their implications for novel drug development. J Pharmacol Exp Ther 306(3): 821-7.

Thalmann B, Monsch AU, Schneitter M, Bernasconi F, Aebi C, Camachova-Davet Z und Staehelin HB (2000): The CERAD neuropsychological assessment battery (CERAD-NAB) - A minimal dataset as a common tool for German-speaking Europe. Neurobiol Aging 21 Suppl.1: 30.

Tschampa HJ, Neumann M, Zerr I, Henkel K, Schroter A, Schulz-Schaeffer WJ, Steinhoff BJ, Kretzschmar HA und Poser S (2001): Patients with Alzheimer's disease and dementia with

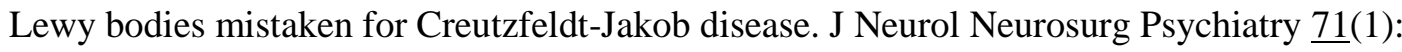
33-9.

Tschanz JT, Corcoran CD, Schwartz S, Treiber K, Green RC, Norton MC, Mielke MM, Piercy K, Steinberg M, Rabins PV, et al. (2011): Progression of cognitive, functional, and neuropsychiatric symptom domains in a population cohort with Alzheimer dementia: the Cache County Dementia Progression study. Am J Geriatr Psychiatry 19(6): 532-42.

Tumini E, Porcellini E, Chiappelli M, Conti CM, Beraudi A, Poli A, Caciagli F, Doyle R, Conti P und Licastro F (2007): The G51S purine nucleoside phosphorylase polymorphism is associated with cognitive decline in Alzheimer's disease patients. Hum Psychopharmacol 22(2): 75-80.

van der Vlies AE, Koedam EL, Pijnenburg YA, Twisk JW, Scheltens P und van der Flier WM (2009): Most rapid cognitive decline in APOE epsilon4 negative Alzheimer's disease with early onset. Psychol Med 39(11): 1907-11.

Van Everbroeck B, Dobbeleir I, De Waele M, De Deyn P, Martin JJ und Cras P (2004): Differential diagnosis of 201 possible Creutzfeldt-Jakob disease patients. J Neurol 251(3): 298-304.

Vardy ER, Rice PJ, Bowie PC, Holmes JD, Catto AJ und Hooper NM (2009): Plasma angiotensinconverting enzyme in Alzheimer's disease. J Alzheimers Dis 16(3): 609-18.

Wallin AK, Blennow K, Andreasen N und Minthon L (2006): CSF biomarkers for Alzheimer's Disease: levels of beta-amyloid, tau, phosphorylated tau relate to clinical symptoms and survival. Dement Geriatr Cogn Disord 21(3): 131-8. 
Wallin AK, Blennow K, Zetterberg H, Londos E, Minthon L und Hansson O (2010): CSF biomarkers predict a more malignant outcome in Alzheimer disease. Neurology 74(19): 1531-7.

Wechsler-Reya R, Sakamuro D, Zhang J, Duhadaway J und Prendergast GC (1997): Structural analysis of the human BIN1 gene. Evidence for tissue-specific transcriptional regulation and alternate RNA splicing. J Biol Chem 272(50): 31453-8.

WHO, Dilling H, Mombour W und Schmidt MH: Internationale Klassifikation psychischer Störungen: ICD-10 Kapitel V (F). Klinisch-diagnostische Leitlinien; Huber, Bern 2011a.

WHO, Dilling H, Mombour W, Schmidt MH und Schulte-Markwort E: Internationale Klassifikation psychischer Störungen. ICD-10 Kapitel V (F). Diagnostische Kriterien für Forschung und Praxis; Huber, Bern 2011b.

Wigge P und McMahon HT (1998): The amphiphysin family of proteins and their role in endocytosis at the synapse. Trends Neurosci 21(8): 339-44.

Wigge P, Kohler K, Vallis Y, Doyle CA, Owen D, Hunt SP und McMahon HT (1997): Amphiphysin heterodimers: potential role in clathrin-mediated endocytosis. Mol Biol Cell $\underline{8}(10):$ 2003-15.

Wilcock GK (2003): Memantine for the treatment of dementia. Lancet Neurol 2(8): 503-5.

Wilkosz PA, Seltman HJ, Devlin B, Weamer EA, Lopez OL, DeKosky ST und Sweet RA (2010): Trajectories of cognitive decline in Alzheimer's disease. Int Psychogeriatr 22(2): 281-90.

Wimo A, Winblad B und Jonsson L (2010): The worldwide societal costs of dementia: Estimates for 2009. Alzheimers Dement $\underline{6}(2)$ : 98-103.

Wolf BA, Wertkin AM, Jolly YC, Yasuda RP, Wolfe BB, Konrad RJ, Manning D, Ravi S, Williamson JR und Lee VM (1995): Muscarinic regulation of Alzheimer's disease amyloid precursor protein secretion and amyloid beta-protein production in human neuronal NT2N cells. J Biol Chem 270(9): 4916-22.

www.destatis.de: Statistisches Bundesamt, Pressemitteilung Nr.280 vom 11.08.2010: Hohe Kosten durch Demenz und Depressionen, im August 2010 
Yaffe K, Lindquist K, Shlipak MG, Simonsick E, Fried L, Rosano C, Satterfield S, Atkinson H, Windham BG und Kurella-Tamura M (2008): Cystatin C as a marker of cognitive function in elders: findings from the health ABC study. Ann Neurol 63(6): 798-802.

Zerr I und Poser S (2002): Clinical diagnosis and differential diagnosis of CJD and vCJD. With special emphasis on laboratory tests. APMIS $\underline{110}(1)$ : 88-98.

Zetterberg H und Blennow K: Biological CSF Markers of Alzheimer's Disease. In: Handbook of Clinical Neurology. Duyckaerts C und Litvan I (Hrsg.); Vol. 89; Elsevier, Amsterdam 2008, 261-8.

Ziegler U und Doblhammer G (2009): Prevalence and incidence of dementia in Germany - a study based on data from the public sick funds in 2002. Gesundheitswesen 71(5): 281-90. 


\section{Publikationen}

Teile dieser Arbeit wurden bereits im Rahmen folgender Publikationen veröffentlicht:

Schmidt C, Wolff M, Weitz M, Bartlau T, Korth C und Zerr I (2011): Rapidly progressive Alzheimer disease. Arch Neurol 68(9): 1124-30.

Schmidt C, Wolff M, Shalash A and Zerr I: Risk Factors for Disease Progression in Alzheimer's Disease. In: The Clinical Spectrum of Alzheimer's Disease -The Charge Toward Comprehensive Diagnostic and Therapeutic Strategies; Suzanne De La Monte (Hrsg.); InTech, 2011, 3-20.

Schmidt C, Wolff M, von Ahsen N und Zerr I (2012): Alzheimer's disease: genetic polymorphisms and rate of decline. Dement Geriatr Cogn Disord 33(2-3): 84-9. 


\section{Danksagung}

Mein herzlicher Dank gilt in besonderem Maße Frau Prof. Dr. med. Inga Zerr (Abteilung Neurologie, Universitätsmedizin Göttingen) für die Möglichkeit, das interessante Thema im Rahmen der Alzheimer-Studie bearbeiten zu können sowie für die wertvollen Ratschläge und Anregungen während der Anfertigung dieser Arbeit.

Zudem möchte ich mich ganz herzlich bei Herrn Dr. med. Christian Schmidt (ebenfalls Abteilung Neurologie, Universitätsmedizin Göttingen) für die hervorragende Betreuung und die fachliche und moralische Unterstützung während aller Phasen der Dissertation bedanken.

Mein besonderer Dank gilt auch Herrn Prof. Dr. med. Nicolas von Ahsen, ehemals Abteilung für Klinische Chemie der Universitätsmedizin Göttingen, für die engagierte Hilfestellung bei den genetischen und statistischen Fragestellungen sowie den Mitarbeiterinnen und Mitarbeitern dieser Abteilung für die aufwändige Bestimmung der genetischen Polymorphismen.

Des Weiteren möchte ich mich bei den Mitarbeiterinnen und Mitarbeitern des Prion-Labors für die Aufbereitung der Blut- und Liquorproben sowie die Bestimmung der Demenzmarker bedanken.

Außerdem danke ich allen Ärztinnen und Ärzten sowie Mitarbeiterinnen und Mitarbeitern der Prionforschungsgruppe für die gute Zusammenarbeit und eine sehr schöne Zeit. 


\section{Lebenslauf}

Am 17.02.1981 wurde ich als drittes Kind von Dr. med. Elke Wolff und Dr. med. Reinhard Wolff in Braunschweig geboren. Hier wuchs ich auch auf und absolvierte meine Schulausbildung, welche ich im Juni 2000 am Wilhelm-Gymnasium mit dem Abitur abschloss.

Von August 2000 bis Juli 2001 leistete ich meinen Zivildienst bei der Johanniter Unfallhilfe in Braunschweig. Es folgte vom Oktober 2001 bis zum Februar 2002 für ein Semester das Studium der Medizininformatik in Lübeck.

Im Frühjahr 2002 nahm ich schließlich das Studium der Humanmedizin an der Georg-AugustUniversität in Göttingen auf, welches ich im Mai 2009 mit dem Staatsexamen erfolgreich abschloss. Nach Erlangung der Ärztlichen Approbation begann ich im Juni 2009 meine Dissertation unter der Leitung von Frau Prof. Dr. Inga Zerr in der Abteilung für Neurologie der Universitätsmedizin Göttingen. In diesem Rahmen betreute ich als Stipendiat bis September 2010 die der Dissertation zugrundeliegende klinische Studie zum Thema „rapidprogressive Alzheimer-Demenz“"

Von November 2010 bis Februar 2011 absolvierte ich beim Georg Thieme Verlag in Stuttgart ein Praktikum in der Redaktion der Fachzeitschrift Via medici, für die ich seitdem als freier Mitarbeiter tätig bin.

Im April 2011 trat ich eine Stelle als Assistenzarzt in der Neurologischen Abteilung der Universitätsmedizin Göttingen an. Seit Oktober 2011 bin ich als Assistenzarzt in der Klinik für Neurologie und Neurophysiologie des St. Bernward-Krankenhauses in Hildesheim beschäftigt. 